\title{
Ambient Monitoring for Sinclair and Dyes Inlets, Puget Sound, Washington: Chemical Analyses for 2010 Regional Mussel Watch (AMB02)
}

J. M. Brandenberger

L. J. Kuo

C.R. Suslick

Pacific Northwest National Marine Sciences

Laboratory, Sequim, Washington

R. K. Johnston

Space and Naval Warfare Systems Center Bremerton, Washington

September 2010

Prepared for the

Puget Sound Naval Shipyard and

Intermediate Maintenance Facility

Project ENVVEST

Bremerton, Washington

under Contract MIPR \# N4523A10MP00034

Pacific Northwest National Laboratory

Richland, Washington

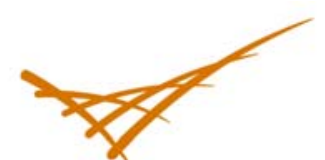

Pacific Northwest 


\title{
DISCLAIMER
}

This report was prepared as an account of work sponsored by an agency of the United States Government. Neither the United States Government nor any agency thereof, nor Battelle Memorial Institute, nor any of their employees, makes any warranty, express or implied, or assumes any legal liability or responsibility for the accuracy, completeness, or usefulness of any information, apparatus, product, or process disclosed, or represents that its use would not infringe privately owned rights. Reference herein to any specific commercial product, process, or service by trade name, trademark, manufacturer, or otherwise does not necessarily constitute or imply its endorsement, recommendation, or favoring by the United States Government or any agency thereof, or Battelle Memorial Institute. The views and opinions of authors expressed herein do not necessarily state or reflect those of the United States Government or any agency thereof.

\author{
PACIFIC NORTHWEST NATIONAL LABORATORY \\ operated by \\ BATTELLE MEMORIAL INSTITUTE \\ for the \\ UNITED STATES DEPARTMENT OF ENERGY \\ under Contract DE-AC06-76RLO 1830 \\ Printed in the United States of America \\ Available to DOE and DOE contractors from the \\ Office of Scientific and Technical Information, \\ P.O. Box 62, Oak Ridge, TN 37831; \\ prices available from (615) 576-8401.
}

Available to the public from the National Technical Information Service,

U.S. Department of Commerce,

5285 Port Royal Rd., Springfield, VA 22161

This document was printed on recycled paper.

PNNL-19845 
Ambient Monitoring for Sinclair and Dyes Inlets, Washington: Chemical Analyses for 2010 Regional Mussel Watch (AMB02)

J. M. Brandenberger

L. J. Kuo

C.R. Suslick

Pacific Northwest National Marine Sciences Laboratory

Sequim, Washington

R. K. Johnston

Space and Naval Warfare Systems Center

Bremerton, Washington

September 2010

Prepared for

Puget Sound Naval Shipyard and

Intermediate Maintenance Facility

Project ENVVEST

Bremerton, Washington

under Contract MIPR \# N4523A10MP00034

Pacific Northwest National Laboratory

Richland, Washington 99352 


\begin{abstract}
The Puget Sound Naval Shipyard \& Intermediate Maintenance Facility (PSNS\&IMF) and Naval Base Kitsap-Bremerton (Shipyard) located in Bremerton, WA are committed to a culture of continuous process improvement for all aspects of Shipyard operations, including reducing the releases of hazardous materials and waste in discharges from the Shipyard. Under the Project ENVVEST Final Project Agreement, a cooperative project among PSNS\&IMF, the Environmental Protection Agency (EPA), and the Washington State Department of Ecology (Ecology), and local stakeholders (US Navy, EPA and Ecology 2002) has been helping to improve the environmental quality of the Sinclair and Dyes Inlet Watershed (ENVVEST 2006). An ambient monitoring program for sediment, water, and indigenous mussels began in 2009 to assess the status and trend of ecological resources, assess the effectiveness of cleanup and pollution control measures, and determine if discharges from all sources are protective of beneficial uses including aquatic life. This document presents the 2010 chemical residue data and stable isotopes of carbon $\left(\delta^{13} \mathrm{C}\right)$ and nitrogen $\left(\delta^{15} \mathrm{~N}\right)$ for the regional mussel watch stations located in Sinclair Inlet, Dyes Inlet, Port Orchard Passage, Rich Passage, Agate Passage, Liberty Bay, and Keyport Lagoon. Indigenous bivalves were collected from a small boat and/or from along the shoreline, measured, composited, and analyzed for a suite of trace metals and organic contaminants. The trace metals included silver, arsenic, cadmium, chromium, copper, mercury, nickel, lead, and zinc. The organic contaminants included the list of NOAA Status and Trends 20 polychlorinated biphenyls (PCB) congeners and suite of parent and methylated polycyclic aromatic hydrocarbons (PAHs). These chemical residue data provide the first year of the biota ambient monitoring.
\end{abstract}




\section{$\underline{\text { Table of Content }}$}

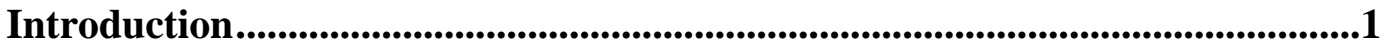

Sample Collection and Analysis....................................................................................2

Field Data Summary .......................................................................................................7

Metals Results, Wet Weight Basis .................................................................

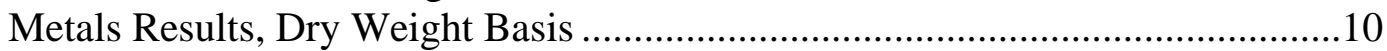

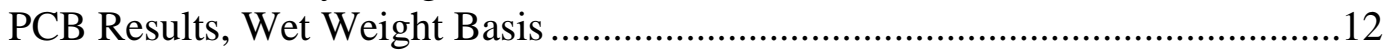

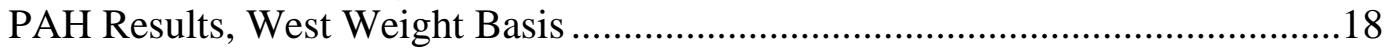

Stable Isotopes, Dry Weight Basis....................................................................30

QA/QC Sample Results .............................................................................31

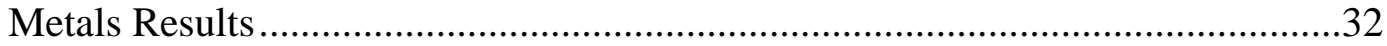

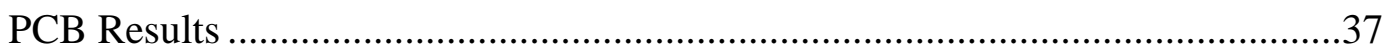

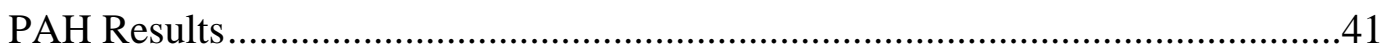

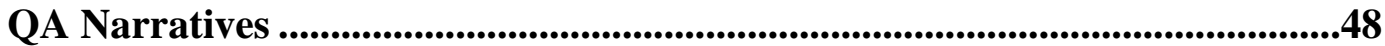

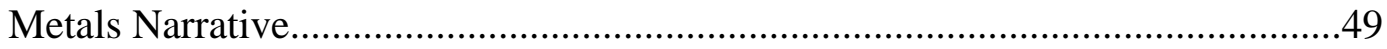

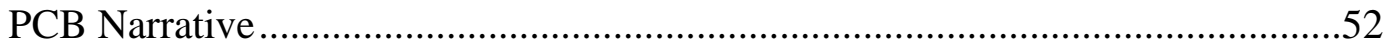

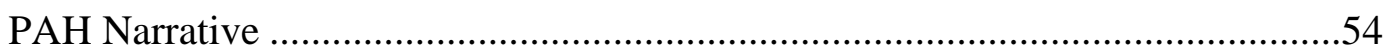

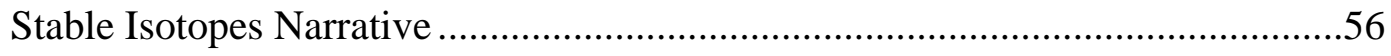

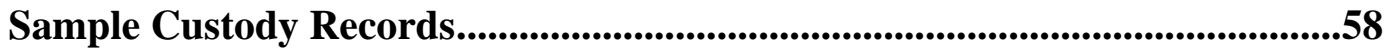

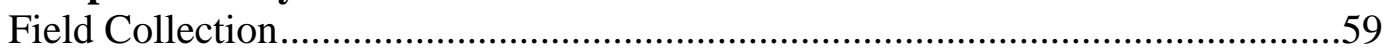

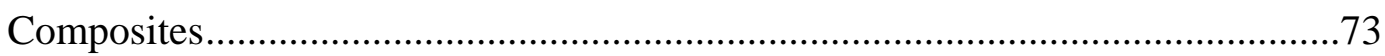

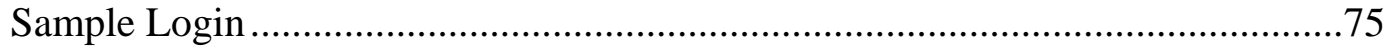

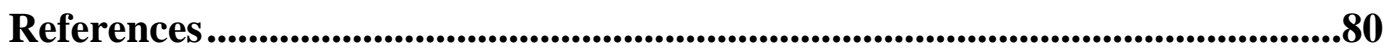




\section{Introduction}

Sinclair Inlet and Dyes Inlet were listed on the State of Washington's 1998 Section 303(d) list of impaired waters because of fecal coliform contamination in marine waters and tributary streams, heavy metals and toxic organics in the bottom sediments, and polycyclic aromatic hydrocarbons (PAHs), polychlorinated biphenyl (PCBs), aldrin, dieldrin, mercury $(\mathrm{Hg})$, and arsenic (As) in the tissues of marine organisms. The Puget Sound Naval Shipyard \& Intermediate Maintenance Facility (PSNS\&IMF) and Naval Base Kitsap-Bremerton (referred to as Shipyard) located in Bremerton, WA are committed to a culture of continuous process improvement for all aspects of Shipyard operations, including reducing the releases of hazardous materials and waste in discharges from the Shipyard. Under the Project ENVVEST Final Project Agreement, a cooperative project among PSNS\&IMF, the Environmental Protection Agency (EPA), and the Washington State Department of Ecology (Ecology), and local stakeholders (US Navy, EPA and Ecology 2002) has been helping to improve the environmental quality of the Sinclair and Dyes Inlet Watershed (ENVVEST 2006). An ambient monitoring program for sediment, water, and indigenous mussels began in 2009 to assess the status and trend of ecological resources, assess the effectiveness of cleanup and pollution control measures, and determine if discharges from all sources are protective of beneficial uses including aquatic life.

The objectives of this monitoring are to 1) establish a baseline for assessing continuous process improvement, 2) provide data for validation of proposed mixing zones and model verification, 3) provide data to inform the development of discharge limits and verify and validate discharge models, 4) assess total loading of all contaminants into the receiving water, 5) obtain data and information on toxicity for NPDES permit requirements for the Shipyard, and 6) develop procedures needed to meet ambient monitoring requirements for water, sediment, and biota in support of adaptive management actions.

This document presents the 2010 chemical residue data and stable isotopes of carbon $\left(\delta^{13} \mathrm{C}\right)$ and nitrogen $\left(\delta^{15} \mathrm{~N}\right)$ for the regional mussel watch stations located in Sinclair Inlet, Dyes Inlet, Port Orchard Passage, Rich Passage, Agate Passage, Liberty Bay, and Keyport Lagoon. Indigenous bivalves were collected from a small boat and/or from along the shoreline, measured, composited, and analyzed for a suite of trace metals and organic contaminants. The trace metals included silver $(\mathrm{Ag})$, arsenic (As), cadmium $(\mathrm{Cd})$, chromium $(\mathrm{Cr})$, copper $(\mathrm{Cu})$, mercury $(\mathrm{Hg})$, nickel $(\mathrm{Ni})$, lead $(\mathrm{Pb})$, and zinc $(\mathrm{Zn})$. The organic contaminants included the list of NOAA Status and Trends 20 polychlorinated biphenyls (PCB) congeners and suite of parent and methylated polycyclic aromatic hydrocarbons (PAHs). These chemical residue data provide the first year of the biota ambient monitoring.

For additional project information on biological monitoring see the following documents:

- 2003 Sinclair and Dyes Inlet TMDL Study: Biological Sampling and Analysis for Metals and PCBs (Brandenberger et al. 2003) 
- Biological Sampling and Analysis in Sinclair and Dyes Inlets, Washington: Chemical Analyses for 2005 Puget Sound Biota Study (Brandenberger et al. 2006a,b)

- Biological Sampling and Analysis in Sinclair and Dyes Inlets, Washington: Chemical Analyses for 2007 Puget Sound Biota Study (Brandenberger et al. 2008)

- Contaminate Residues in Demersal Fish, Invetebrates, and Deployed Mussels in Selected Areas of The Puget Sound, WA (Johnston et al. 2007).

- $\quad$ Sampling and Analysis Plan for Ambient Monitoring and Toxicity Testing for Sinclair and Dyes Inlets, Puget Sound, Washington (Johnston et al. 2009)

\section{Sample Collection and Analysis}

Twenty-four regional indigenous bivalve monitoring stations were established and sampled following procedures recommended by the National Oceanic and Atmospheric Administration (NOAA) Mussel Watch Program (NOAA 2009; SCMRC 2009) and documented in the ENVVEST ambient monitoring sampling and analysis plan (Johnston et al. 2009).. The target species for collection were blue mussels, Mytilus spp. (Mytilus trossulus, M. californianus, or M. galloprovincialis). Table 1 provides the station identification, site code for the database, and the coordinates for the location. The following information was recorded on the chain of custody sheet: station id, replicate number, date, time, and GPS coordinates of sampling location. A site description was also provided including, at a minimum, substrate type, habitat characteristics, presence/absence of creosote pilings, visible sheen of oil, and any other important factors associated with the collection. At each sampling location about 30-50 live specimens ( $>$ 1.5 inches) were collected at three replicate locations within a $150 \mathrm{ft}$ radius of the station location. The mussels will collected by cutting their byssus threads, brushing off as much sediment, barnacles, or other debris, and placing the mussels into labeled Ziploc bags along with water proof labels (station \#, bag \#, and collectors). Each station composite included from 123-577 specimens. The specimens were kept on ice until they were transferred to the Pacific Northwest National Marine Science Laboratory (MSL) for processing.

Mussels from 24 sampling locations were collected by the U.S. Navy and hand delivered to MSL. The live mussels were stored at $-20^{\circ} \mathrm{C}$ until they were measured and shucked. The length of each mussel added to the composite sample was recorded along with the total number of specimens in each composite (see Table 2). The mussels were rinsed with deionized water, shucked using a ceramic knife, and composites were homogenized to an even color and consistency using a titanium blender. The soft tissues from each of the three sampling areas representing a station were composited into each of three precleaned glass 8oz. jars. All jars were composited and ground in a pre-cleaned titanium blender to ensure homogenization. Aliquots were then placed into 1) 4 oz. pre-cleaned polypropylene jar for metals and isotopes, 2) 8oz. pre-cleaned glass jar for organics, and 3) $8 \mathrm{oz}$. pre-cleaned glass jar for archival. All aliquots were stored at $-20^{\circ} \mathrm{C}$. The aliquot for metals and stable isotopes was then lypholized and ground using a ball mill shaker system. An approximately $2 \mathrm{~g}$ aliquot of the dried and ground tissue was removed and 
sent to Dr. Jay Brandes, Skidaway Institute for Oceanography for $\delta^{13} \mathrm{C}$ and $\delta^{15} \mathrm{~N}$. Detailed descriptions of the analytical methods and quality control procedures for each parameter are provided in the quality control narrative accompanying each table. In summary, the dried, homogenized tissue was digested using an aqua regia mixture and analyzed for $\mathrm{Ag}$, $\mathrm{As}, \mathrm{Cd}$ and $\mathrm{Pb}$ using inductively coupled plasma mass spectrometry (ICP-MS); $\mathrm{Cr}, \mathrm{Cu}$, $\mathrm{Ni}$, and $\mathrm{Zn}$ using inductively coupled optical emissions spectroscopy (ICP-OES); and Hg by cold vapor atomic absorption (CVAA).

The $\delta^{13} \mathrm{C}$ and $\delta^{15} \mathrm{~N}$ analyses were performed on freeze dried, ground samples using a ThermoFinnigan Delta V plus stable isotope mass spectrometer coupled to a Thermo Flash elemental analyzer. Internal laboratory standards composed of marine chitin (Fisher Scientific) and calibrated to NIST standards were employed to correct sample data to international reference scales. Values are given vs. vPDB (C) and air (N) standard scales.

Tissue samples were extracted for PAHs and PCB congeners following MSL standard operating procedures based on the NOAA National Status and Trends (NS\&T) methods. Extracts intended for PAH analyses were analyzed using gas chromatography mass spectrometry (GC/MS) and extracts for PCB analyses were analyzed using gas chromatography with electron capture detection (GC/ECD). Sample data were quantified by the method of internal standards using the surrogate compounds. The list of organic compounds analyzed is as follows:

\begin{tabular}{|l|l|l|}
\hline PCBs & Parent PAHs & Methylated (alkylated) \\
\hline Cl2(8) & Naphthalene & C1-Naphthalenes \\
\hline C13(18) & Biphenyl & C2-Naphthalenes \\
\hline C13(28) & Acenaphthylene & C3-Naphthalenes \\
\hline C14(44) & Acenaphthene & C4-Naphthalenes \\
\hline C14(52) & Fluorene & C1-Fluorenes \\
\hline C14(66) & Anthracene & C2-Fluorenes \\
\hline C14(77) & Phenanthrene & C3-Fluorenes \\
\hline Cl5(101) & Dibenzothiophene & C1-Phenanthrenes/Anthracenes \\
\hline C15(105) & Fluoranthene & C2-Phenanthrenes/Anthracenes \\
\hline C15(118) & Pyrene & C3-Phenanthrenes/Anthracenes \\
\hline C15(126) & Benzo(a)anthracene & C4-Phenanthrenes/Anthracenes \\
\hline Cl6(128) & Chrysene & C1-Dibenzothiophenes \\
\hline C16(138) & Benzo(b)fluoranthene & C2-Dibenzothiophenes \\
\hline Cl6(153) & Benzo(k)fluoranthene & C3-Dibenzothiophenes \\
\hline C17(170) & Benzo(e)pyrene & C4-Dibenzothiophenes \\
\hline Cl7(180) & Benzo(a)pyrene & C1-Fluoranthenes/Pyrenes \\
\hline C17(187) & Perylene & C2-Fluoranthenes/Pyrenes \\
\hline C18(195) & Indeno(1,2,3-cd)pyrene & C3-Fluoranthenes/Pyrenes \\
\hline Cl8(200) & Dibenz(a,h)anthracene & C1-Chrysenes \\
\hline C19(206) & Benzo(g,h,i)perylene & C2-Chrysenes \\
\hline Cl10(209) & & C3-Chrysenes \\
\hline & & C4-Chrysenes \\
\hline
\end{tabular}




\begin{tabular}{|c|c|c|c|c|c|c|}
\hline Site Name & Site Code: & Latitude: & Longitude: & Station Description: & Substrate: & Creosote \\
\hline Manchester Lab Pier & MLPIER & 47.5736500 & -122.5450900 & $\begin{array}{l}\text { Clam Bay at Manchester Lab pier on floating } \\
\text { dock }\end{array}$ & Creosite piling & Yes \\
\hline $\begin{array}{l}\text { Sinclair Inlet Waterman } \\
\text { Point }\end{array}$ & SIWP & 47.5844700 & -122.5704400 & On rocks seaward of nav light, small mussels & rocks & No \\
\hline Sinclair Inlet Ross Point & SIRP & 47.5399200 & -122.6619000 & $\begin{array}{l}\text { Just east of point, rocky, cobble, \& sand. } \\
\text { Mussels embedded in beach face and on rocks }\end{array}$ & $\begin{array}{l}\text { rocky cobble and } \\
\text { sand }\end{array}$ & No \\
\hline $\begin{array}{l}\text { PSNS NAVSTA Mid } \\
(\mathrm{CP})\end{array}$ & PS04 & 47.5544920 & -122.6472150 & $\begin{array}{l}\text { East side of Charlie Pier on line hanging in } \\
\text { water }\end{array}$ & nylon line & Yes \\
\hline $\begin{array}{l}\text { PSNS NAVSTA West } \\
(\mathrm{DP})\end{array}$ & PS03 & 47.5559300 & -122.6513720 & $\begin{array}{l}\text { On steel cable at SW end of small boat dock } \\
\text { under stairs; Both samples from same location. }\end{array}$ & steel cable & Yes \\
\hline PSNS CIA West (6) & PS06 & 47.5529760 & -122.6426140 & $\begin{array}{l}\text { SE end of mole pier of DD6 near Pier B } \\
\text { construction. }\end{array}$ & steel cable & Yes \\
\hline PSNS CIA MidE (5) & PS08 & 47.5580000 & -122.6385000 & On barge near DD5 off mooring on steel cable & steel cable & Yes \\
\hline PSNS CIA MidW (4) & PS09 & 47.5601000 & -122.6363000 & $\begin{array}{l}\text { Off cement quay wall on steel cable haning in } \\
\text { water } \sim 15 \mathrm{ft} \text {. }\end{array}$ & steel cable & No \\
\hline PSNS CIA East (3) & PS11 & 47.5605000 & -122.6299000 & $\begin{array}{l}\text { Sampled from log and cable attached to quay } \\
\text { wall }\end{array}$ & log, steel cable & No \\
\hline $\begin{array}{l}\text { Sinclair Inlet Sinclair } \\
\text { Marina }\end{array}$ & SISIM & 47.5408000 & -122.6420500 & $\begin{array}{l}\text { Public pier east of marina on floating dock } \\
\text { from tires }\end{array}$ & rubber tire & No \\
\hline $\begin{array}{l}\text { Sinclair Inlet Port Orchard } \\
\text { Marina }\end{array}$ & SIPOM & 47.5431000 & -122.6355000 & $\begin{array}{l}\text { On foot ferry dock near passage to loading } \\
\text { platform }\end{array}$ & dock & No \\
\hline $\begin{array}{l}\text { Port Orchard Passage } \\
\text { Illahee State Park }\end{array}$ & POPISP & 47.5996000 & -122.5942700 & $\begin{array}{l}\text { On pier off floating dock and beach S. of pier } \\
\text { on woody debris (large log). }\end{array}$ & woody debris & No \\
\hline $\begin{array}{l}\text { Port Orchard Passage } \\
\text { Illahee Port Dock }\end{array}$ & POPIPD & 47.6126550 & -122.5953650 & $\begin{array}{l}\text { Illahee Port floating dock and under pier on } \\
\text { wooden crossbar }\end{array}$ & wood pier & No \\
\hline
\end{tabular}




\begin{tabular}{|c|c|c|c|c|c|c|}
\hline Site Name & Site Code: & Latitude: & Longitude: & Station Description: & Substrate: & Creosote \\
\hline $\begin{array}{l}\text { Port Washington Narrows } \\
\text { Lions Park }\end{array}$ & PWNLP & 47.5841610 & -122.6438430 & $\begin{array}{l}\text { On Northern edge of large extensive mussel } \\
\text { bed }\end{array}$ & $\begin{array}{l}\text { sand and cobbles } \\
\text { on intertidal mud } \\
\text { flat }\end{array}$ & No \\
\hline $\begin{array}{l}\text { Dyes Inlet Old Town } \\
\text { Silverdale }\end{array}$ & DYOTS & 47.6432000 & -122.6950000 & $\begin{array}{l}\text { Old Town Silverdale pier on pilings under pier } \\
\text { and floating dock }\end{array}$ & concrete piling & No \\
\hline $\begin{array}{l}\text { PSNS Inactive Fleet } \\
\text { Callow Ave OF }\end{array}$ & PS01 & 47.5536960 & -122.6574460 & $\begin{array}{l}\text { West end of Shipyard between carrier and } \\
\text { Charleston Beech near Callow Ave OF }\end{array}$ & electrical cable & No \\
\hline $\begin{array}{l}\text { Sinclair Inlet head at } \\
\text { Gorst }\end{array}$ & SIGST & 47.5330000 & -122.6800600 & $\begin{array}{l}\text { Head of Sinclair Inlet on western most } \\
\text { permanet mooring }\end{array}$ & steel mooring & No \\
\hline $\begin{array}{l}\text { Dyes Inlet Ostrich Bay } \\
\text { Ammo Pier }\end{array}$ & DYOBAP & 47.5864000 & -122.6868900 & $\begin{array}{l}\text { Dyes Inlet Ostrich Bay on end of abandonded } \\
\text { ammo pier at Jackson Park }\end{array}$ & concrete piling & No \\
\hline $\begin{array}{l}\text { Port Orchard Passage } \\
\text { Brownsville }\end{array}$ & POPBWN & 47.6524610 & -122.6125100 & Brownsville Marina in Port Orchard Passage & cement dock & No \\
\hline Keyport Lagoon & KPTLAG & 47.6967751 & -122.6190390 & $\begin{array}{l}\text { Keyport Lagoon inside lagoon near weir and } \\
\text { outside lagoon in shellfish bed and on concrete } \\
\text { radio tower base }\end{array}$ & $\begin{array}{l}\text { muddy beach; } \\
\text { shellfish bed, } \\
\text { cement tower }\end{array}$ & No \\
\hline Keyport NUWC Pier & KPTPIER & 47.7049500 & -122.6186800 & $\begin{array}{l}\text { NUWC Keyport pier on floating dock and steel } \\
\text { pilings }\end{array}$ & steel pilings & No \\
\hline $\begin{array}{l}\text { Liberty Bay Poulsbo } \\
\text { Marine Science Center }\end{array}$ & LBPMSC & 47.7323010 & -122.6487550 & Poulsbo Marina at end of guest dock & cement dock & No \\
\hline Agate Pass Kiana Lodge & APKIANA & 47.7017600 & -122.5812100 & $\begin{array}{l}\text { Suquamish Kiana Lodge at the end of floating } \\
\text { dock }\end{array}$ & dock & Yes \\
\hline $\begin{array}{l}\text { Agate Pass BI Hidden } \\
\text { Cove Beach }\end{array}$ & APHCB & 47.6908440 & -122.5662080 & $\begin{array}{l}\text { Bainbridge Island at the } \mathrm{W} \text { end of Hidden Cove } \\
\text { Rd. }\end{array}$ & rocks and cobbles & No \\
\hline
\end{tabular}


Table 2. ENVVEST AMB02 mussel watch collection information, March 2010.

\begin{tabular}{|c|c|c|c|c|}
\hline Sponsor ID & Station ID & $\begin{array}{c}\text { No. Mussels } \\
\text { in Comp }\end{array}$ & $\begin{array}{c}\text { Average } \\
\text { length }(\mathbf{m m})\end{array}$ & Stdev \\
\hline 20100112MUS01-C & PS04 & 194 & 45 & 7.9 \\
\hline 20100112MUS02-C & PS03 & 194 & 46 & 6.7 \\
\hline 20100112MUS03-C & PS06 & 329 & 47 & 7.3 \\
\hline 20100112MUS04-C & PS08 & 530 & 37 & 7.0 \\
\hline 20100112MUS05-C & PS09 & 123 & 49 & 9.3 \\
\hline 20100112MUS06-C & PS11 & 333 & 41 & 7.6 \\
\hline 20100112MUS07-C & SISIM & 179 & 48 & 12 \\
\hline 20100112MUS09-C & POPISP & 577 & 33 & 7.5 \\
\hline 20100112MUS08-C & SIPOM & 219 & 41 & 7.3 \\
\hline 20100112MUS10-C & POPIPD & 253 & 46 & 6.3 \\
\hline 20100112MUS11-C & PWNLP & 440 & 40 & 5.5 \\
\hline 20100112MUS12-C & DYOTS & 291 & 41 & 11 \\
\hline MW10EN01-C & MLPIER & 357 & 42 & 8.1 \\
\hline MW10EN03-C & SIWP & 300 & 26 & 5.0 \\
\hline MW10EN06-C & SIRP & 177 & 32 & 7.5 \\
\hline MFEB4-001C & POPBWN & 338 & 40 & 6.5 \\
\hline MFEB4-007C & KPTPIER & 273 & 38 & 9.2 \\
\hline MFEB4-013C & KPTLAG & 204 & 43 & 13 \\
\hline MFEB4-019C & APHCB & 342 & 26 & 3.7 \\
\hline MFEB4-023C & APKIANA & 248 & 44 & 7.4 \\
\hline MFEB4-029C & LBPMSC & 319 & 33 & 10 \\
\hline AMB02-301C & DYOBAP & 304 & 39 & 9.6 \\
\hline AMB02-295C & SIGST & 281 & 41 & 4.5 \\
\hline AMB02-102C & PS01 & 272 & 42 & 6.5 \\
\hline
\end{tabular}


Field Data Summary

2010 Regional Mussel Watch 
2010 Regional Mussel Watch - AMB02

PM: Jill Brandenberger 360/681-4564

ENVVEST 2010

Metals in Tissue

UNITS: $\mu \mathrm{g} / \mathrm{g}$ WET wt.

\begin{tabular}{|c|c|c|c|c|c|c|c|c|c|c|c|c|c|c|}
\hline Sample ID - Metals & Station Code & Fraction & $\begin{array}{l}\text { Sample } \\
\text { Type }\end{array}$ & MSL Code & $\begin{array}{l}\text { Collection } \\
\text { Date }\end{array}$ & $\begin{array}{c}\text { \# Mussels } \\
\text { in Comp }\end{array}$ & $\begin{array}{c}\text { Average } \\
\text { lenth } \\
(\mathrm{mm})\end{array}$ & $\begin{array}{l}\text { Percent } \\
\text { Moisture }\end{array}$ & Ag & As & Cd & $\mathbf{P b}$ & $\mathrm{Cr}$ & $\mathbf{C u}$ \\
\hline & & & & Instrument: & & & & & $I C P-M S$ & $I C P-M S$ & $I C P-M S$ & $I C P-M S$ & ICP-OES & ICP-OES \\
\hline \multirow{2}{*}{\multicolumn{8}{|c|}{$\begin{array}{l}\text { Laboratory Achieved Method Detection Limits (tissue) } \\
\text { Reporting Limit (MDL* 3.18) }\end{array}$}} & 85.9 & 0.00044 & 0.016 & 0.00041 & 0.00048 & 0.0049 & 0.013 \\
\hline & & & & & & & & & 0.001 & 0.05 & 0.0013 & 0.002 & 0.02 & 0.04 \\
\hline 20100112MUS01-C & PS04 & Composite & Mussels & $3106-215$ & $01 / 12 / 10$ & 194 & 45.3 & 86.6 & 0.00127 & 0.840 & 0.244 & 0.167 & 0.103 & 1.38 \\
\hline 20100112MUS02-C & PS03 & Composite & Mussels & $3106-216$ & $01 / 12 / 10$ & 194 & 45.6 & 88.2 & 0.00153 & 0.773 & 0.247 & 0.234 & 0.096 & 1.15 \\
\hline 20100112MUS03-C & PS06 & Composite & Mussels & $3106-217$ & $01 / 12 / 10$ & 329 & 47.3 & 87.8 & 0.00202 & 0.772 & 0.241 & 0.126 & 0.106 & 1.22 \\
\hline 20100112MUS04-C & PS08 & Composite & Mussels & $3106-218$ & $01 / 12 / 10$ & 530 & 37.1 & 88.3 & 0.00625 & 0.835 & 0.263 & 0.228 & 0.126 & 4.35 \\
\hline 20100112MUS05-C & PS09 & Composite & Mussels & $3106-219$ & $01 / 12 / 10$ & 123 & 48.7 & 88.7 & 0.00213 & 0.777 & 0.238 & 0.180 & 0.107 & 1.50 \\
\hline 20100112MUS06-C & PS11 & Composite & Mussels & $3106-220$ & $01 / 12 / 10$ & 333 & 41.0 & 87.9 & 0.00315 & 0.934 & 0.260 & 0.340 & 0.236 & 2.15 \\
\hline 20100112MUS07-C & SISIM & Composite & Mussels & $3106-221$ & $01 / 12 / 10$ & 179 & 48.0 & 88.6 & 0.00157 & 0.748 & 0.278 & 0.164 & 0.197 & 1.18 \\
\hline 20100112MUS09-C & POPISP & Composite & Mussels & $3106-222$ & $01 / 12 / 10$ & 577 & 33.0 & 82.3 & 0.00619 & 1.28 & 0.379 & 0.160 & 0.535 & 1.30 \\
\hline 20100112MUS08-C & SIPOM & Composite & Mussels & $3106-223$ & $01 / 12 / 10$ & 219 & 41.0 & 86.9 & 0.00142 & 0.857 & 0.284 & 0.135 & 0.111 & 1.81 \\
\hline 20100112MUS10-C & POPIPD & Composite & Mussels & $3106-224$ & $01 / 12 / 10$ & 253 & 46.0 & 83.8 & 0.00607 & 1.12 & 0.430 & 0.124 & 0.273 & 1.07 \\
\hline 20100112MUS11-C & PWNLP & Composite & Mussels & $3106-225$ & $01 / 12 / 10$ & 440 & 40.0 & 83.9 & 0.00606 & 1.07 & 0.289 & 0.259 & 0.387 & 1.24 \\
\hline 20100112MUS12-C & DYOTS & Composite & Mussels & $3106-226$ & $01 / 12 / 10$ & 291 & 41.0 & 83.4 & 0.00772 & 1.28 & 0.351 & 0.202 & 0.279 & 1.27 \\
\hline MW10EN01-C & MLPIER & Composite & Mussels & $3106-227$ & $01 / 08 / 10$ & 357 & 41.8 & 88.4 & 0.00311 & 1.01 & 0.489 & 0.074 & 0.107 & 0.828 \\
\hline MW10EN03-C & SIWP & Composite & Mussels & $3106-228$ & $01 / 08 / 10$ & 300 & 25.9 & 87.4 & 0.00424 & 1.07 & 0.295 & 0.269 & 0.206 & 1.08 \\
\hline MW10EN06-C & SIRP & Composite & Mussels & $3106-229$ & $01 / 08 / 10$ & 177 & 31.5 & 88.7 & 0.00373 & 0.892 & 0.299 & 0.221 & 0.359 & 1.24 \\
\hline MFEB4-001C & POPBWN & Composite & Mussels & $3106-230$ & $02 / 04 / 10$ & 338 & 40.0 & 85.5 & 0.00291 & 1.38 & 0.382 & 0.116 & 0.177 & 2.01 \\
\hline MFEB4-007C & KPTPIER & Composite & Mussels & $3106-231$ & $02 / 04 / 10$ & 273 & 38.0 & 83.6 & 0.00548 & 1.23 & 0.461 & 0.135 & 0.153 & 1.17 \\
\hline MFEB4-013C & KPTLAG & Composite & Mussels & $3106-232$ & $02 / 04 / 10$ & 204 & 43.0 & 88.0 & 0.00529 & 1.14 & 0.558 & 0.246 & 0.299 & 1.00 \\
\hline MFEB4-019C & APHCB & Composite & Mussels & $3106-233$ & $02 / 04 / 10$ & 342 & 26.0 & 84.9 & 0.00682 & 1.34 & 0.490 & 0.152 & 0.833 & 1.40 \\
\hline MFEB4-023C & APKIANA & Composite & Mussels & $3106-234$ & $02 / 04 / 10$ & 248 & 44.0 & 83.1 & 0.00700 & 1.94 & 0.568 & 0.109 & 0.182 & 1.45 \\
\hline MFEB4-029C & LBPMSC & Composite & Mussels & $3106-235$ & $02 / 04 / 10$ & 319 & 33.0 & 83.5 & 0.00318 & 1.57 & 0.437 & 0.198 & 0.205 & 3.92 \\
\hline AMB02-301C & DYOBAP & Composite & Mussels & $3106-236$ & $02 / 03 / 10$ & 304 & 39.0 & 83.5 & 0.00690 & 1.34 & 0.536 & 0.249 & 0.197 & 1.28 \\
\hline AMB02-295C & SIGST & Composite & Mussels & $3106-237$ & $02 / 03 / 10$ & 281 & 41.0 & 84.2 & 0.00304 & 0.833 & 0.232 & 0.136 & 0.127 & 1.80 \\
\hline AMB02-102C & PS01 & Composite & Mussels & $3106-238$ & $02 / 02 / 10$ & 272 & 42.0 & 84.8 & 0.00359 & 1.02 & 0.409 & 0.192 & 0.166 & 1.76 \\
\hline
\end{tabular}


BATTELLE MARINE SCIENCE LABORATORIES

1529 West Sequim Bay Road

Sequim, Washington 98382-9099

2010 Regional Mussel Watch - AMB02

PM: Jill Brandenberger 360/681-456

ENVVEST 2010

\begin{tabular}{|c|c|c|c|c|c|c|c|}
\hline Sample ID - Metals & \multirow[t]{2}{*}{ Station Code } & Fraction & $\begin{array}{l}\text { Sample } \\
\text { Type }\end{array}$ & MSL Code & $\mathbf{N i}$ & $\mathbf{Z n}$ & $\mathbf{H g}$ \\
\hline & & & & Instrument: & ICP-OES & ICP-OES & $C V A A$ \\
\hline \multirow{2}{*}{\multicolumn{5}{|c|}{ Laboratory Achieved Method Detection Limits (tissue) }} & 0.0058 & 0.0025 & 0.00062 \\
\hline & & & & & 0.02 & 0.008 & 0.002 \\
\hline 20100112MUS01-C & PS04 & Composite & Mussels & $3106-215$ & 0.0902 & 28.1 & 0.0152 \\
\hline 20100112MUS02-C & PS03 & Composite & Mussels & $3106-216$ & 0.0732 & 24.4 & 0.0465 \\
\hline 20100112MUS03-C & PS06 & Composite & Mussels & $3106-217$ & 0.0980 & 24.1 & 0.0146 \\
\hline 20100112MUS04-C & PS08 & Composite & Mussels & $3106-218$ & 0.128 & 31.5 & 0.0185 \\
\hline 20100112MUS05-C & PS09 & Composite & Mussels & $3106-219$ & 0.0964 & 23.7 & 0.0157 \\
\hline 20100112MUS06-C & PS11 & Composite & Mussels & $3106-220$ & 0.310 & 36.2 & 0.0164 \\
\hline 20100112MUS07-C & SISIM & Composite & Mussels & $3106-221$ & 0.170 & 23.0 & 0.0176 \\
\hline 20100112MUS09-C & POPISP & Composite & Mussels & $3106-222$ & 0.529 & 22.5 & 0.0158 \\
\hline 20100112MUS08-C & SIPOM & Composite & Mussels & $3106-223$ & 0.080 & 32.0 & 0.0180 \\
\hline 20100112MUS10-C & POPIPD & Composite & Mussels & $3106-224$ & 0.246 & 20.7 & 0.0136 \\
\hline 20100112MUS11-C & PWNLP & Composite & Mussels & $3106-225$ & 0.309 & 29.8 & 0.0232 \\
\hline 20100112MUS12-C & DYOTS & Composite & Mussels & $3106-226$ & 0.258 & 24.4 & 0.0288 \\
\hline MW10EN01-C & MLPIER & Composite & Mussels & $3106-227$ & 0.136 & 15.3 & 0.0109 \\
\hline MW10EN03-C & SIWP & Composite & Mussels & $3106-228$ & 0.206 & 24.7 & 0.0163 \\
\hline MW10EN06-C & SIRP & Composite & Mussels & $3106-229$ & 0.333 & 24.9 & 0.0221 \\
\hline MFEB4-001C & POPBWN & Composite & Mussels & $3106-230$ & 0.176 & 27.1 & 0.0184 \\
\hline MFEB4-007C & KPTPIER & Composite & Mussels & $3106-231$ & 0.150 & 25.5 & 0.0167 \\
\hline MFEB4-013C & KPTLAG & Composite & Mussels & $3106-232$ & 0.297 & 25.8 & 0.0176 \\
\hline MFEB4-019C & APHCB & Composite & Mussels & $3106-233$ & 0.542 & 28.7 & 0.0164 \\
\hline MFEB4-023C & APKIANA & Composite & Mussels & $3106-234$ & 0.198 & 27.2 & 0.0187 \\
\hline MFEB4-029C & LBPMSC & Composite & Mussels & $3106-235$ & 0.208 & 39.5 & 0.0231 \\
\hline AMB02-301C & DYOBAP & Composite & Mussels & $3106-236$ & 0.201 & 29.5 & 0.0253 \\
\hline AMB02-295C & SIGST & Composite & Mussels & $3106-237$ & 0.124 & 39.5 & 0.0141 \\
\hline AMB02-102C & PS01 & Composite & Mussels & $3106-238$ & 0.139 & 33.8 & 0.0200 \\
\hline
\end{tabular}


2010 Regional Mussel Watch - AMB02

PM: Jill Brandenberger 360/681-4564

ENVVEST 2010

NITS: $\mu$ g/o dry wt.

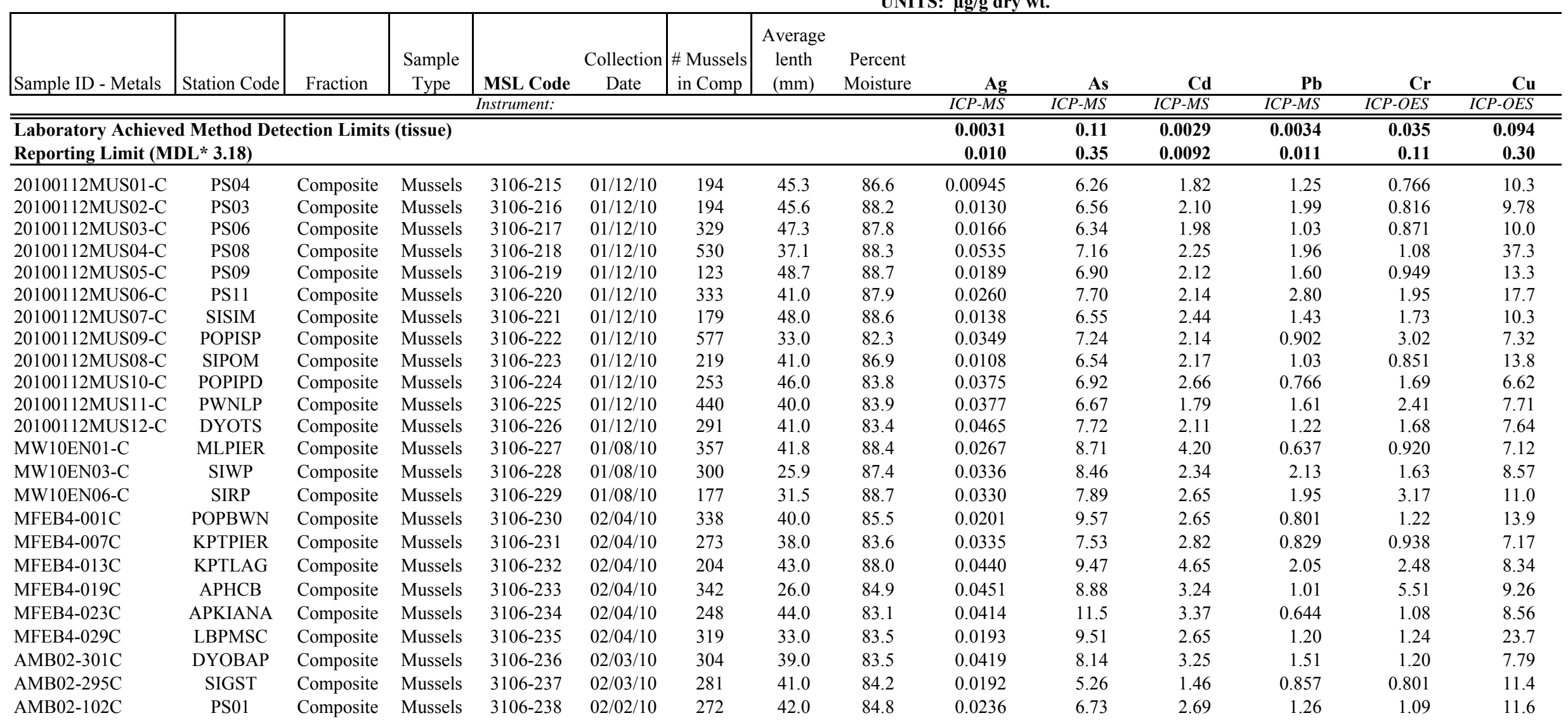


2010 Regional Mussel Watch - AMB02

PM: Jill Brandenberger 360/681-4564

ENVVEST 2010

Metals in Tissue

UNITS: $\mu \mathrm{g} / \mathrm{g}$ dry wt.

\begin{tabular}{|c|c|c|c|c|c|c|c|c|c|c|}
\hline Sample ID - Metals & Station Code & Fraction & $\begin{array}{l}\text { Sample } \\
\text { Type }\end{array}$ & MSL Code & $\mathbf{N i}$ & Zn & Hg & $\begin{array}{c}\text { CVAA } \\
\text { Batch ID }\end{array}$ & $\begin{array}{l}\text { ICP-OES } \\
\text { Batch ID }\end{array}$ & $\begin{array}{l}\text { ICP-MS } \\
\text { Batch ID }\end{array}$ \\
\hline & & & & Instrument: & ICP-OES & ICP-OES & CVAA & & & \\
\hline \multicolumn{5}{|c|}{$\begin{array}{l}\text { Laboratory Achieved Method Detection Limits (tissue) } \\
\end{array}$} & 0.041 & 0.018 & 0.0044 & & & \\
\hline \multicolumn{5}{|c|}{ Reporting Limit (MDL* 3.18) } & 0.13 & 0.057 & 0.014 & & & \\
\hline 20100112MUS01-C & PS04 & Composite & Mussels & $3106-215$ & 0.673 & 210 & 0.114 & 050610HGBL & I042310A & 042110-6100 \\
\hline 20100112MUS02-C & PS03 & Composite & Mussels & $3106-216$ & 0.622 & 207 & 0.395 & 050610HGBL & I042310A & $042110-6100$ \\
\hline 20100112MUS03-C & PS06 & Composite & Mussels & $3106-217$ & 0.804 & 198 & 0.120 & 050610HGBL & I042310A & $042110-6100$ \\
\hline 20100112MUS04-C & PS08 & Composite & Mussels & $3106-218$ & 1.09 & 270 & 0.159 & 050610HGBL & I042310A & $042110-6100$ \\
\hline 20100112MUS05-C & PS09 & Composite & Mussels & $3106-219$ & 0.857 & 210 & 0.140 & 050610HGBL & I042310A & 042110-6100 \\
\hline 20100112MUS06-C & PS11 & Composite & Mussels & $3106-220$ & 2.56 & 299 & 0.136 & 050610HGBL & I042310A & 042110-6100 \\
\hline 20100112MUS07-C & SISIM & Composite & Mussels & $3106-221$ & 1.49 & 202 & 0.154 & 050610HGBL & I042310A & 042110-6100 \\
\hline 20100112MUS09-C & POPISP & Composite & Mussels & $3106-222$ & 2.99 & 127 & 0.0893 & 050610HGBL & I042310A & 042110-6100 \\
\hline 20100112MUS08-C & SIPOM & Composite & Mussels & $3106-223$ & 0.607 & 244 & 0.138 & 050610HGBL & I042310A & 042110-6100 \\
\hline 20100112MUS10-C & POPIPD & Composite & Mussels & $3106-224$ & 1.52 & 128 & 0.0842 & 050610HGBL & I042310A & 042110-6100 \\
\hline 20100112MUS11-C & PWNLP & Composite & Mussels & $3106-225$ & 1.92 & 185 & 0.144 & 050610HGBL & I042310A & 042110-6100 \\
\hline 20100112MUS12-C & DYOTS & Composite & Mussels & $3106-226$ & 1.55 & 147 & 0.173 & 050610HGBL & I042310A & 042110-6100 \\
\hline MW10EN01-C & MLPIER & Composite & Mussels & $3106-227$ & 1.17 & 131 & 0.0935 & 050610HGBL & I042310A & 042110-6100 \\
\hline MW10EN03-C & SIWP & Composite & Mussels & $3106-228$ & 1.64 & 196 & 0.129 & 050610HGBL & I042310A & 042110-6100 \\
\hline MW10EN06-C & SIRP & Composite & Mussels & $3106-229$ & 2.95 & 221 & 0.196 & 050610HGBL & I042310A & 042110-6100 \\
\hline MFEB4-001C & POPBWN & Composite & Mussels & $3106-230$ & 1.22 & 188 & 0.127 & 050610HGBL & I042310A & 042110-6100 \\
\hline MFEB4-007C & KPTPIER & Composite & Mussels & $3106-231$ & 0.919 & 156 & 0.102 & 050610HGBL & I042310A & $042110-6100$ \\
\hline MFEB4-013C & KPTLAG & Composite & Mussels & $3106-232$ & 2.47 & 215 & 0.146 & 050610HGBL & I042310A & $042110-6100$ \\
\hline MFEB4-019C & APHCB & Composite & Mussels & $3106-233$ & 3.59 & 190 & 0.109 & 050610HGBL & I042310A & 042110-6100 \\
\hline MFEB4-023C & APKIANA & Composite & Mussels & $3106-234$ & 1.17 & 161 & 0.111 & 050610HGBL & I042310A & $042110-6100$ \\
\hline MFEB4-029C & LBPMSC & Composite & Mussels & $3106-235$ & 1.26 & 239 & 0.140 & 050610HGBL & I042310A & 042110-6100 \\
\hline AMB02-301C & DYOBAP & Composite & Mussels & $3106-236$ & 1.22 & 179 & 0.154 & 050610HGBL & I042310A & 042110-6100 \\
\hline AMB02-295C & SIGST & Composite & Mussels & $3106-237$ & 0.784 & 249 & 0.0891 & 050610HGBL & I042310A & $042110-6100$ \\
\hline AMB02-102C & PS01 & Composite & Mussels & $3106-238$ & 0.912 & 222 & 0.132 & 050610HGBL & I042310A & $042110-6100$ \\
\hline
\end{tabular}


BATTELLE MARINE SCIENCE LABORATORIES

1529 West Sequim Bay Road

Sequim, Washington 98382-9099

360/681-4564
2010 Regional Mussel Watch - AMB02
ENVVEST 2010

PCBs in Indigenous Mussels

UNITS: ng/g wet wt.

\begin{tabular}{|c|c|c|c|c|c|c|}
\hline Sample Name: & MDL & RL & 3106-200 & 3106-199 & $3106-198$ & 3106-197 \\
\hline$\overline{\text { Station: }}$ & & & POPIPD & 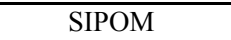 & POPISP & SISIM \\
\hline Sample Type: & & & Reg_Sample & Reg_Sample & Reg_Sample & Reg_Sample \\
\hline Matrix: & & & TISSUE & TISSUE & TISSUE & TISSUE \\
\hline Batch ID: & & & 070210PAH & 070210PAH & 070210PAH & 070210PAH \\
\hline Sample Weight (g): & & & 11.99 & 11.45 & 9.98 & 11.52 \\
\hline \%Moisture: & & & 83.83 & 86.90 & 82.29 & 88.58 \\
\hline Average \%Lipids (dry wt): & & & 8.40 & 7.78 & 8.85 & 6.99 \\
\hline Collection Date: & & & $1 / 12 / 2010$ & $1 / 12 / 2010$ & $1 / 12 / 2010$ & $1 / 12 / 2010$ \\
\hline Extraction Date: & & & $7 / 2 / 2010$ & $7 / 2 / 2010$ & $7 / 2 / 2010$ & $7 / 2 / 2010$ \\
\hline Analysis Date: & & & $7 / 8 / 2010$ & $7 / 8 / 2010$ & $7 / 8 / 2010$ & $7 / 8 / 2010$ \\
\hline Units (wet wt): & $\mathrm{ng} / \mathrm{g}$ & $\mathbf{n g} / \mathbf{g}$ & $\mathrm{ng} / \mathrm{g}$ & $\mathrm{ng} / \mathrm{g}$ & $\mathrm{ng} / \mathrm{g}$ & $\mathrm{ng} / \mathrm{g}$ \\
\hline $\mathrm{Cl} 2(8)$ & 0.16 & 0.2 & $0.19 \mathrm{~J}$ & 0.250 .24 & 0.26 & \\
\hline $\mathrm{Cl} 3(18)$ & 0.15 & 0.2 & $0.15 \mathrm{U}$ & 0.210 .16 & $\mathrm{~J}$ & 0.24 \\
\hline $\mathrm{Cl} 3(28)$ & 0.02 & 0.2 & $0.10 \mathrm{~J}$ & $0.20 \mathrm{~J}$ & $0.15 \mathrm{~J}$ & 0.22 \\
\hline $\mathrm{Cl} 4(44)$ & 0.02 & 0.2 & $0.14 \mathrm{~J}$ & $0.27 \quad 0.21$ & 0.34 & \\
\hline $\mathrm{Cl} 4(52)$ & 0.05 & 0.2 & $0.32 \quad 0.62$ & 0.48 & 0.77 & \\
\hline $\mathrm{Cl} 4(66)$ & 0.08 & 0.2 & $0.23 \quad 0.47$ & 0.30 & 0.70 & \\
\hline $\mathrm{Cl} 4(77)$ & 0.15 & 0.2 & $0.49 \quad 0.87$ & 0.65 & 1.08 & \\
\hline $\mathrm{Cl5}(101)$ & 0.17 & 0.2 & $1.00 \quad 1.83$ & 1.45 & 2.43 & \\
\hline $\mathrm{Cl}(105)$ & 0.03 & 0.2 & $0.24 \quad 0.56$ & 0.32 & 0.70 & \\
\hline $\mathrm{Cl5}(118)$ & 0.10 & 0.2 & $\begin{array}{ll}0.83 & 1.79\end{array}$ & 1.12 & 2.30 & \\
\hline $\mathrm{Cl}(126)$ & 0.07 & 0.2 & $0.67 \quad 0.86$ & 0.94 & 0.98 & \\
\hline $\mathrm{Cl}(128)$ & 0.04 & 0.2 & $0.29 \quad 0.41$ & 0.36 & 0.48 & \\
\hline Cl6(138) & 0.13 & 0.2 & $1.46 \quad 2.21$ & 1.98 & 2.79 & \\
\hline Cl6(153) & 0.18 & 0.2 & 1.792 .55 & 2.43 & 3.19 & \\
\hline $\mathrm{Cl} 7(170)$ & 0.02 & 0.2 & $0.09 \mathrm{~J}$ & $0.09 \mathrm{~J}$ & $0.14 \mathrm{~J}$ & $0.11 \mathrm{~J}$ \\
\hline $\mathrm{Cl}(180)$ & 0.03 & 0.2 & $0.25 \quad 0.35$ & 0.44 & 0.37 & \\
\hline $\mathrm{Cl}(187)$ & 0.08 & 0.2 & $0.72 \quad 0.92$ & 1.00 & 1.02 & \\
\hline $\mathrm{Cl}(195)$ & 0.01 & 0.2 & $0.01 \mathrm{U}$ & $0.01 \mathrm{U}$ & $0.01 \mathrm{U}$ & $0.01 \mathrm{U}$ \\
\hline $\mathrm{Cl}(200)$ & 0.08 & 0.2 & $0.26 \quad 0.35$ & 0.37 & 0.40 & \\
\hline $\mathrm{Cl}(206)$ & 0.01 & 0.2 & $0.01 \mathrm{U}$ & $0.01 \mathrm{U}$ & $0.01 \mathrm{U}$ & $0.01 \mathrm{U}$ \\
\hline Cl10(209) & 0.01 & 0.2 & $0.01 \mathrm{U}$ & $0.01 \mathrm{U}$ & $0.01 \mathrm{U}$ & $0.01 \mathrm{U}$ \\
\hline Sum PCB congeners ${ }^{1}$ & & & 18.46 & 29.63 & 25.49 & 36.80 \\
\hline \multicolumn{7}{|c|}{ SURROGATE RECOVERIES } \\
\hline $\mathrm{Cl} 3(30)$ & & & $89 \%$ & $87 \%$ & $76 \%$ & $75 \%$ \\
\hline $\mathrm{Cl} 4(65)$ & & & $96 \%$ & $99 \%$ & $87 \%$ & $84 \%$ \\
\hline $\mathrm{Cl}(198)$ & & & $92 \%$ & $75 \%$ & $70 \%$ & $74 \%$ \\
\hline
\end{tabular}

1 Sum of PCBs follows O'Conner (2002) and includes the MDL for non-detects 
BATTELLE MARINE SCIENCE LABORATORIES

1529 West Sequim Bay Road

Sequim, Washington 98382-9099

360/681-4564
2010 Regional Mussel Watch - AMB02

ENVVEST 2010

PCBs in Indigenous Mussels

UNITS: ng/g wet wt.

\begin{tabular}{|c|c|c|c|c|c|c|}
\hline Sample Name: & MDL & RL & 3106-191 & 3106-192 & $3106-193$ & 3106-194 \\
\hline Station: & & & 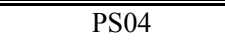 & $\overline{\mathrm{PS} 03}$ & PS06 & PS08 \\
\hline Sample Type: & & & Reg_Sample & Reg_Sample & Reg_Sample & Reg_Sample \\
\hline Matrix: & & & TISSUE & TISSUE & TISSUE & TISSUE \\
\hline Batch ID: & & & 070610PAH & 070610PAH & 070610PAH & 070610PAH \\
\hline Sample Weight (g): & & & 11.59 & 11.49 & 11.06 & 11.29 \\
\hline \%Moisture: & & & 86.49 & 88.23 & 87.82 & 88.33 \\
\hline Average \%Lipids (dry wt): & & & 6.87 & 6.62 & 6.65 & 7.47 \\
\hline Collection Date: & & & $1 / 12 / 2010$ & $1 / 12 / 2010$ & $1 / 12 / 2010$ & $1 / 12 / 2010$ \\
\hline Extraction Date: & & & $7 / 6 / 2010$ & $7 / 6 / 2010$ & $7 / 6 / 2010$ & $7 / 6 / 2010$ \\
\hline Analysis Date: & & & $8 / 3 / 2010$ & $8 / 3 / 2010$ & $8 / 3 / 2010$ & $8 / 3 / 2010$ \\
\hline Units (wet wt): & $\mathrm{ng} / \mathrm{g}$ & $\mathbf{n g} / \mathbf{g}$ & $\mathbf{n g} / \mathbf{g}$ & ng/g & ng/g & $\mathbf{n g} / \mathbf{g}$ \\
\hline $\mathrm{Cl} 2(8)$ & 0.16 & 0.2 & $0.32 \quad 0.31$ & 0.29 & 0.34 & \\
\hline $\mathrm{Cl} 3(18)$ & 0.15 & 0.2 & 0.310 .31 & 0.28 & 0.28 & \\
\hline $\mathrm{Cl} 3(28)$ & 0.02 & 0.2 & $0.21 \quad 0.26$ & 0.21 & 0.20 & \\
\hline $\mathrm{Cl}(44)$ & 0.02 & 0.2 & $0.31 \quad 0.37$ & 0.32 & 0.30 & \\
\hline $\mathrm{Cl} 4(52)$ & 0.05 & 0.2 & $0.77 \quad 0.84$ & 0.80 & 0.67 & \\
\hline Cl4(66) & 0.08 & 0.2 & 0.530 .60 & 0.63 & 0.48 & \\
\hline $\mathrm{Cl} 4(77)$ & 0.15 & 0.2 & $0.87 \quad 1.01$ & 1.05 & 1.06 & \\
\hline $\mathrm{Cl} 5(101)$ & 0.17 & 0.2 & $2.41 \quad 2.62$ & 2.60 & 2.37 & \\
\hline $\mathrm{Cl} 5(105)$ & 0.03 & 0.2 & $0.58 \quad 0.71$ & 0.73 & 0.80 & \\
\hline $\mathrm{Cl}(118)$ & 0.10 & 0.2 & 1.832 .26 & 2.24 & 2.26 & \\
\hline $\mathrm{Cl} 5(126)$ & 0.07 & 0.2 & $1.27 \quad 1.48$ & 1.46 & 1.41 & \\
\hline $\mathrm{Cl6}(128)$ & 0.04 & 0.2 & $0.47 \quad 0.57$ & 0.56 & 0.59 & \\
\hline Cl6(138) & 0.13 & 0.2 & $2.90 \quad 3.66$ & 3.43 & 3.52 & \\
\hline $\mathrm{Cl}(153)$ & 0.18 & 0.2 & $3.44 \quad 4.33$ & 3.97 & 3.74 & \\
\hline $\mathrm{Cl} 7(170)$ & 0.02 & 0.2 & $0.17 \mathrm{~J}$ & $0.19 \mathrm{~J}$ & $0.19 \mathrm{~J}$ & 0.22 \\
\hline $\mathrm{Cl} 7(180)$ & 0.03 & 0.2 & 0.690 .86 & 0.84 & 0.96 & \\
\hline $\mathrm{Cl}(187)$ & 0.08 & 0.2 & $1.31 \quad 1.64$ & 1.54 & 1.47 & \\
\hline $\mathrm{Cl}(195)$ & 0.01 & 0.2 & $0.01 \mathrm{U}$ & $0.01 \mathrm{U}$ & $0.01 \mathrm{U}$ & $0.01 \mathrm{U}$ \\
\hline $\mathrm{Cl} 8(200)$ & 0.08 & 0.2 & $0.54 \quad 0.65$ & 0.62 & 0.64 & \\
\hline $\mathrm{Cl}(206)$ & 0.01 & 0.2 & $0.01 \mathrm{U}$ & $0.01 \mathrm{U}$ & $0.01 \mathrm{U}$ & $0.01 \mathrm{U}$ \\
\hline Cl10(209) & 0.01 & 0.2 & $0.01 \mathrm{U}$ & $0.01 \mathrm{U}$ & $0.01 \mathrm{U}$ & $0.01 \mathrm{U}$ \\
\hline Sum PCB congeners ${ }^{1}$ & & & 37.90 & 45.36 & 43.57 & 42.64 \\
\hline \multicolumn{7}{|c|}{ SURROGATE RECOVERIES } \\
\hline $\mathrm{Cl} 3(30)$ & & & $82 \%$ & $91 \%$ & $90 \%$ & $84 \%$ \\
\hline $\mathrm{Cl}(4(65)$ & & & $90 \%$ & $100 \%$ & $99 \%$ & $93 \%$ \\
\hline $\mathrm{Cl}(198)$ & & & $73 \%$ & $75 \%$ & $74 \%$ & $69 \%$ \\
\hline
\end{tabular}


BATTELLE MARINE SCIENCE LABORATORIES

1529 West Sequim Bay Road

Sequim, Washington 98382-9099

360/681-4564
2010 Regional Mussel Watch - AMB02

ENVVEST 2010

PCBs in Indigenous Mussels

UNITS: ng/g wet wt.

\begin{tabular}{|c|c|c|c|c|c|c|}
\hline Sample Name: & MDL & RL & 3106-195 & 3106-196 & 3106-201 & 3106-202 \\
\hline Station: & & & PS09 & PS11 & PWNLP & DYOTS \\
\hline Sample Type: & & & Reg_Sample & Reg_Sample & Reg_Sample & Reg_Sample \\
\hline Matrix: & & & TISSUE & TISSUE & TISSUE & TISSUE \\
\hline Batch ID: & & & 070610PAH & 070610PAH & 071910PAH & 071910PAH \\
\hline Sample Weight (g): & & & 11.86 & 12.46 & 11.4 & 13.26 \\
\hline \%Moisture: & & & 88.74 & 87.88 & 83.92 & 83.37 \\
\hline Average \%Lipids (dry wt): & & & 6.24 & 6.96 & 9.40 & 8.61 \\
\hline Collection Date: & & & $1 / 12 / 2010$ & $1 / 12 / 2010$ & $1 / 12 / 2010$ & $1 / 12 / 2010$ \\
\hline Extraction Date: & & & $7 / 6 / 2010$ & $7 / 6 / 2010$ & $7 / 19 / 2010$ & $7 / 19 / 2010$ \\
\hline Analysis Date: & & & $8 / 3 / 2010$ & $8 / 3 / 2010$ & $8 / 4 / 2010$ & $8 / 4 / 2010$ \\
\hline Units (wet wt): & $\mathbf{n g} / \mathrm{g}$ & $\mathrm{ng} / \mathrm{g}$ & $\mathrm{ng} / \mathrm{g}$ & $\mathrm{ng} / \mathrm{g}$ & $\mathrm{ng} / \mathrm{g}$ & $\mathrm{ng} / \mathrm{g}$ \\
\hline $\mathrm{Cl} 2(8)$ & 0.16 & 0.2 & $0.46 \quad 0.23$ & 0.22 & & 0.30 \\
\hline $\mathrm{Cl} 3(18)$ & 0.15 & 0.2 & $0.45 \quad 0.22$ & 0.15 & $\mathrm{U}$ & 0.21 \\
\hline $\mathrm{Cl} 3(28)$ & 0.02 & 0.2 & $0.23 \quad 0.20$ & $\mathrm{~J}$ & $0.11 \mathrm{~J}$ & $0.16 \mathrm{~J}$ \\
\hline Cl4(44) & 0.02 & 0.2 & $0.49 \quad 0.26$ & 0.17 & $\mathrm{~J}$ & 0.22 \\
\hline $\mathrm{Cl} 4(52)$ & 0.05 & 0.2 & $1.07 \quad 0.65$ & 0.41 & & 0.55 \\
\hline Cl4(66) & 0.08 & 0.2 & $0.80 \quad 0.49$ & 0.28 & & 0.40 \\
\hline $\mathrm{Cl} 4(77)$ & 0.15 & 0.2 & $\begin{array}{ll}1.89 & 1.09\end{array}$ & 0.73 & & 0.82 \\
\hline $\mathrm{Cl} 5(101)$ & 0.17 & 0.2 & $5.11 \quad 2.54$ & 1.50 & & 1.68 \\
\hline $\mathrm{Cl} 5(105)$ & 0.03 & 0.2 & $1.33 \quad 0.75$ & 0.41 & & 0.45 \\
\hline $\mathrm{Cl} 5(118)$ & 0.10 & 0.2 & $4.10 \quad 2.39$ & 1.41 & & 1.54 \\
\hline $\mathrm{Cl} 5(126)$ & 0.07 & 0.2 & $3.04 \quad 1.52$ & 1.05 & & 0.99 \\
\hline $\mathrm{Cl} 6(128)$ & 0.04 & 0.2 & $\begin{array}{ll}0.97 & 0.59\end{array}$ & 0.38 & & 0.42 \\
\hline $\mathrm{Cl6}(138)$ & 0.13 & 0.2 & $8.13 \quad 3.78$ & 2.30 & & 2.33 \\
\hline $\mathrm{Cl}(153)$ & 0.18 & 0.2 & $10.67 \quad 4.26$ & 2.87 & & 2.85 \\
\hline $\mathrm{Cl}(170)$ & 0.02 & 0.2 & $0.44 \quad 0.18$ & $\mathrm{~J}$ & $0.13 \mathrm{~J}$ & $0.09 \mathrm{~J}$ \\
\hline $\mathrm{Cl}(180)$ & 0.03 & 0.2 & $2.14 \quad 0.87$ & 0.40 & & 0.28 \\
\hline $\mathrm{Cl} 7(187)$ & 0.08 & 0.2 & $3.13 \quad 1.59$ & 1.12 & & 1.05 \\
\hline $\mathrm{Cl}(195)$ & 0.01 & 0.2 & $0.01 \mathrm{U}$ & $0.01 \mathrm{U}$ & $0.01 \mathrm{U}$ & $0.01 \mathrm{U}$ \\
\hline $\mathrm{Cl}(200)$ & 0.08 & 0.2 & 1.310 .65 & 0.41 & & 0.38 \\
\hline $\mathrm{Cl}(206)$ & 0.01 & 0.2 & $0.01 \mathrm{U}$ & $0.01 \mathrm{U}$ & $0.01 \mathrm{U}$ & $0.01 \mathrm{U}$ \\
\hline Cl10(209) & 0.01 & 0.2 & $0.01 \mathrm{U}$ & $0.01 \mathrm{U}$ & $0.01 \mathrm{U}$ & $0.01 \mathrm{U}$ \\
\hline Sum PCB congeners ${ }^{1}$ & & & 91.54 & 44.56 & 28.09 & 29.48 \\
\hline \multicolumn{7}{|c|}{ SURROGATE RECOVERIES } \\
\hline $\mathrm{Cl} 3(30)$ & & & $84 \%$ & $83 \%$ & $85 \%$ & $84 \%$ \\
\hline $\mathrm{Cl} 4(65)$ & & & $92 \%$ & $92 \%$ & $98 \%$ & $94 \%$ \\
\hline $\mathrm{Cl}(198)$ & & & $66 \%$ & $64 \%$ & $67 \%$ & $68 \%$ \\
\hline
\end{tabular}

1 Sum of PCBs follows O'Conner (2002) and incl 
BATTELLE MARINE SCIENCE LABORATORIES

1529 West Sequim Bay Road

Sequim, Washington 98382-9099

360/681-4564

\section{Regional Mussel Watch - AMB02 \\ ENVVEST 2010}

PCBs in Indigenous Mussels

UNITS: ng/g wet wt.

\begin{tabular}{|c|c|c|c|c|c|c|}
\hline Sample Name: & MDL & $\overline{R L}$ & $3106-203$ & 3106-204 & 3106-205 & $3106-206$ \\
\hline Station: & & & MLPIER & 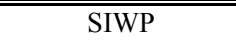 & 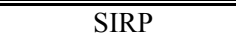 & 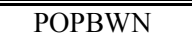 \\
\hline Sample Type: & & & Reg_Sample & Reg_Sample & Reg_Sample & Reg_Sample \\
\hline Matrix: & & & TISSUE & TISSUE & TISSUE & TISSUE \\
\hline Batch ID: & & & 071910PAH & 071910PAH & 071910PAH & 071910PAH \\
\hline Sample Weight (g): & & & 13.1 & 11.59 & 11.82 & 12.08 \\
\hline \%Moisture: & & & 88.37 & 87.37 & 88.69 & 85.55 \\
\hline Average \%Lipids (dry wt): & & & 6.40 & 5.43 & 5.42 & 8.07 \\
\hline Collection Date: & & & $1 / 8 / 2010$ & $1 / 8 / 2010$ & $1 / 8 / 2010$ & $2 / 4 / 2010$ \\
\hline Extraction Date: & & & $7 / 19 / 2010$ & $7 / 19 / 2010$ & $7 / 19 / 2010$ & $7 / 19 / 2010$ \\
\hline Analysis Date: & & & $8 / 4 / 2010$ & $8 / 4 / 2010$ & $8 / 4 / 2010$ & $8 / 4 / 2010$ \\
\hline Units (wet wt): & ng/g & $\mathrm{ng} / \mathrm{g}$ & $\mathrm{ng} / \mathrm{g}$ & ng/g & $\mathrm{ng} / \mathrm{g}$ & ng/g \\
\hline $\mathrm{Cl} 2(8)$ & 0.16 & 0.2 & $0.18 \mathrm{~J}$ & $0.17 \mathrm{~J}$ & $0.20 \quad 0.20$ & $\mathrm{~J}$ \\
\hline $\mathrm{Cl} 3(18)$ & 0.15 & 0.2 & $0.18 \mathrm{~J}$ & $0.15 \mathrm{U}$ & $0.16 \mathrm{~J}$ & $0.17 \mathrm{~J}$ \\
\hline $\mathrm{Cl} 3(28)$ & 0.02 & 0.2 & $0.07 \mathrm{~J}$ & $0.08 \mathrm{~J}$ & $0.12 \mathrm{~J}$ & $0.17 \mathrm{~J}$ \\
\hline $\mathrm{Cl} 4(44)$ & 0.02 & 0.2 & $0.13 \mathrm{~J}$ & $0.10 \mathrm{~J}$ & $0.15 \mathrm{~J}$ & $0.18 \mathrm{~J}$ \\
\hline $\mathrm{Cl} 4(52)$ & 0.05 & 0.2 & $0.31 \quad 0.25$ & 0.41 & 0.41 & \\
\hline $\mathrm{Cl} 4(66)$ & 0.08 & 0.2 & $0.17 \mathrm{~J}$ & $0.14 \mathrm{~J}$ & 0.290 .35 & \\
\hline $\mathrm{Cl} 4(77)$ & 0.15 & 0.2 & $0.47 \quad 0.39$ & 0.64 & 0.54 & \\
\hline $\mathrm{Cl} 5(101)$ & 0.17 & 0.2 & 1.180 .82 & 1.63 & 1.19 & \\
\hline $\mathrm{Cl} 5(105)$ & 0.03 & 0.2 & $0.19 \mathrm{~J}$ & $0.20 \mathrm{~J}$ & $0.47 \quad 0.31$ & \\
\hline $\mathrm{Cl5}(118)$ & 0.10 & 0.2 & $0.81 \quad 0.78$ & 1.46 & 1.07 & \\
\hline $\mathrm{Cl}(126)$ & 0.07 & 0.2 & 1.600 .68 & 1.04 & 0.71 & \\
\hline $\mathrm{Cl}(128)$ & 0.04 & 0.2 & $0.31 \quad 0.24$ & 0.38 & 0.29 & \\
\hline $\mathrm{Cl}(138)$ & 0.13 & 0.2 & $2.48 \quad 1.33$ & 2.23 & 1.63 & \\
\hline Cl6(153) & 0.18 & 0.2 & $3.46 \quad 1.70$ & 2.73 & 2.01 & \\
\hline $\mathrm{Cl}(170)$ & 0.02 & 0.2 & $0.17 \mathrm{~J}$ & $0.11 \mathrm{~J}$ & $0.13 \mathrm{~J}$ & $0.06 \mathrm{~J}$ \\
\hline $\mathrm{Cl}(180)$ & 0.03 & 0.2 & $\begin{array}{ll}0.88 & 0.26\end{array}$ & 0.42 & 0.23 & \\
\hline $\mathrm{Cl}(187)$ & 0.08 & 0.2 & 1.620 .71 & 1.04 & 0.74 & \\
\hline $\mathrm{Cl}(195)$ & 0.01 & 0.2 & $0.01 \mathrm{U}$ & $0.01 \mathrm{U}$ & $0.01 \mathrm{U}$ & $0.01 \mathrm{U}$ \\
\hline $\mathrm{Cl}(200)$ & 0.08 & 0.2 & $0.73 \quad 0.24$ & 0.42 & 0.26 & \\
\hline $\mathrm{Cl}$ (206) & 0.01 & 0.2 & $0.01 \mathrm{U}$ & $0.01 \mathrm{U}$ & $0.01 \mathrm{U}$ & $0.01 \mathrm{U}$ \\
\hline Cl10(209) & 0.01 & 0.2 & $0.01 \mathrm{U}$ & $0.01 \mathrm{U}$ & $0.01 \mathrm{U}$ & $0.01 \mathrm{U}$ \\
\hline Sum PCB congeners ${ }^{1}$ & & & 29.92 & 16.73 & 27.89 & 21.07 \\
\hline \multicolumn{7}{|c|}{ SURROGATE RECOVERIES } \\
\hline $\mathrm{Cl} 3(30)$ & & & $75 \%$ & $78 \%$ & $74 \%$ & $65 \%$ \\
\hline $\mathrm{Cl}(4)$ & & & $82 \%$ & $92 \%$ & $85 \%$ & $78 \%$ \\
\hline $\mathrm{Cl}(198)$ & & & $70 \%$ & $70 \%$ & $64 \%$ & $58 \%$ \\
\hline
\end{tabular}

1 Sum of PCBs follows O'Conner (2002) and inclı 
BATTELLE MARINE SCIENCE LABORATORIES

1529 West Sequim Bay Road

Sequim, Washington 98382-9099

360/681-4564
2010 Regional Mussel Watch - AMB02

ENVVEST 2010

PCBs in Indigenous Mussels

UNITS: $n g / g$ wet wt.

\begin{tabular}{|c|c|c|c|c|c|c|}
\hline Sample Name: & MDL & RL & 3107-207 & $3106-208$ & 3106-209 & $3106-210$ \\
\hline Station: & & & "KPTPIER & KPTLAG & APHCB & APKIANA \\
\hline Sample Type: & & & Reg_Sample & Reg_Sample & Reg_Sample & Reg_Sample \\
\hline Matrix: & & & TISSUE & TISSUE & TISSUE & TISSUE \\
\hline Batch ID: & & & 071910PAH & 071910PAH & 071910PAH & 072910PAH \\
\hline Sample Weight (g): & & & 11.62 & 12.8 & 11.6 & 11.63 \\
\hline \%Moisture: & & & 83.65 & 87.99 & 84.88 & 83.12 \\
\hline Average \%Lipids (dry wt): & & & 7.92 & 7.55 & 5.52 & 7.57 \\
\hline Collection Date: & & & $2 / 4 / 2010$ & $2 / 4 / 2010$ & $2 / 4 / 2010$ & $2 / 4 / 2010$ \\
\hline Extraction Date: & & & $7 / 19 / 2010$ & $7 / 19 / 2010$ & $7 / 19 / 2010$ & $7 / 29 / 2010$ \\
\hline Analysis Date: & & & $8 / 4 / 2010$ & $8 / 4 / 2010$ & $8 / 6 / 2010$ & $8 / 6 / 2010$ \\
\hline Units (wet wt): & $\mathbf{n g} / \mathrm{g}$ & $\mathbf{n g} / \mathbf{g}$ & $\mathrm{ng} / \mathrm{g}$ & $\mathrm{ng} / \mathrm{g}$ & $\mathrm{ng} / \mathrm{g}$ & $\mathbf{n g} / \mathbf{g}$ \\
\hline $\mathrm{Cl} 2(8)$ & 0.16 & 0.2 & 0.250 .21 & 0.17 & $\mathrm{~J}$ & $0.18 \mathrm{~J}$ \\
\hline $\mathrm{Cl} 3(18)$ & 0.15 & 0.2 & $0.18 \mathrm{~J}$ & $0.15 \mathrm{U}$ & $0.15 \mathrm{U}$ & $0.15 \mathrm{U}$ \\
\hline $\mathrm{Cl} 3(28)$ & 0.02 & 0.2 & $0.17 \mathrm{~J}$ & $0.10 \mathrm{~J}$ & $0.08 \mathrm{~J}$ & $0.10 \mathrm{~J}$ \\
\hline Cl4(44) & 0.02 & 0.2 & $0.18 \mathrm{~J}$ & $0.12 \mathrm{~J}$ & $0.12 \mathrm{~J}$ & $0.13 \mathrm{~J}$ \\
\hline $\mathrm{Cl} 4(52)$ & 0.05 & 0.2 & $0.48 \quad 0.29$ & 0.24 & 0.32 & \\
\hline Cl4(66) & 0.08 & 0.2 & $\begin{array}{lll}0.31 & 0.19\end{array}$ & $\mathrm{~J}$ & $0.12 \mathrm{~J}$ & $0.20 \mathrm{~J}$ \\
\hline Cl4(77) & 0.15 & 0.2 & $\begin{array}{lll}0.67 & 0.45\end{array}$ & 0.36 & 0.41 & \\
\hline $\mathrm{Cl5}(101)$ & 0.17 & 0.2 & $1.40 \quad 1.00$ & 0.78 & 1.03 & \\
\hline $\mathrm{Cl} 5(105)$ & 0.03 & 0.2 & $\begin{array}{lll}0.35 & 0.27\end{array}$ & 0.19 & $\mathrm{~J}$ & 0.25 \\
\hline Cl5(118) & 0.10 & 0.2 & 1.200 .95 & 0.65 & 0.81 & \\
\hline $\mathrm{Cl} 5(126)$ & 0.07 & 0.2 & $0.73 \quad 0.58$ & 0.56 & 0.62 & \\
\hline Cl6(128) & 0.04 & 0.2 & $0.34 \quad 0.26$ & 0.21 & 0.29 & \\
\hline $\mathrm{Cl6}(138)$ & 0.13 & 0.2 & $1.81 \quad 1.45$ & 1.23 & 1.54 & \\
\hline $\mathrm{Cl}(153)$ & 0.18 & 0.2 & $2.16 \quad 1.74$ & 1.53 & 1.93 & \\
\hline $\mathrm{Cl}(170)$ & 0.02 & 0.2 & $0.08 \mathrm{~J}$ & $0.06 \mathrm{~J}$ & $0.07 \mathrm{~J}$ & $0.08 \mathrm{~J}$ \\
\hline $\mathrm{Cl} 17(180)$ & 0.03 & 0.2 & $0.20 \quad 0.15$ & $\mathrm{~J}$ & $0.22 \quad 0.26$ & \\
\hline Cl7(187) & 0.08 & 0.2 & $\begin{array}{lll}0.77 & 0.61\end{array}$ & 0.60 & 0.72 & \\
\hline $\mathrm{Cl}(195)$ & 0.01 & 0.2 & $0.01 \mathrm{U}$ & $0.01 \mathrm{U}$ & $0.01 \mathrm{U}$ & $0.01 \mathrm{U}$ \\
\hline $\mathrm{Cl} 8(200)$ & 0.08 & 0.2 & $0.27 \quad 0.20$ & $\mathrm{~J}$ & $0.19 \mathrm{~J}$ & 0.22 \\
\hline $\mathrm{Cl9}(206)$ & 0.01 & 0.2 & $0.01 \mathrm{U}$ & $0.01 \mathrm{U}$ & $0.01 \mathrm{U}$ & $0.01 \mathrm{U}$ \\
\hline Cl10(209) & 0.01 & 0.2 & $0.01 \mathrm{U}$ & $0.01 \mathrm{U}$ & $0.01 \mathrm{U}$ & $0.01 \mathrm{U}$ \\
\hline Sum PCB congeners ${ }^{1}$ & & & 23.15 & 17.60 & 14.99 & 18.52 \\
\hline \multicolumn{7}{|c|}{ SURROGATE RECOVERIES } \\
\hline $\mathrm{Cl} 3(30)$ & & & $81 \%$ & $81 \%$ & $63 \%$ & $85 \%$ \\
\hline $\mathrm{Cl} 4(65)$ & & & $91 \%$ & $88 \%$ & $77 \%$ & $94 \%$ \\
\hline $\mathrm{Cl} 18(198)$ & & & $68 \%$ & $64 \%$ & $64 \%$ & $70 \%$ \\
\hline
\end{tabular}


BATTELLE MARINE SCIENCE LABORATORIES

1529 West Sequim Bay Road

Sequim, Washington 98382-9099

360/681-4564
2010 Regional Mussel Watch - AMB02

ENVVEST 2010

PCBs in Indigenous Mussels

UNITS: ng/g wet wt.

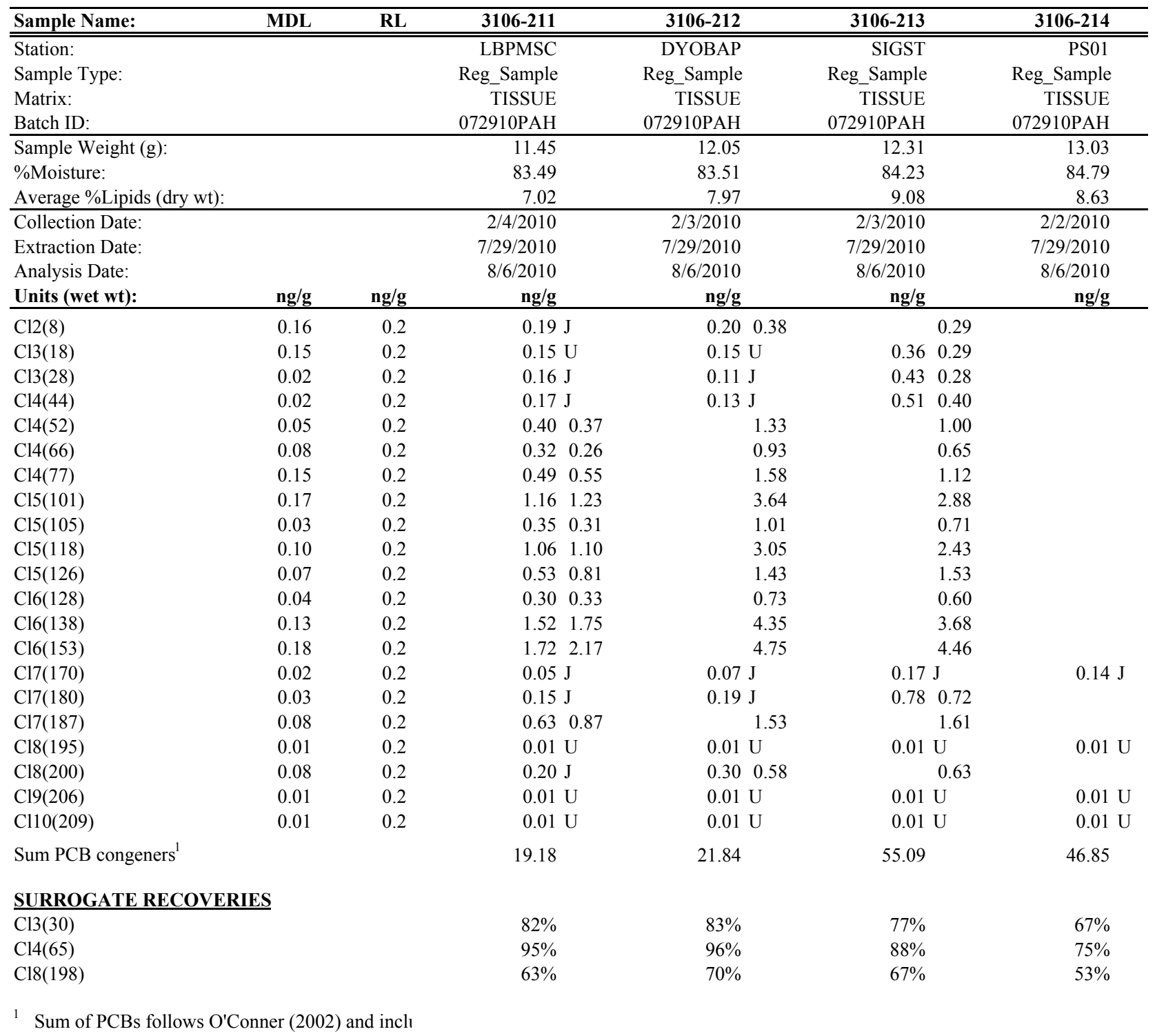


BATTELLE MARINE SCIENCE LABORATORIES

1529 West Sequim Bay Road

Sequim, Washington 98382-9099

360/681-4564
2010 Regional Mussel Watch - AMB02

ENVVEST 2010

PAHs in Indigenous Mussels UNITS: ng/g wet wt.

\begin{tabular}{|c|c|c|c|c|c|c|}
\hline Sample Name: & & & $3106-200$ & 3106-199 & 3106-198 & 3106-197 \\
\hline Station: & & & POPIPD & SIPOM & POPISP & SISIM \\
\hline Sample Type: & & & Reg_Sample & Reg_Sample & Reg_Sample & Reg_Sample \\
\hline Matrix: & & & TISSUE & TISSUE & TISSUE & TISSUE \\
\hline Batch ID: & & & 070210PAH & 070210PAH & 070210PAH & 070210PAH \\
\hline Sample Weight (g): & & & 11.99 & 11.45 & 9.98 & 11.52 \\
\hline \%Moisture: & & & 83.83 & 86.90 & 82.29 & 88.58 \\
\hline Average \%Lipids (dry wt): & & & 8.40 & 7.78 & 8.85 & 6.99 \\
\hline Collection Date: & & & $1 / 12 / 2010$ & $1 / 12 / 2010$ & $1 / 12 / 2010$ & $1 / 12 / 2010$ \\
\hline Extraction Date: & & & $7 / 2 / 2010$ & $7 / 2 / 2010$ & $7 / 2 / 2010$ & $7 / 2 / 2010$ \\
\hline Analysis Date: & & & $8 / 12 / 2010$ & $8 / 12 / 2010$ & $8 / 12 / 2010$ & $8 / 12 / 2010$ \\
\hline Units (wet wt): & MDL (ng/g) & RL & ng/g & ng/g & ng/g & $\mathbf{n g} / \mathrm{g}$ \\
\hline Naphthalene & 1.82 & 1.82 & $5.73 \mathrm{~B}$ & $5.00 \mathrm{~B}$ & $31.4 \mathrm{~B}$ & $4.10 \mathrm{~B}$ \\
\hline C1-Naphthalenes & 1.82 & 1.82 & $3.71 \mathrm{~B}$ & $5.44 \mathrm{~B}$ & $34.5 \mathrm{~B}$ & $4.22 \mathrm{~B}$ \\
\hline C2-Naphthalenes & 3.61 & 1.82 & 9.1911 .3 & & 35.8 & 8.81 \\
\hline C3-Naphthalenes & 3.61 & 1.82 & 7.6919 .4 & & 23.2 & 9.86 \\
\hline C4-Naphthalenes & 3.61 & 1.82 & $4.72 \quad 21.6$ & & 9.00 & 7.44 \\
\hline Biphenyl & 2.86 & 1.82 & $2.86 \mathrm{U}$ & $2.86 \mathrm{U}$ & 9.892 .86 & $\mathrm{U}$ \\
\hline Acenaphthylene & 1.68 & 1.82 & $1.68 \mathrm{U}$ & $1.68 \mathrm{U}$ & 4.172 .45 & \\
\hline Acenaphthene & 2.29 & 1.82 & $3.48 \quad 2.29$ & $\mathrm{U}$ & 62.9 & $2.29 \mathrm{U}$ \\
\hline Fluorene & 2.75 & 1.82 & $3.98 \quad 2.75$ & $\mathrm{U}$ & 39.2 & $2.75 \mathrm{U}$ \\
\hline C1-Fluorenes & 2.17 & 1.82 & 16.416 .6 & & 26.6 & 11.7 \\
\hline C2-Fluorenes & 2.17 & 1.82 & $2.17 \mathrm{U}$ & $2.17 \mathrm{U}$ & $2.17 \mathrm{U}$ & $2.17 \mathrm{U}$ \\
\hline C3-Fluorenes & 2.17 & 1.82 & $2.17 \mathrm{U}$ & $2.17 \mathrm{U}$ & $2.17 \mathrm{U}$ & $2.17 \mathrm{U}$ \\
\hline Anthracene & 0.42 & 1.82 & 5.493 .29 & & 12.2 & 5.47 \\
\hline Phenanthrene & 1.10 & 1.82 & 17.418 .0 & & 55.7 & 21.0 \\
\hline C1-Phenanthrenes/Anthracenes & 1.18 & 1.82 & $63.8 \quad 50.5$ & & 83.9 & 41.4 \\
\hline C2-Phenanthrenes/Anthracenes & 0.10 & 1.82 & $13.7 \quad 62.4$ & & 20.8 & 26.1 \\
\hline C3-Phenanthrenes/Anthracenes & 0.10 & 1.82 & $16.0 \quad 39.9$ & & 43.4 & 20.2 \\
\hline C4-Phenanthrenes/Anthracenes & 0.10 & 1.82 & $0.10 \mathrm{U}$ & $33.9 \quad 0.10$ & $\mathrm{U}$ & $0.10 \mathrm{U}$ \\
\hline Dibenzothiophene & 0.40 & 1.82 & $0.68 \mathrm{~J}$ & $1.08 \mathrm{~J}$ & 4.180 .88 & $\mathrm{~J}$ \\
\hline C1-Dibenzothiophenes & 0.40 & 1.82 & $1.65 \mathrm{~J}$ & $3.30 \quad 2.83$ & & $1.56 \mathrm{~J}$ \\
\hline C2-Dibenzothiophenes & 0.40 & 1.82 & $3.70 \quad 9.66$ & & $0.40 \mathrm{U}$ & 5.30 \\
\hline C3-Dibenzothiophenes & 0.40 & 1.82 & $0.40 \mathrm{U}$ & $10.7 \quad 0.40$ & $\mathrm{U}$ & 8.92 \\
\hline C4-Dibenzothiophenes & 0.40 & 1.82 & $3.70 \quad 8.50$ & & $0.40 \mathrm{U}$ & 8.25 \\
\hline Fluoranthene & 0.81 & 1.82 & $17.4 \quad 38.0$ & & 47.5 & 45.0 \\
\hline Pyrene & 0.35 & 1.82 & $8.62 \quad 24.9$ & & 25.2 & 29.8 \\
\hline C1-Fluoranthenes/Pyrenes & 0.81 & 1.82 & 11.418 .5 & & 28.7 & 22.9 \\
\hline C2-Fluoranthenes/Pyrenes & 0.81 & 1.82 & 3.239 .05 & & 8.26 & 11.0 \\
\hline C3-Fluoranthenes/Pyrenes & 0.81 & 1.82 & $0.81 \mathrm{U}$ & $0.81 \mathrm{U}$ & $0.81 \mathrm{U}$ & $0.81 \mathrm{U}$ \\
\hline Benzo(a)anthracene & 0.54 & 1.82 & $5.93 \quad 7.10$ & & 17.8 & 14.9 \\
\hline Chrysene & 0.87 & 1.82 & 13.816 .4 & & 40.3 & 31.0 \\
\hline C1-Chrysenes & 0.87 & 1.82 & $3.35 \quad 5.96$ & & 8.38 & 10.1 \\
\hline C2-Chrysenes & 0.87 & 1.82 & $0.87 \mathrm{U}$ & 6.720 .87 & $\mathrm{U}$ & 9.49 \\
\hline C3-Chrysenes & 0.87 & 1.82 & $0.87 \mathrm{U}$ & $0.87 \mathrm{U}$ & $0.87 \mathrm{U}$ & 4.15 \\
\hline C4-Chrysenes & 0.87 & 1.82 & $0.87 \mathrm{U}$ & $0.87 \mathrm{U}$ & $0.87 \mathrm{U}$ & $0.87 \mathrm{U}$ \\
\hline Benzo(b)fluoranthene & 0.37 & 1.82 & 6.428 .35 & & 22.6 & 15.6 \\
\hline Benzo(k)fluoranthene & 0.21 & 1.82 & $2.85 \quad 3.39$ & & 7.76 & 6.60 \\
\hline Benzo(e)pyrene & 0.22 & 1.82 & $3.70 \quad 6.29$ & & 12.8 & 11.1 \\
\hline Benzo(a)pyrene & 0.23 & 1.82 & 2.131 .64 & $\mathrm{~J}$ & 7.69 & 2.97 \\
\hline Perylene & 0.25 & 1.82 & $1.70 \mathrm{~J}$ & $1.06 \mathrm{~J}$ & 3.211 .97 & \\
\hline Indeno(1,2,3-cd)pyrene & 0.14 & 1.82 & $1.64 \mathrm{~J}$ & $1.21 \mathrm{~J}$ & $3.81 \quad 1.90$ & \\
\hline Dibenz(a,h)anthracene & 0.06 & 1.82 & $0.06 \mathrm{U}$ & $0.06 \mathrm{U}$ & $1.47 \mathrm{~J}$ & $0.64 \mathrm{~J}$ \\
\hline Benzo(g,h,i)perylene & 0.22 & 1.82 & $1.21 \mathrm{~J}$ & $1.98 \quad 3.60$ & & 2.89 \\
\hline
\end{tabular}


BATTELLE MARINE SCIENCE LABORATORIES

1529 West Sequim Bay Road

Sequim, Washington 98382-9099

360/681-4564
2010 Regional Mussel Watch - AMB02

ENVVEST 2010

PAHs in Indigenous Mussels UNITS: ng/g wet wt.

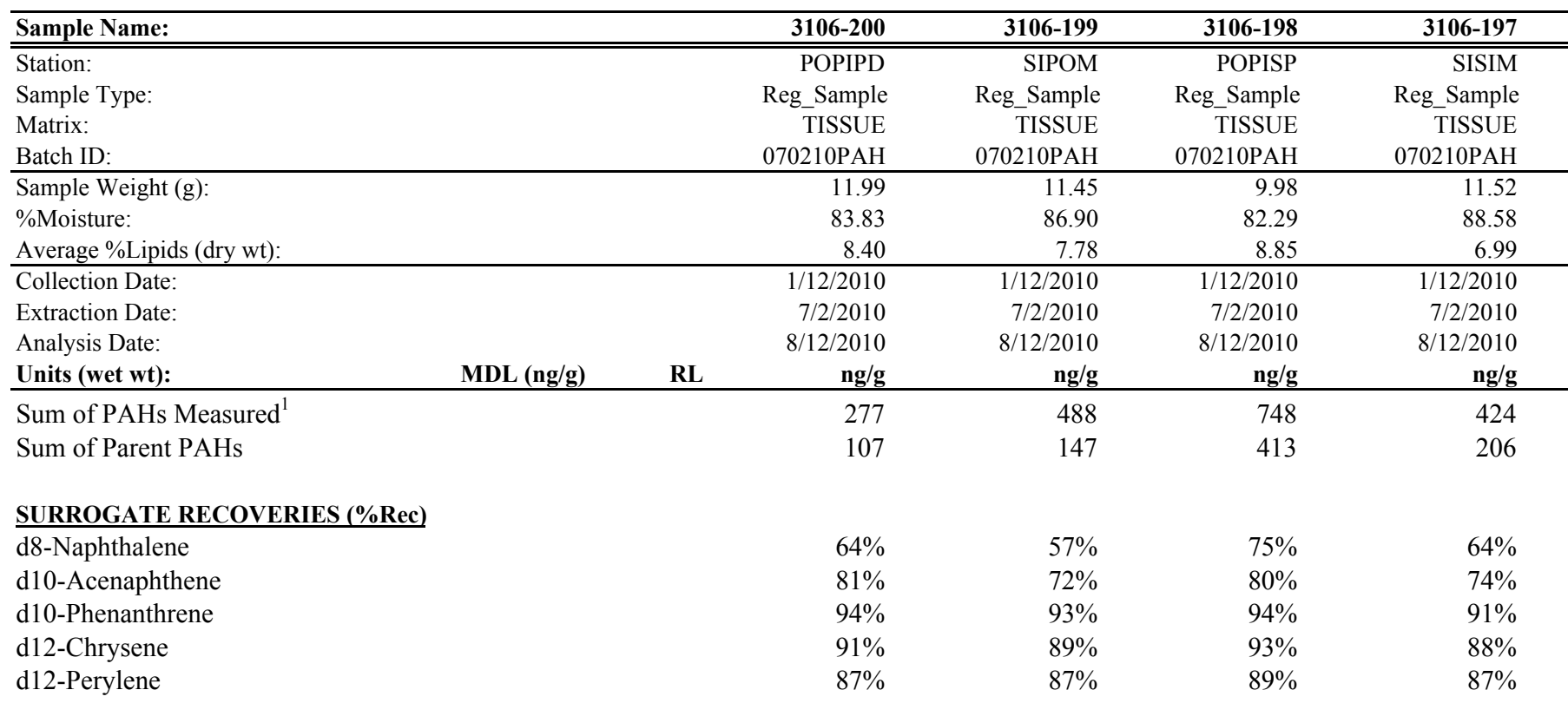

${ }^{1}$ Sum of PAHs includes the MDL for non-detects 
BATTELLE MARINE SCIENCE LABORATORIES

1529 West Sequim Bay Road

Sequim, Washington 98382-9099

360/681-4564
2010 Regional Mussel Watch - AMB02

ENVVEST 2010

PAHs in Indigenous Mussels

UNITS: ng/g wet wt.

\begin{tabular}{|c|c|c|c|c|c|c|}
\hline Sample Name: & & & 3106-191 & 3106-192 & $3106-193$ & 3106-194 \\
\hline Station: & & & PS04 & PS03 & PS06 & PS08 \\
\hline Sample Type: & & & Reg_Sample & Reg_Sample & Reg_Sample & Reg_Sample \\
\hline Matrix: & & & TISSUE & TISSUE & TISSUE & TISSUE \\
\hline Batch ID: & & & 070610PAH & 070610PAH & 070610PAH & 070610PAH \\
\hline Sample Weight (g): & & & 11.59 & 11.49 & 11.06 & 11.29 \\
\hline \%Moisture: & & & 86.49 & 88.23 & 87.82 & 88.33 \\
\hline Average \%Lipids (dry wt): & & & 6.87 & 6.62 & 6.65 & 7.47 \\
\hline Collection Date: & & & $1 / 12 / 2010$ & $1 / 12 / 2010$ & $1 / 12 / 2010$ & $1 / 12 / 2010$ \\
\hline Extraction Date: & & & $7 / 6 / 2010$ & $7 / 6 / 2010$ & $7 / 6 / 2010$ & $7 / 6 / 2010$ \\
\hline Analysis Date: & & & $8 / 19 / 2010$ & $8 / 19 / 2010$ & $8 / 19 / 2010$ & $8 / 19 / 2010$ \\
\hline Units (wet wt): & MDL (ng/g) & $\mathbf{R L}$ & $\mathbf{n g} / \mathrm{g}$ & ng/g & ng/g & $\mathbf{n g} / \mathbf{g}$ \\
\hline Naphthalene & 1.82 & 1.82 & $3.24 \quad 3.70$ & & 3.31 & $7.03 *$ \\
\hline C1-Naphthalenes & 1.82 & 1.82 & 3.173 .32 & & 3.05 & $6.51 *$ \\
\hline C2-Naphthalenes & 3.61 & 1.82 & $6.98 \quad 7.24$ & & 7.01 & 14.6 \\
\hline C3-Naphthalenes & 3.61 & 1.82 & 7.749 .40 & & 7.61 & 15.8 \\
\hline C4-Naphthalenes & 3.61 & 1.82 & $6.84 \quad 7.77$ & & 4.92 & 20.9 \\
\hline Biphenyl & 2.86 & 1.82 & $2.86 \mathrm{U}$ & $2.86 \mathrm{U}$ & $2.86 \mathrm{U}$ & $2.86 \mathrm{U}$ \\
\hline Acenaphthylene & 1.68 & 1.82 & 1.881 .68 & $\mathrm{U}$ & $1.68 \mathrm{U}$ & 3.36 \\
\hline Acenaphthene & 2.29 & 1.82 & $2.29 \mathrm{U}$ & $2.29 \mathrm{U}$ & $2.29 \mathrm{U}$ & 2.32 \\
\hline Fluorene & 2.75 & 1.82 & $2.75 \mathrm{U}$ & $3.14 \quad 2.75$ & $\mathrm{U}$ & 3.83 \\
\hline C1-Fluorenes & 2.17 & 1.82 & 13.117 .1 & & 14.9 & 20.6 \\
\hline C2-Fluorenes & 2.17 & 1.82 & $2.17 \mathrm{U}$ & $2.17 \mathrm{U}$ & $2.17 \mathrm{U}$ & $2.17 \mathrm{U}$ \\
\hline C3-Fluorenes & 2.17 & 1.82 & $2.17 \mathrm{U}$ & $2.17 \mathrm{U}$ & $2.17 \mathrm{U}$ & $2.17 \mathrm{U}$ \\
\hline Anthracene & 0.42 & 1.82 & $5.73 \quad 8.14$ & & 4.31 & 6.75 \\
\hline Phenanthrene & 1.10 & 1.82 & 20.723 .2 & & 14.0 & 32.5 \\
\hline C1-Phenanthrenes/Anthracenes & 1.18 & 1.82 & 21.823 .3 & & 18.9 & 54.8 \\
\hline C2-Phenanthrenes/Anthracenes & 0.10 & 1.82 & $23.0 \quad 29.3$ & & 19.7 & 64.4 \\
\hline C3-Phenanthrenes/Anthracenes & 0.10 & 1.82 & $15.6 \quad 22.3$ & & 15.3 & 68.7 \\
\hline C4-Phenanthrenes/Anthracenes & 0.10 & 1.82 & $0.10 \mathrm{U}$ & $0.10 \mathrm{U}$ & $0.10 \mathrm{U}$ & $0.10 \mathrm{U}$ \\
\hline Dibenzothiophene & 0.40 & 1.82 & $0.79 \mathrm{~J}$ & $1.04 \mathrm{~J}$ & $0.71 \mathrm{~J}$ & $1.53 \mathrm{~J}$ \\
\hline C1-Dibenzothiophenes & 0.40 & 1.82 & $1.35 \mathrm{~J}$ & $1.78 \mathrm{~J}$ & $1.55 \mathrm{~J}$ & 3.86 \\
\hline C2-Dibenzothiophenes & 0.40 & 1.82 & 4.565 .63 & & 5.48 & 13.6 \\
\hline C3-Dibenzothiophenes & 0.40 & 1.82 & 6.669 .36 & & 5.71 & 12.5 \\
\hline C4-Dibenzothiophenes & 0.40 & 1.82 & 6.8912 .9 & & 8.23 & 10.6 \\
\hline Fluoranthene & 0.81 & 1.82 & $48.1 \quad 52.7$ & & 26.0 & 78.1 \\
\hline Pyrene & 0.35 & 1.82 & $26.6 \quad 32.8$ & & 17.6 & 47.9 \\
\hline C1-Fluoranthenes/Pyrenes & 0.81 & 1.82 & 22.428 .2 & & 14.7 & 33.0 \\
\hline C2-Fluoranthenes/Pyrenes & 0.81 & 1.82 & $9.29 \quad 15.4$ & & 7.36 & 17.7 \\
\hline C3-Fluoranthenes/Pyrenes & 0.81 & 1.82 & $0.81 \mathrm{U}$ & 12.10 .81 & $\mathrm{U}$ & 10.4 \\
\hline Benzo(a)anthracene & 0.54 & 1.82 & 19.323 .8 & & 12.1 & 26.8 \\
\hline Chrysene & 0.87 & 1.82 & 29.135 .2 & & 19.6 & 46.3 \\
\hline C1-Chrysenes & 0.87 & 1.82 & 8.9614 .4 & & 7.14 & 14.6 \\
\hline C2-Chrysenes & 0.87 & 1.82 & 7.6413 .3 & & 7.19 & 11.5 \\
\hline C3-Chrysenes & 0.87 & 1.82 & $0.87 \mathrm{U}$ & $0.87 \mathrm{U}$ & $0.87 \mathrm{U}$ & $0.87 \mathrm{U}$ \\
\hline C4-Chrysenes & 0.87 & 1.82 & $0.87 \mathrm{U}$ & $0.87 \mathrm{U}$ & $0.87 \mathrm{U}$ & $0.87 \mathrm{U}$ \\
\hline Benzo(b)fluoranthene & 0.37 & 1.82 & 15.725 .7 & & 10.7 & 24.3 \\
\hline Benzo(k)fluoranthene & 0.21 & 1.82 & 7.5410 .9 & & 4.96 & 11.0 \\
\hline Benzo(e)pyrene & 0.22 & 1.82 & 9.7916 .0 & & 6.71 & 14.4 \\
\hline Benzo(a)pyrene & 0.23 & 1.82 & 4.717 .80 & & 3.53 & 5.93 \\
\hline Perylene & 0.25 & 1.82 & 1.822 .86 & & $1.32 \mathrm{~J}$ & 2.35 \\
\hline Indeno(1,2,3-cd)pyrene & 0.14 & 1.82 & 2.410 .87 & $\mathrm{~J}$ & 2.68 & 4.25 \\
\hline Dibenz(a,h)anthracene & 0.06 & 1.82 & $0.06 \mathrm{U}$ & $0.06 \mathrm{U}$ & $0.06 \mathrm{U}$ & $0.06 \mathrm{U}$ \\
\hline $\operatorname{Benzo}(\mathrm{g}, \mathrm{h}, \mathrm{i})$ perylene & 0.22 & 1.82 & 1.930 .92 & $\mathrm{~J}$ & 6.00 & 3.99 \\
\hline
\end{tabular}


BATTELLE MARINE SCIENCE LABORATORIES

1529 West Sequim Bay Road

Sequim, Washington 98382-9099

360/681-4564
2010 Regional Mussel Watch - AMB02

ENVVEST 2010

PAHs in Indigenous Mussels

UNITS: ng/g wet wt.

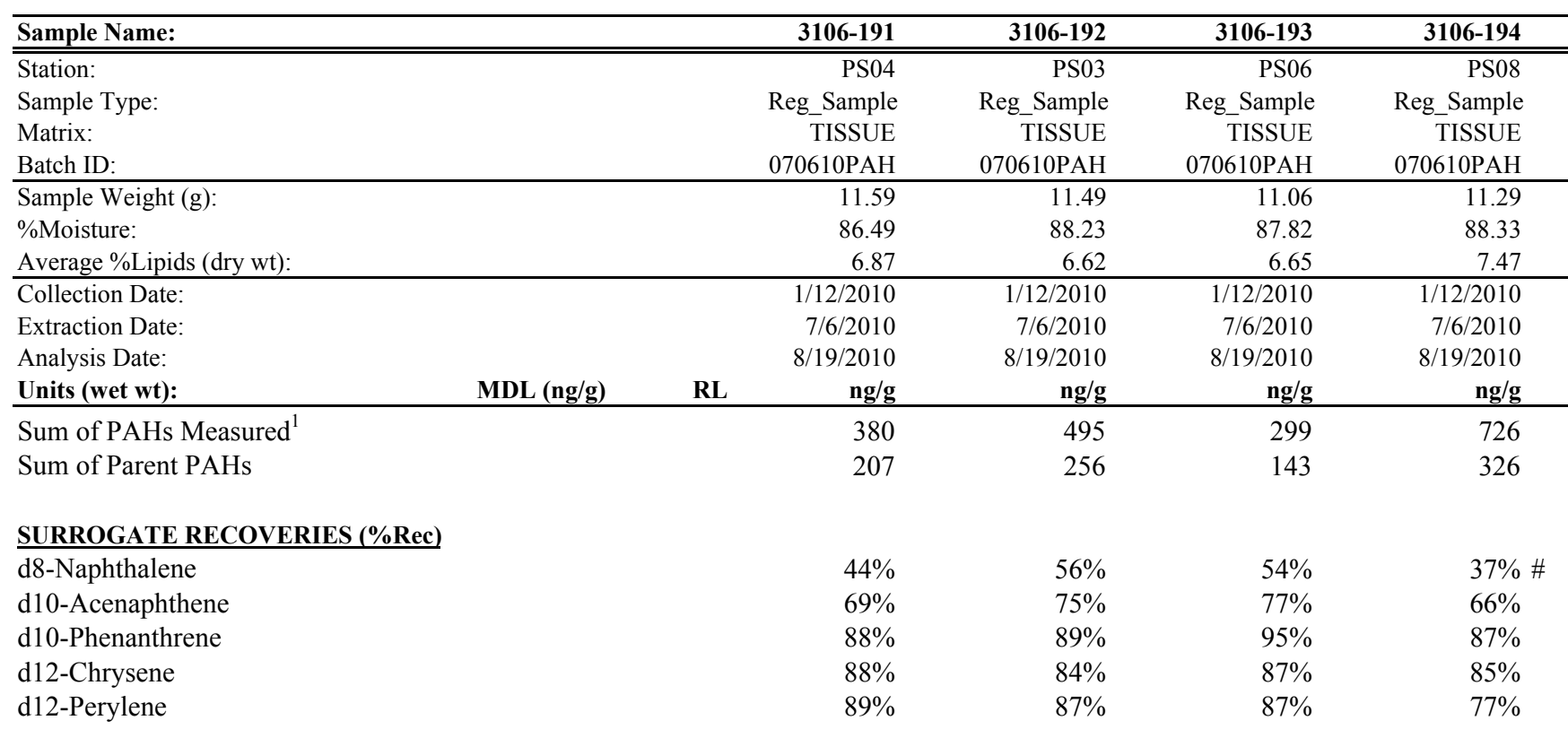

${ }^{1}$ Sum of PAHs includes the MDL for non-detects 
BATTELLE MARINE SCIENCE LABORATORIES

1529 West Sequim Bay Road

Sequim, Washington 98382-9099

360/681-4564
2010 Regional Mussel Watch - AMB02

ENVVEST 2010

PAHs in Indigenous Mussels

UNITS: ng/g wet wt.

\begin{tabular}{|c|c|c|c|c|c|c|c|c|}
\hline Sample Name: & & & 3106-195 & & 3106-196 & & $3106-201$ & $3106-202$ \\
\hline Station: & & & $\overline{\text { PS09 }}$ & & PS11 & & PWNLP & DYOTS \\
\hline Sample Type: & & & Reg_Sample & & Reg_Sample & & Reg_Sample & Reg_Sample \\
\hline Matrix: & & & TISSUE & & TISSUE & & TISSUE & TISSUE \\
\hline Batch ID: & & & 070610PAH & & 070610PAH & & 071910PAH & 071910PAH \\
\hline Sample Weight (g): & & & 11.86 & & 12.46 & & 11.4 & 13.26 \\
\hline \%Moisture: & & & 88.74 & & 87.88 & & 83.92 & 83.37 \\
\hline Average \%Lipids (dry wt): & & & 6.24 & & 6.96 & & 9.40 & 8.61 \\
\hline Collection Date: & & & $1 / 12 / 2010$ & & $1 / 12 / 2010$ & & $1 / 12 / 2010$ & $1 / 12 / 2010$ \\
\hline Extraction Date: & & & $7 / 6 / 2010$ & & $7 / 6 / 2010$ & & $7 / 19 / 2010$ & $7 / 19 / 2010$ \\
\hline Analysis Date: & & & $8 / 19 / 2010$ & & $8 / 19 / 2010$ & & $8 / 13 / 2010$ & $8 / 13 / 2010$ \\
\hline Units (wet wt): & MDL (ng/g) & RL & $\mathrm{ng} / \mathrm{g}$ & & ng/g & & $\mathbf{n g} / \mathrm{g}$ & $\mathrm{ng} / \mathrm{g}$ \\
\hline Naphthalene & 1.82 & 1.82 & 5.85 & 41.7 & & & $14.0 \mathrm{~B}^{*}$ & $6.99 \mathrm{~B}^{*}$ \\
\hline C1-Naphthalenes & 1.82 & 1.82 & 4.81 & 30.9 & & & $8.16 *$ & $4.61 *$ \\
\hline C2-Naphthalenes & 3.61 & 1.82 & 7.88 & & 36.0 & 10.9 & 9.32 & \\
\hline C3-Naphthalenes & 3.61 & 1.82 & 7.48 & & 26.8 & 11.1 & 10.8 & \\
\hline C4-Naphthalenes & 3.61 & 1.82 & 6.95 & & 13.1 & 7.21 & 8.44 & \\
\hline Biphenyl & 2.86 & 1.82 & 2.86 & & 6.56 & 2.86 & $\mathrm{U}$ & $2.86 \mathrm{U}$ \\
\hline Acenaphthylene & 1.68 & 1.82 & 2.15 & 5.71 & & & $1.68 \mathrm{U}$ & 2.50 \\
\hline Acenaphthene & 2.29 & 1.82 & 2.45 & 37.1 & & & $2.29 \mathrm{U}$ & 2.84 \\
\hline Fluorene & 2.75 & 1.82 & 3.21 & 46.5 & & & $2.75 \mathrm{U}$ & 3.11 \\
\hline C1-Fluorenes & 2.17 & 1.82 & 14.7 & & 25.6 & 28.0 & 31.9 & \\
\hline C2-Fluorenes & 2.17 & 1.82 & 2.17 & & 2.17 & $\mathrm{U}$ & $2.17 \mathrm{U}$ & $2.17 \mathrm{U}$ \\
\hline C3-Fluorenes & 2.17 & 1.82 & 2.17 & $\mathrm{U}$ & 2.17 & $\mathrm{U}$ & $2.17 \mathrm{U}$ & $2.17 \mathrm{U}$ \\
\hline Anthracene & 0.42 & 1.82 & 5.31 & & 22.8 & 2.56 & 4.90 & \\
\hline Phenanthrene & 1.10 & 1.82 & 30.9 & & 516.2 & 6.50 & 16.2 & \\
\hline C1-Phenanthrenes/Anthracenes & 1.18 & 1.82 & 22.2 & & 184.7 & 9.14 & 18.1 & \\
\hline C2-Phenanthrenes/Anthracenes & 0.10 & 1.82 & 23.6 & & 92.5 & 11.8 & 17.3 & \\
\hline C3-Phenanthrenes/Anthracenes & 0.10 & 1.82 & 17.6 & & 39.3 & 12.9 & 23.1 & \\
\hline C4-Phenanthrenes/Anthracenes & 0.10 & 1.82 & 0.10 & & 0.10 & $\mathrm{U}$ & $0.10 \mathrm{U}$ & $0.10 \mathrm{U}$ \\
\hline Dibenzothiophene & 0.40 & 1.82 & 1.43 & & 27.1 & 0.40 & $\mathrm{U}$ & $0.61 \mathrm{~J}$ \\
\hline C1-Dibenzothiophenes & 0.40 & 1.82 & 1.72 & & 12.1 & 1.24 & $\mathrm{~J}$ & $1.43 \mathrm{~J}$ \\
\hline C2-Dibenzothiophenes & 0.40 & 1.82 & 5.82 & & 11.1 & 4.27 & 5.69 & \\
\hline C3-Dibenzothiophenes & 0.40 & 1.82 & 8.56 & & 8.76 & 5.60 & 5.21 & \\
\hline C4-Dibenzothiophenes & 0.40 & 1.82 & 8.60 & & 9.16 & 4.42 & 7.10 & \\
\hline Fluoranthene & 0.81 & 1.82 & 46.2 & & 497.3 & 8.66 & 26.2 & \\
\hline Pyrene & 0.35 & 1.82 & 28.3 & & 299.3 & 5.87 & 17.0 & \\
\hline C1-Fluoranthenes/Pyrenes & 0.81 & 1.82 & 18.6 & & 96.4 & 6.61 & 13.4 & \\
\hline C2-Fluoranthenes/Pyrenes & 0.81 & 1.82 & 14.5 & & 131.6 & 3.90 & 5.68 & \\
\hline C3-Fluoranthenes/Pyrenes & 0.81 & 1.82 & 8.90 & 33.1 & & & $0.81 \mathrm{U}$ & $0.81 \mathrm{U}$ \\
\hline Benzo(a)anthracene & 0.54 & 1.82 & 20.0 & & 50.3 & 3.49 & 9.66 & \\
\hline Chrysene & 0.87 & 1.82 & 31.3 & & 118.1 & 7.85 & 15.6 & \\
\hline C1-Chrysenes & 0.87 & 1.82 & 9.10 & & 25.2 & 3.88 & 6.02 & \\
\hline C2-Chrysenes & 0.87 & 1.82 & 9.18 & & 16.7 & 6.61 & 7.79 & \\
\hline C3-Chrysenes & 0.87 & 1.82 & 0.87 & $\mathrm{U}$ & 0.87 & $\mathrm{U}$ & $0.87 \mathrm{U}$ & $0.87 \mathrm{U}$ \\
\hline C4-Chrysenes & 0.87 & 1.82 & 0.87 & $\mathrm{U}$ & 0.87 & $\mathrm{U}$ & $0.87 \mathrm{U}$ & $0.87 \mathrm{U}$ \\
\hline Benzo(b)fluoranthene & 0.37 & 1.82 & 18.3 & & 58.0 & 5.12 & 9.26 & \\
\hline Benzo(k)fluoranthene & 0.21 & 1.82 & 7.32 & & 22.0 & 2.04 & 3.59 & \\
\hline Benzo(e)pyrene & 0.22 & 1.82 & 10.6 & & 27.5 & 3.42 & 7.06 & \\
\hline Benzo(a)pyrene & 0.23 & 1.82 & 5.92 & 14.0 & & & $1.60 \mathrm{~J}$ & 2.18 \\
\hline Perylene & 0.25 & 1.82 & 2.03 & 3.75 & & & $1.31 \mathrm{~J}$ & 1.96 \\
\hline Indeno(1,2,3-cd)pyrene & 0.14 & 1.82 & 1.61 & & 2.19 & 1.78 & $\mathrm{~J}$ & $1.33 \mathrm{~J}$ \\
\hline Dibenz(a,h)anthracene & 0.06 & 1.82 & 0.06 & & 3.11 & 0.06 & $\mathrm{U}$ & $0.06 \mathrm{U}$ \\
\hline Benzo(g,h,i)perylene & 0.22 & 1.82 & 0.74 & & 4.32 & 2.27 & 2.04 & \\
\hline
\end{tabular}


BATTELLE MARINE SCIENCE LABORATORIES

1529 West Sequim Bay Road

Sequim, Washington 98382-9099

360/681-4564
2010 Regional Mussel Watch - AMB02

ENVVEST 2010

PAHs in Indigenous Mussels

UNITS: ng/g wet wt.

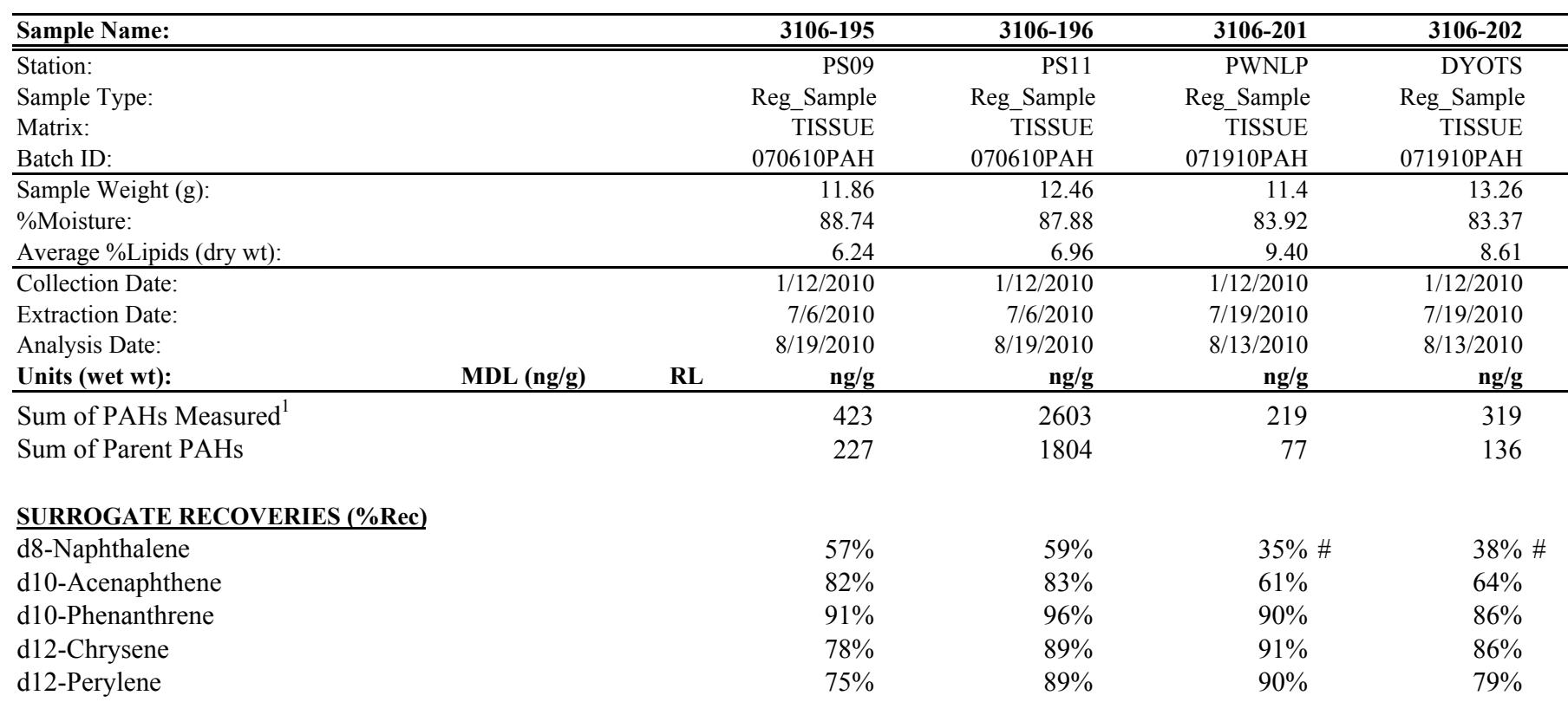

${ }^{1}$ Sum of PAHs includes the MDL for non-detects 
BATTELLE MARINE SCIENCE LABORATORIES

1529 West Sequim Bay Road

Sequim, Washington 98382-9099

360/681-4564
2010 Regional Mussel Watch - AMB02

ENVVEST 2010

PAHs in Indigenous Mussels

UNITS: ng/g wet wt.

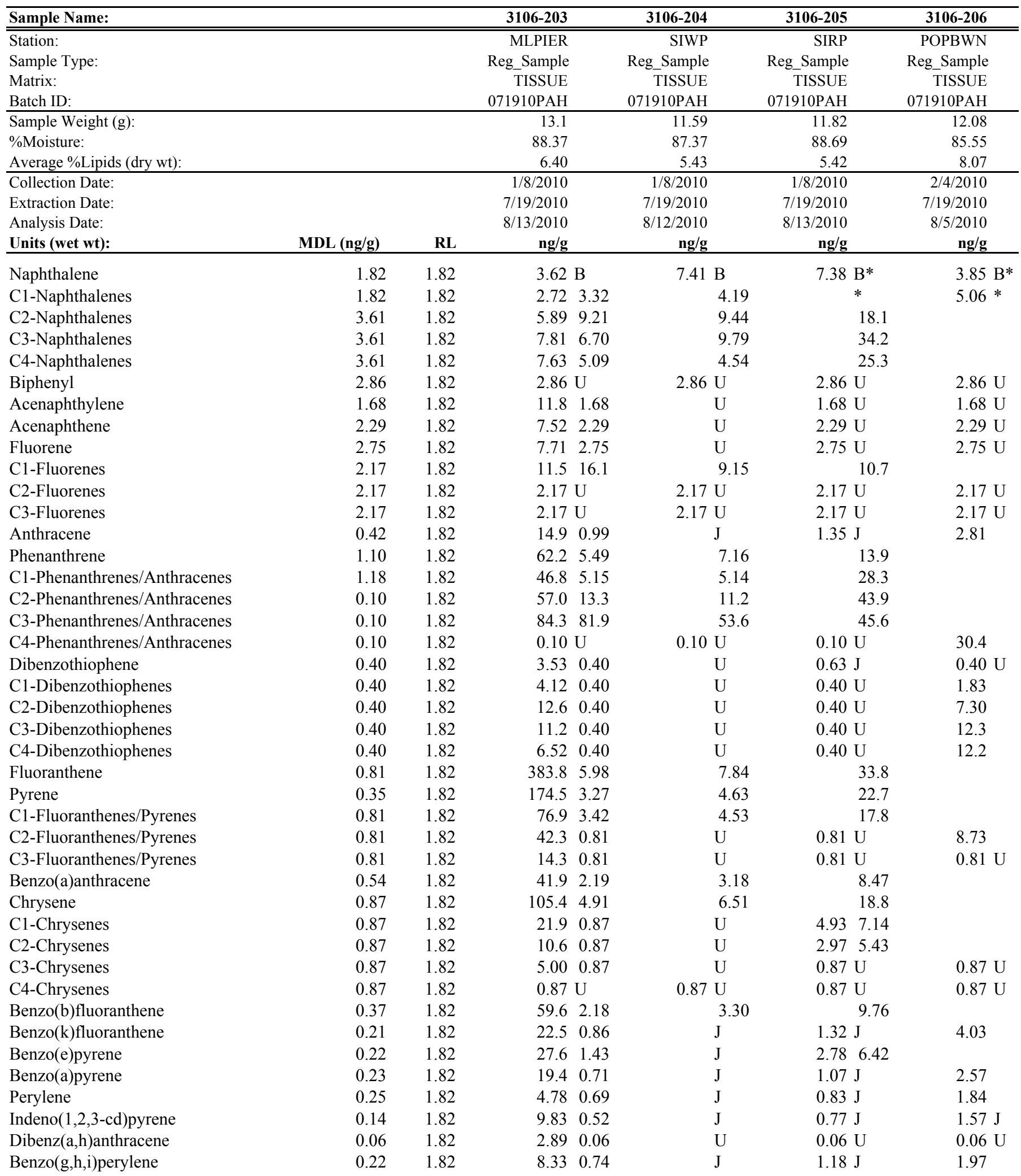


BATTELLE MARINE SCIENCE LABORATORIES

1529 West Sequim Bay Road

Sequim, Washington 98382-9099

360/681-4564
2010 Regional Mussel Watch - AMB02

ENVVEST 2010

PAHs in Indigenous Mussels

UNITS: ng/g wet wt.

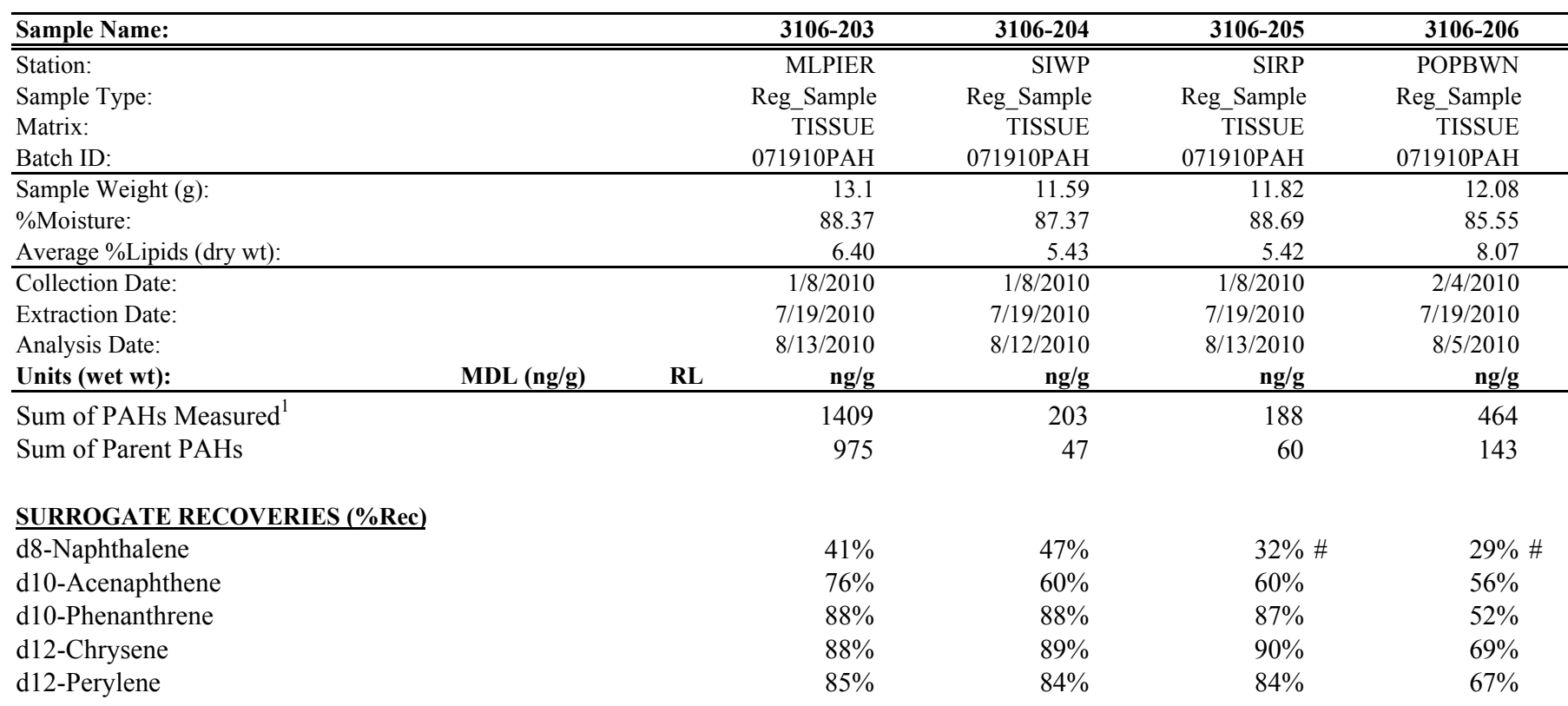

${ }^{1}$ Sum of PAHs includes the MDL for non-detects 
BATTELLE MARINE SCIENCE LABORATORIES

1529 West Sequim Bay Road

Sequim, Washington 98382-9099

360/681-4564
2010 Regional Mussel Watch - AMB02

ENVVEST 2010

PAHs in Indigenous Mussels

UNITS: ng/g wet wt.

\begin{tabular}{|c|c|c|c|c|c|c|c|c|}
\hline Sample Name: & & & $3107-207$ & $3106-208$ & 3106-209 & & $3106-210$ & \\
\hline Station: & & & KPTPIER & $\overline{\text { KPTLAG }}$ & APHCB & & APKIANA & \\
\hline Sample Type: & & & Reg_Sample & Reg_Sample & Reg_Sample & & Reg_Sample & \\
\hline Matrix: & & & TISSUE & TISSUE & TISSUE & & TISSUE & \\
\hline Batch ID: & & & 071910PAH & 071910РAH & 071910PAH & & 072910PAH & \\
\hline Sample Weight (g): & & & 11.62 & 12.8 & 11.6 & & 11.63 & \\
\hline \%Moisture: & & & 83.65 & 87.99 & 84.88 & & 83.12 & \\
\hline Average \%Lipids (dry wt): & & & 7.92 & 7.55 & 5.52 & & 7.57 & \\
\hline Collection Date: & & & $2 / 4 / 2010$ & $2 / 4 / 2010$ & $2 / 4 / 2010$ & & $2 / 4 / 2010$ & \\
\hline Extraction Date: & & & $7 / 19 / 2010$ & $7 / 19 / 2010$ & $7 / 19 / 2010$ & & $7 / 29 / 2010$ & \\
\hline Analysis Date: & & & $8 / 5 / 2010$ & $8 / 5 / 2010$ & $8 / 8 / 2010$ & & $8 / 7 / 2010$ & \\
\hline Units (wet wt): & MDL (ng/g) & $\mathbf{R L}$ & ng/g & ng/g & ng/g & & $\mathrm{ng} / \mathrm{g}$ & \\
\hline Naphthalene & 1.82 & 1.82 & $3.86 \mathrm{~B}$ & $4.33 \mathrm{~B}$ & 17.2 & B & 2.77 & \\
\hline C1-Naphthalenes & 1.82 & 1.82 & $3.68 \quad 3.11$ & 18.4 & & 1.86 & & \\
\hline C2-Naphthalenes & 3.61 & 1.82 & $21.0 \quad 12.4$ & 50.9 & & 10.7 & & \\
\hline C3-Naphthalenes & 3.61 & 1.82 & 11.64 .65 & 33.4 & & 9.92 & & \\
\hline C4-Naphthalenes & 3.61 & 1.82 & 18.23 .61 & $\mathrm{U}$ & 12.3 & 5.66 & & \\
\hline Biphenyl & 2.86 & 1.82 & $2.86 \mathrm{U}$ & $2.86 \mathrm{U}$ & 5.51 & 2.86 & & $\mathrm{U}$ \\
\hline Acenaphthylene & 1.68 & 1.82 & $1.97 \quad 1.68$ & $\mathrm{U}$ & 4.06 & 1.68 & & $\mathrm{U}$ \\
\hline Acenaphthene & 2.29 & 1.82 & $2.29 \mathrm{U}$ & $2.29 \mathrm{U}$ & 69.0 & 2.29 & & $\mathrm{U}$ \\
\hline Fluorene & 2.75 & 1.82 & $2.75 \mathrm{U}$ & $2.75 \mathrm{U}$ & 63.4 & 2.75 & & $\mathrm{U}$ \\
\hline C1-Fluorenes & 2.17 & 1.82 & 14.17 .73 & 24.3 & & 9.33 & & \\
\hline C2-Fluorenes & 2.17 & 1.82 & $2.17 \mathrm{U}$ & $2.17 \mathrm{U}$ & 2.17 & $\mathrm{U}$ & 2.17 & $\mathrm{U}$ \\
\hline C3-Fluorenes & 2.17 & 1.82 & $2.17 \mathrm{U}$ & $2.17 \mathrm{U}$ & 2.17 & $\mathrm{U}$ & 2.17 & $\mathrm{U}$ \\
\hline Anthracene & 0.42 & 1.82 & $3.24 \quad 2.18$ & 45.1 & & 1.77 & & $\mathrm{~J}$ \\
\hline Phenanthrene & 1.10 & 1.82 & $8.70 \quad 8.87$ & & 141.8 & 6.96 & & \\
\hline C1-Phenanthrenes/Anthracenes & 1.18 & 1.82 & 16.58 .75 & 59.7 & & 9.89 & & \\
\hline C2-Phenanthrenes/Anthracenes & 0.10 & 1.82 & 31.48 .10 & 27.2 & & 8.77 & & \\
\hline C3-Phenanthrenes/Anthracenes & 0.10 & 1.82 & 35.414 .5 & 13.4 & & 13.4 & & \\
\hline C4-Phenanthrenes/Anthracenes & 0.10 & 1.82 & $0.10 \mathrm{U}$ & $0.10 \mathrm{U}$ & 0.10 & $\mathrm{U}$ & 0.10 & $\mathrm{U}$ \\
\hline Dibenzothiophene & 0.40 & 1.82 & $0.40 \mathrm{U}$ & $0.40 \mathrm{U}$ & 9.00 & 0.40 & & $\mathrm{U}$ \\
\hline C1-Dibenzothiophenes & 0.40 & 1.82 & 4.760 .85 & $\mathrm{~J}$ & 4.35 & 0.87 & & $\mathrm{~J}$ \\
\hline C2-Dibenzothiophenes & 0.40 & 1.82 & 20.43 .57 & 5.49 & & 0.40 & & $\mathrm{U}$ \\
\hline C3-Dibenzothiophenes & 0.40 & 1.82 & 21.12 .94 & 3.85 & & 2.59 & & \\
\hline C4-Dibenzothiophenes & 0.40 & 1.82 & $14.7 \quad 0.40$ & $\mathrm{U}$ & 0.40 & $\mathrm{U}$ & 2.86 & \\
\hline Fluoranthene & 0.81 & 1.82 & 17.214 .4 & & 131 & 11.7 & & \\
\hline Pyrene & 0.35 & 1.82 & 10.410 .3 & 81.5 & & 5.74 & & \\
\hline C1-Fluoranthenes/Pyrenes & 0.81 & 1.82 & 9.118 .13 & 73.1 & & 6.59 & & \\
\hline C2-Fluoranthenes/Pyrenes & 0.81 & 1.82 & 4.863 .85 & 15.5 & & 2.67 & & \\
\hline C3-Fluoranthenes/Pyrenes & 0.81 & 1.82 & $0.81 \mathrm{U}$ & $0.81 \mathrm{U}$ & 0.81 & $\mathrm{U}$ & 0.81 & $\mathrm{U}$ \\
\hline Benzo(a)anthracene & 0.54 & 1.82 & 4.126 .59 & 64.1 & & 4.79 & & \\
\hline Chrysene & 0.87 & 1.82 & 7.798 .67 & & 118 & 8.63 & & \\
\hline C1-Chrysenes & 0.87 & 1.82 & $4.12 \quad 3.34$ & 16.3 & & 3.16 & & \\
\hline C2-Chrysenes & 0.87 & 1.82 & $5.40 \quad 4.71$ & 6.76 & & 3.83 & & \\
\hline C3-Chrysenes & 0.87 & 1.82 & $0.87 \mathrm{U}$ & $0.87 \mathrm{U}$ & 0.87 & $\mathrm{U}$ & 0.87 & $\mathrm{U}$ \\
\hline C4-Chrysenes & 0.87 & 1.82 & $0.87 \mathrm{U}$ & $0.87 \mathrm{U}$ & 0.87 & $\mathrm{U}$ & 0.87 & $\mathrm{U}$ \\
\hline Benzo(b)fluoranthene & 0.37 & 1.82 & 4.924 .59 & 27.3 & & 4.26 & & \\
\hline Benzo(k)fluoranthene & 0.21 & 1.82 & 1.871 .39 & $\mathrm{~J}$ & 11.0 & 1.68 & & $\mathrm{~J}$ \\
\hline Benzo(e)pyrene & 0.22 & 1.82 & 3.962 .94 & 12.9 & & 3.11 & & \\
\hline Benzo(a)pyrene & 0.23 & 1.82 & $1.52 \mathrm{~J}$ & $2.15 \quad 14.7$ & & 1.99 & & \\
\hline Perylene & 0.25 & 1.82 & $1.16 \mathrm{~J}$ & $1.17 \mathrm{~J}$ & 4.24 & 0.88 & & $\mathrm{~J}$ \\
\hline Indeno(1,2,3-cd)pyrene & 0.14 & 1.82 & $1.01 \mathrm{~J}$ & $0.85 \mathrm{~J}$ & 5.97 & 0.72 & & $\mathrm{~J}$ \\
\hline Dibenz(a,h)anthracene & 0.06 & 1.82 & $0.06 \mathrm{U}$ & $0.06 \mathrm{U}$ & 2.12 & 0.06 & & $\mathrm{U}$ \\
\hline Benzo(g,h,i)perylene & 0.22 & 1.82 & $1.07 \mathrm{~J}$ & $0.92 \mathrm{~J}$ & 4.47 & 0.82 & & $\mathrm{~J}$ \\
\hline
\end{tabular}


BATTELLE MARINE SCIENCE LABORATORIES

1529 West Sequim Bay Road

Sequim, Washington 98382-9099

360/681-4564
2010 Regional Mussel Watch - AMB02

ENVVEST 2010

PAHs in Indigenous Mussels UNITS: ng/g wet wt.

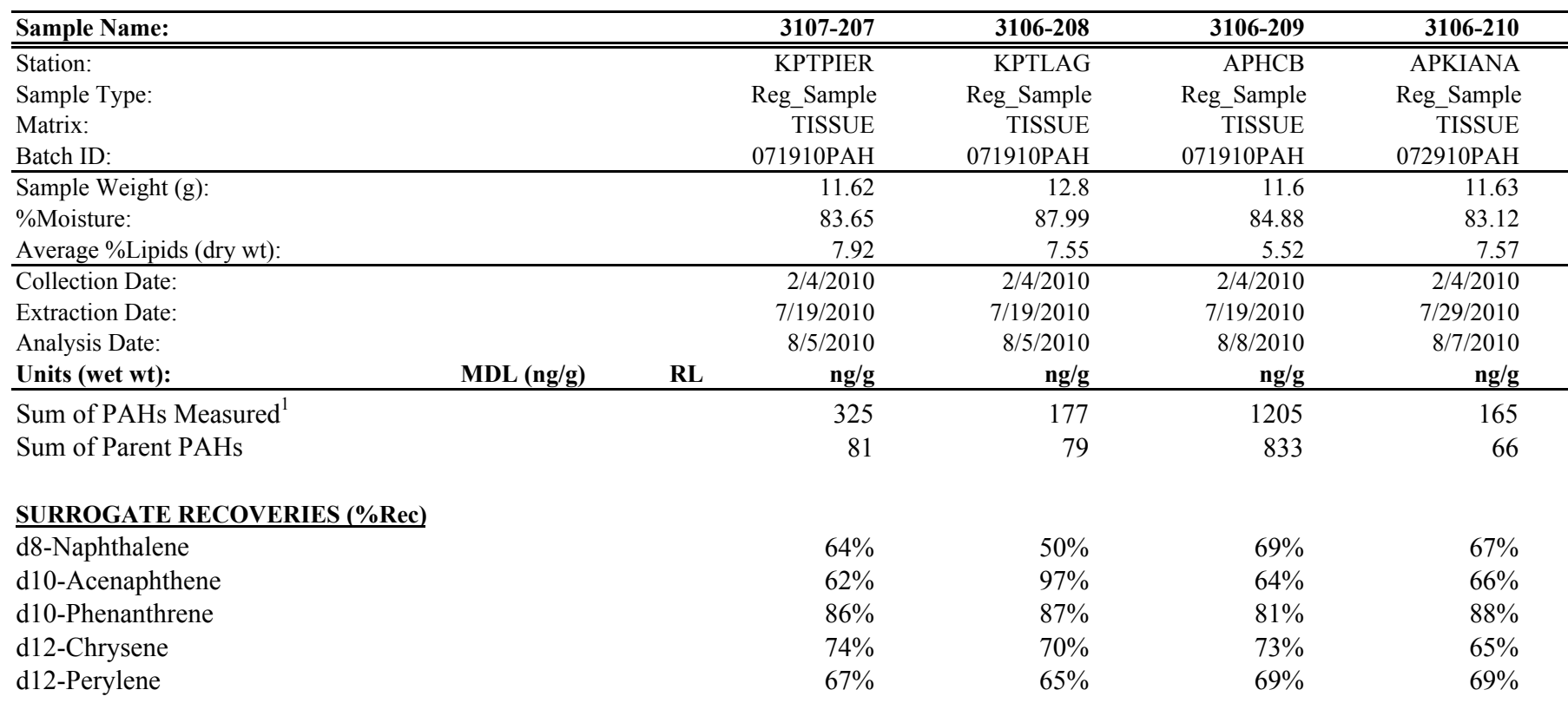

${ }^{1}$ Sum of PAHs includes the MDL for non-detects 
BATTELLE MARINE SCIENCE LABORATORIES

1529 West Sequim Bay Road

Sequim, Washington 98382-9099

360/681-4564
2010 Regional Mussel Watch - AMB02

ENVVEST 2010

PAHs in Indigenous Mussels

UNITS: ng/g wet wt.

\begin{tabular}{|c|c|c|c|c|c|c|c|}
\hline Sample Name: & & & $3106-211$ & 3106-212 & $3106-213$ & & $3106-214$ \\
\hline Station: & & & LBPMSC & DYOBAP & SIGST & & PS01 \\
\hline Sample Type: & & & Reg_Sample & Reg_Sample & Reg_Sample & & Reg_Sample \\
\hline Matrix: & & & TISSUE & TISSUE & TISSUE & & TISSUE \\
\hline Batch ID: & & & 072910РAH & 072910PAH & 072910РAH & & 072910РAH \\
\hline Sample Weight (g): & & & 11.45 & 12.05 & 12.31 & & 13.03 \\
\hline \%Moisture: & & & 83.49 & 83.51 & 84.23 & & 84.79 \\
\hline Average \%Lipids (dry wt): & & & 7.02 & 7.97 & 9.08 & & 8.63 \\
\hline Collection Date: & & & $2 / 4 / 2010$ & $2 / 3 / 2010$ & $2 / 3 / 2010$ & & $2 / 2 / 2010$ \\
\hline Extraction Date: & & & $7 / 29 / 2010$ & $7 / 29 / 2010$ & $7 / 29 / 2010$ & & $7 / 29 / 2010$ \\
\hline Analysis Date: & & & $8 / 7 / 2010$ & $8 / 7 / 2010$ & $8 / 7 / 2010$ & & $8 / 7 / 2010$ \\
\hline Units (wet wt): & MDL (ng/g) & $\mathbf{R L}$ & ng/g & ng/g & ng/g & & ng/g \\
\hline Naphthalene & 1.82 & 1.82 & $3.52 \mathrm{~B}$ & $2.23 \mathrm{~B}$ & $3.80 \mathrm{~B}$ & & $3.17 \mathrm{~B}$ \\
\hline C1-Naphthalenes & 1.82 & 1.82 & $5.48 \quad 1.98$ & 2.66 & & 2.47 & \\
\hline C2-Naphthalenes & 3.61 & 1.82 & $14.0 \quad 8.87$ & 17.8 & & 14.2 & \\
\hline C3-Naphthalenes & 3.61 & 1.82 & $22.5 \quad 9.91$ & 14.7 & & 10.6 & \\
\hline C4-Naphthalenes & 3.61 & 1.82 & 29.73 .75 & 8.21 & & 6.33 & \\
\hline Biphenyl & 2.86 & 1.82 & $2.86 \mathrm{U}$ & $2.86 \mathrm{U}$ & $2.86 \mathrm{~L}$ & & $2.86 \mathrm{U}$ \\
\hline Acenaphthylene & 1.68 & 1.82 & $2.34 \quad 1.68$ & $\mathrm{U}$ & $1.80 \mathrm{~J}$ & & $1.68 \mathrm{U}$ \\
\hline Acenaphthene & 2.29 & 1.82 & $2.29 \mathrm{U}$ & $2.29 \mathrm{U}$ & $2.29 \mathrm{~L}$ & & $2.29 \mathrm{U}$ \\
\hline Fluorene & 2.75 & 1.82 & $2.75 \mathrm{U}$ & $2.75 \mathrm{U}$ & $2.75 \mathrm{~L}$ & & $2.75 \mathrm{U}$ \\
\hline C1-Fluorenes & 2.17 & 1.82 & 12.111 .6 & 14.1 & & 9.91 & \\
\hline C2-Fluorenes & 2.17 & 1.82 & $2.17 \mathrm{U}$ & $2.17 \mathrm{U}$ & $2.17 \mathrm{~L}$ & & $2.17 \mathrm{U}$ \\
\hline C3-Fluorenes & 2.17 & 1.82 & $2.17 \mathrm{U}$ & $2.17 \mathrm{U}$ & $2.17 \mathrm{~L}$ & & $2.17 \mathrm{U}$ \\
\hline Anthracene & 0.42 & 1.82 & $4.98 \quad 1.60$ & $\mathrm{~J}$ & 2.65 & 2.41 & \\
\hline Phenanthrene & 1.10 & 1.82 & $27.4 \quad 4.28$ & 6.47 & & 6.07 & \\
\hline C1-Phenanthrenes/Anthracenes & 1.18 & 1.82 & $45.4 \quad 10.2$ & 17.8 & & 13.7 & \\
\hline C2-Phenanthrenes/Anthracenes & 0.10 & 1.82 & 61.97 .58 & 14.4 & & 14.0 & \\
\hline C3-Phenanthrenes/Anthracenes & 0.10 & 1.82 & 51.914 .6 & 95.3 & & 27.7 & \\
\hline C4-Phenanthrenes/Anthracenes & 0.10 & 1.82 & $46.0 \quad 0.10$ & $\mathrm{U}$ & $0.10 \mathrm{~L}$ & & $0.10 \mathrm{U}$ \\
\hline Dibenzothiophene & 0.40 & 1.82 & $1.79 \mathrm{~J}$ & $0.40 \mathrm{U}$ & $0.40 \mathrm{~L}$ & & $0.40 \mathrm{U}$ \\
\hline C1-Dibenzothiophenes & 0.40 & 1.82 & $8.49 \quad 0.40$ & $\mathrm{U}$ & $0.40 \mathrm{~L}$ & & $0.98 \mathrm{~J}$ \\
\hline C2-Dibenzothiophenes & 0.40 & 1.82 & $25.9 \quad 0.40$ & $\mathrm{U}$ & 7.57 & 6.09 & \\
\hline C3-Dibenzothiophenes & 0.40 & 1.82 & 22.72 .53 & 7.78 & & 8.28 & \\
\hline C4-Dibenzothiophenes & 0.40 & 1.82 & $14.8 \quad 0.40$ & $\mathrm{U}$ & $0.40 \mathrm{~L}$ & & 10.8 \\
\hline Fluoranthene & 0.81 & 1.82 & $84.0 \quad 6.47$ & 10.8 & & 12.3 & \\
\hline Pyrene & 0.35 & 1.82 & 47.94 .06 & 6.48 & & 8.72 & \\
\hline C1-Fluoranthenes/Pyrenes & 0.81 & 1.82 & 33.74 .98 & 10.9 & & 9.43 & \\
\hline C2-Fluoranthenes/Pyrenes & 0.81 & 1.82 & $16.2 \quad 2.44$ & 0.81 & & $\mathrm{U}$ & 5.68 \\
\hline C3-Fluoranthenes/Pyrenes & 0.81 & 1.82 & $0.81 \mathrm{U}$ & $0.81 \mathrm{U}$ & $0.81 \mathrm{~L}$ & & $0.81 \mathrm{U}$ \\
\hline Benzo(a)anthracene & 0.54 & 1.82 & 22.72 .68 & 4.52 & & 6.00 & \\
\hline Chrysene & 0.87 & 1.82 & $42.2 \quad 4.50$ & 5.64 & & 9.84 & \\
\hline C1-Chrysenes & 0.87 & 1.82 & 11.93 .67 & 7.21 & & 6.98 & \\
\hline C2-Chrysenes & 0.87 & 1.82 & $7.68 \quad 3.93$ & 5.02 & & 8.27 & \\
\hline C3-Chrysenes & 0.87 & 1.82 & $0.87 \mathrm{U}$ & $0.87 \mathrm{U}$ & $0.87 \mathrm{~L}$ & & 3.42 \\
\hline C4-Chrysenes & 0.87 & 1.82 & $0.87 \mathrm{U}$ & $0.87 \mathrm{U}$ & $0.87 \mathrm{~L}$ & $\mathrm{U}$ & $0.87 \mathrm{U}$ \\
\hline Benzo(b)fluoranthene & 0.37 & 1.82 & $17.4 \quad 3.25$ & 3.01 & & 6.02 & \\
\hline Benzo(k)fluoranthene & 0.21 & 1.82 & 6.091 .19 & $\mathrm{~J}$ & $1.20 \mathrm{~J}$ & & 2.15 \\
\hline Benzo(e)pyrene & 0.22 & 1.82 & 12.51 .92 & 2.46 & & 4.58 & \\
\hline Benzo(a)pyrene & 0.23 & 1.82 & 3.291 .09 & $\mathrm{~J}$ & $0.99 \mathrm{~J}$ & & $1.81 \mathrm{~J}$ \\
\hline Perylene & 0.25 & 1.82 & $2.24 \quad 0.71$ & $\mathrm{~J}$ & $0.82 \mathrm{~J}$ & & $1.13 \mathrm{~J}$ \\
\hline Indeno(1,2,3-cd)pyrene & 0.14 & 1.82 & $2.01 \quad 0.91$ & $\mathrm{~J}$ & $0.44 \mathrm{~J}$ & & $1.10 \mathrm{~J}$ \\
\hline Dibenz(a,h)anthracene & 0.06 & 1.82 & $0.06 \mathrm{U}$ & $0.06 \mathrm{U}$ & $0.06 \mathrm{~L}$ & & $0.06 \mathrm{U}$ \\
\hline Benzo(g,h,i)perylene & 0.22 & 1.82 & $2.32 \quad 1.18$ & $\mathrm{~J}$ & $0.64 \mathrm{~J}$ & & $1.66 \mathrm{~J}$ \\
\hline
\end{tabular}


BATTELLE MARINE SCIENCE LABORATORIES

1529 West Sequim Bay Road

Sequim, Washington 98382-9099

360/681-4564
2010 Regional Mussel Watch - AMB02

ENVVEST 2010

PAHs in Indigenous Mussels

UNITS: ng/g wet wt.

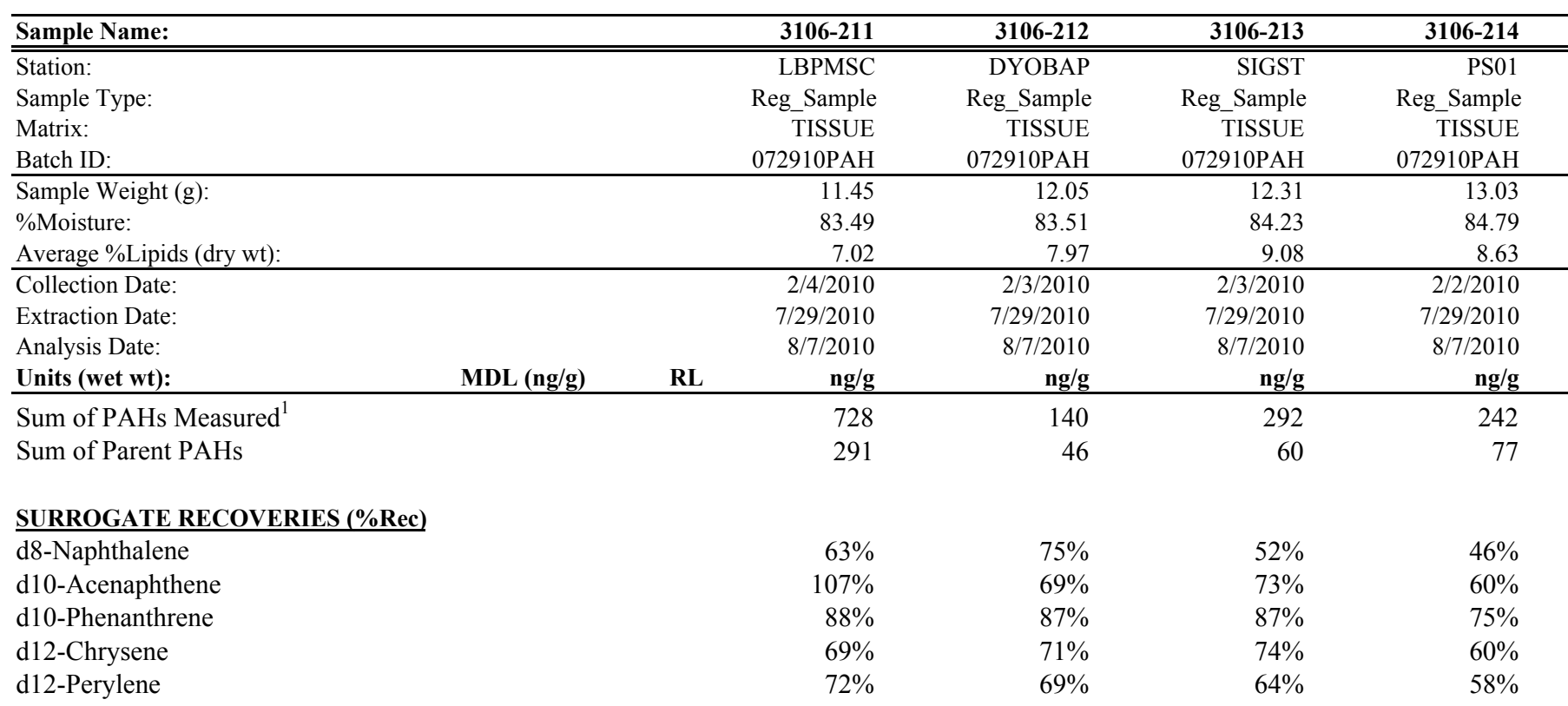

${ }^{1}$ Sum of PAHs includes the MDL for non-detects 
BATTELLE MARINE SCIENCE LABORATORIES

1529 West Sequim Bay Road

Sequim, Washington 98382-9099

$360 / 681-4564$

\section{ENVVEST \\ Biota Studies \\ 2010 Mussel Study \\ Stable Isotopes}

\begin{tabular}{|c|c|c|c|c|c|c|c|}
\hline Station Location & Description & \begin{tabular}{|l} 
Station \\
Code
\end{tabular} & Organism & MSL Code & \begin{tabular}{|c|c|}
$\begin{array}{c}\text { Collection } \\
\text { Date }\end{array}$ \\
\end{tabular} & $\delta^{15} \mathbf{N}$ & $\delta^{13} \mathrm{C}$ \\
\hline PSNS & PNSN Inactive Fleet Callow Ave OF & PS01 & Mussel & $3106-238$ & $02 / 02 / 10$ & 11.01 & -20.57 \\
\hline PSNS & PSNS NAVSTA Mid (CP) & PS04 & Mussel & $3106-215$ & $01 / 12 / 10$ & 9.74 & -18.65 \\
\hline PSNS & PSNS NAVSTA West (DP) & PS03 & Mussel & $3106-216$ & $01 / 12 / 10$ & 9.93 & -18.68 \\
\hline PSNS & PSNS CIA West (6) & PS06 & Mussel & $3106-217$ & $01 / 12 / 10$ & 10.05 & -18.69 \\
\hline PSNS & PSNS CIA MidE (5) & PS08 & Mussel & $3106-218$ & $01 / 12 / 10$ & 10.03 & -18.72 \\
\hline PSNS & PSNS CIA MidW (4) & PS09 & Mussel & $3106-219$ & $01 / 12 / 10$ & 10.01 & -17.48 \\
\hline PSNS & PSNS CIA East (3) & PS11 & Mussel & $3106-220$ & $01 / 12 / 10$ & 9.90 & -18.17 \\
\hline Sinclair Inlet & Sinclair Inlet Sinclair Marina & SISIM & Mussel & $3106-221$ & $01 / 12 / 10$ & 9.81 & -19.02 \\
\hline Sinclair Inlet & Sinclair Inlet Port Orchard Marina & SIPOM & Mussel & $3106-223$ & $01 / 12 / 10$ & 10.15 & -18.05 \\
\hline Sinclair Inlet & Sinclair Inlet Waterman Point & SIWP & Mussel & $3106-228$ & $01 / 08 / 10$ & 9.93 & -17.74 \\
\hline Sinclair Inlet & Sinclair Inlet Ross Point & SIRP & Mussel & $3106-229$ & $01 / 08 / 10$ & 10.12 & -18.42 \\
\hline Sinclair Inlet & Sinclair Inlet head at Gorst & SIGST & Mussel & $3106-237$ & $02 / 03 / 10$ & 10.44 & -22.41 \\
\hline Port Orchard & Port Orchard Pass Illahee State Park & POPISP & Mussel & $3106-222$ & $01 / 12 / 10$ & 9.44 & -18.48 \\
\hline Port Orchard & Port Orchard Pass Illahee State Dock & POPIPD & Mussel & $3106-224$ & $01 / 12 / 10$ & 9.42 & -18.17 \\
\hline Port Orchard & Port Orchard Passage Brownsville & POPBWN & Mussel & $3106-230$ & $02 / 04 / 10$ & 10.69 & -20.02 \\
\hline Dyes Inlet & Port Washington Narrows Lions Park & PWNLP & Mussel & $3106-225$ & $01 / 12 / 10$ & 10.10 & -17.84 \\
\hline Dyes Inlet & Dyes Inlet Old Town Silverdale & DYOTS & Mussel & $3106-226$ & $01 / 12 / 10$ & 10.12 & -18.63 \\
\hline Dyes Inlet & Dyes Inlet Ostrich Bay Ammo Pier & DYOBAP & Mussel & $3106-236$ & $02 / 03 / 10$ & 10.72 & -18.90 \\
\hline Rich Passage & Manchester Lab Pier & MLPIER & Mussel & $3106-227$ & $01 / 08 / 10$ & 10.39 & -18.27 \\
\hline Keyport Passage & Keyport NUWC Pier & KPTPIER & Mussel & $3106-231$ & $02 / 04 / 10$ & 9.99 & -18.96 \\
\hline Keyport Passage & Keyport Lagoon & KPTLAG & Mussel & $3106-232$ & $02 / 04 / 10$ & 10.10 & -20.26 \\
\hline Keyport Passage & Liberty Bay Poulsbo Marina Science Center & LBPMSC & Mussel & $3106-235$ & $02 / 04 / 10$ & 9.36 & -21.35 \\
\hline Agate Passage & Agate Pass BI Hidden Cove Beach & APHCB & Mussel & $3106-233$ & $02 / 04 / 10$ & 10.66 & -18.92 \\
\hline Agate Passage & Agate Pass Kiana Lodge & APKIANA & Mussel & $3106-234$ & $02 / 04 / 10$ & 11.44 & -18.97 \\
\hline
\end{tabular}




\section{QA/QC Sample Results}


ENVVEST 2010

\begin{tabular}{|c|c|c|c|c|c|c|c|c|}
\hline Sample ID - Metals & Station Code & Fraction & $\begin{array}{c}\text { Sample } \\
\text { Type }\end{array}$ & MSL Code & $\begin{array}{c}\text { Collection } \\
\text { Date }\end{array}$ & $\begin{array}{c}\text { \# Mussels } \\
\text { in Comp }\end{array}$ & $\begin{array}{c}\text { Average } \\
\text { lenth } \\
(\mathrm{mm})\end{array}$ & $\begin{array}{l}\text { Percent } \\
\text { Moistur }\end{array}$ \\
\hline
\end{tabular}

Metals in Tissue

UITS: $\mu \mathrm{g} / \mathrm{g}$ dry wt.

Laboratory Achieved Method Detection Limits (tissue)

Reporting Limit (MDL* 3.18)

Instrument:

\section{METHOD BLANKS}

MB-1

MB-2

\section{LABORATORY CONTROL SAMPLES}

MB-1

LCS-1

MB-2

LCS-1

Blank R1 041410

Blank R2 041410

Blank R1 041410

LCS R 1041410

Spiking Level

Percent Recovery, LCS

Blank R2 041410
LCS R2 041410
Spiking Level

Percent Recovery, LCS

\section{MATRIX SPIKE RESULTS}

20100112MUS05-C PS09

MS-1

MSD-1

$3106-21$ 3106-219MS

3106-219MSD

Spiking Level, MS

Spiking Level, MSD

Percent Recovery, MS

Percent Recovery, MSD

RPD

MFEB4-029C

MS-2

MSD-2

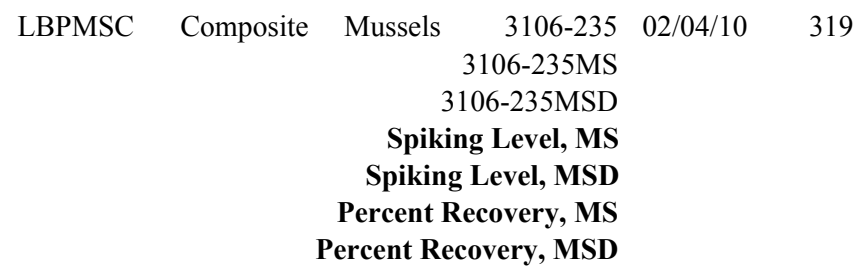

RPD

\begin{tabular}{rrrrrr} 
Ag & As & Cd & Pb & Cr & Cu \\
\hline$I C P-M S$ & $I C P-M S$ & $I C P-M S$ & $I C P-M S$ & $I C P-O E S$ & $I C P-O E S$ \\
\hline $\mathbf{0 . 0 0 3 1}$ & $\mathbf{0 . 1 1}$ & $\mathbf{0 . 0 0 2 9}$ & $\mathbf{0 . 0 0 3 4}$ & $\mathbf{0 . 0 3 5}$ & $\mathbf{0 . 0 9 4}$ \\
$\mathbf{0 . 0 1 0}$ & $\mathbf{0 . 3 5}$ & $\mathbf{0 . 0 0 9 2}$ & $\mathbf{0 . 0 1 1}$ & $\mathbf{0 . 1 1}$ & $\mathbf{0 . 3 0}$ \\
\hline
\end{tabular}

$\begin{array}{rccccc}0.0031 \mathrm{U} & 0.126 \mathrm{~J} & 0.0029 \mathrm{U} & 0.0034 \mathrm{U} & 0.035 \mathrm{U} & 0.094 \mathrm{U} \\ 0.0031 \mathrm{U} & 0.250 \mathrm{~J} & 0.0029 \mathrm{U} & 0.0034 \mathrm{U} & 0.035 \mathrm{U} & 0.094 \mathrm{U} \\ & & & & & \\ & & & & & \\ 0.0031 \mathrm{U} & 0.126 \mathrm{~J} & 0.0029 \mathrm{U} & 0.0034 \mathrm{U} & 0.035 \mathrm{U} & 0.094 \mathrm{U} \\ 2.00 & 1.93 & 1.96 & 2.04 & 2.03 & 1.98 \\ 2 & 2 & 2 & 2 & 2 & 2 \\ \mathbf{1 0 0 \%} & \mathbf{9 0 \%} & \mathbf{9 8 \%} & \mathbf{1 0 2 \%} & \mathbf{1 0 2 \%} & \mathbf{9 9 \%} \\ & & & & & \\ 0.0031 \mathrm{U} & 0.250 \mathrm{~J} & 0.0029 \mathrm{U} & 0.0034 \mathrm{U} & 0.035 \mathrm{U} & 0.094 \mathrm{U} \\ 1.89 & 2.17 & 1.94 & 1.92 & 2.04 & 1.96 \\ 2 & 2 & 2 & 2 & 2 & 2 \\ \mathbf{9 4 \%} & \mathbf{9 6 \%} & \mathbf{9 7 \%} & \mathbf{9 6 \%} & \mathbf{1 0 2 \%} & \mathbf{9 8 \%}\end{array}$

\begin{tabular}{|c|c|c|c|c|c|c|c|c|c|}
\hline \multirow{8}{*}{$01 / 12 / 10$} & 123 & 48.7 & 88.7 & 0.0189 & 6.90 & 2.12 & 1.60 & 0.949 & 13.3 \\
\hline & & & & 1.93 & 33.4 & 4.31 & 3.67 & 3.00 & 40.6 \\
\hline & & & & 1.92 & 33.6 & 4.21 & 3.72 & 2.93 & 40.8 \\
\hline & & & & 2.11 & 26.3 & 2.11 & 2.11 & 2.11 & 26.3 \\
\hline & & & & 2.13 & 26.7 & 2.13 & 2.13 & 2.13 & 26.7 \\
\hline & & & & $91 \%$ & $101 \%$ & $104 \%$ & $99 \%$ & $97 \%$ & $104 \%$ \\
\hline & & & & $89 \%$ & $100 \%$ & $98 \%$ & $99 \%$ & $93 \%$ & $103 \%$ \\
\hline & & & & $2.0 \%$ & $0.8 \%$ & $5.7 \%$ & $0.8 \%$ & $4.9 \%$ & $0.6 \%$ \\
\hline \multirow[t]{8}{*}{$02 / 04 / 10$} & 319 & 33.0 & 83.5 & 0.0193 & 9.51 & 2.65 & 1.20 & 1.24 & 23.7 \\
\hline & & & & 1.87 & 34.5 & 27.0 & 3.29 & 3.25 & 48.8 \\
\hline & & & & 1.83 & 33.7 & 27.1 & 3.14 & 3.14 & 48.8 \\
\hline & & & & 2.06 & 25.2 & 25.2 & 2.06 & 2.06 & 25.2 \\
\hline & & & & 2.00 & 25.5 & 25.5 & 2.00 & 2.00 & 25.5 \\
\hline & & & & $90 \%$ & $99 \%$ & $97 \%$ & $102 \%$ & $98 \%$ & $99 \%$ \\
\hline & & & & $91 \%$ & $95 \%$ & $96 \%$ & $97 \%$ & $95 \%$ & $98 \%$ \\
\hline & & & & $1.0 \%$ & $4.3 \%$ & $0.8 \%$ & $4.4 \%$ & $2.5 \%$ & $1.0 \%$ \\
\hline
\end{tabular}


BATTELLE MARINE SCIENCE LABORATORIES

1529 West Sequim Bay Road

2010 Regional Mussel Watch - AMB02

Sequim, Washington 98382-9099

ENVVEST 2010

PM: Jill Brandenberger 360/681-4564

Metals in Tissue

UNITS: $\mu \mathrm{g} / \mathrm{g}$ dry wt.

\begin{tabular}{|c|c|c|c|c|c|c|c|c|c|c|}
\hline Sample ID - Metals & Station Code & Fraction & $\begin{array}{l}\text { Sample } \\
\text { Type }\end{array}$ & MSL Code & $\mathbf{N i}$ & Zn & $\mathbf{H g}$ & $\begin{array}{c}\text { CVAA } \\
\text { Batch ID }\end{array}$ & $\begin{array}{l}\text { ICP-OES } \\
\text { Batch ID }\end{array}$ & $\begin{array}{l}\text { ICP-MS } \\
\text { Batch ID }\end{array}$ \\
\hline & & & & Instrument: & ICP-OES & ICP-OES & $\overline{C V A A}$ & & & \\
\hline \multirow{2}{*}{\multicolumn{4}{|c|}{$\begin{array}{l}\text { Laboratory Achieved Method Detection Limits (tissue) } \\
\text { Reporting Limit (MDL*3.18) }\end{array}$}} & & 0.041 & 0.018 & 0.0044 & & & \\
\hline & & & & & 0.13 & 0.057 & 0.014 & & & \\
\hline
\end{tabular}

\section{METHOD BLANKS

MB-1

MB-2

\section{LABORATORY CONTROL SAMPLES \\ MB-1}

LCS-1

MB-2

LCS-1

\section{MATRIX SPIKE RESULTS \\ 20100112MUS05-C PS09}

MS-1

MSD-1

Composite

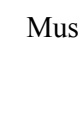

Blank R1 041410

Blank R2 041410

$0.041 \mathrm{U}$

$0.018 \mathrm{U}$

$0.00950 \mathrm{~J}$

050610HGBL

050610HGBL

I042310A

I042310A

042110-6100

042110-6100

Blank R1 041410

$0.041 \mathrm{U}$

$0.018 \mathrm{U}$

LCS R1 041410

2.0

Spiking Level

2

Percent Recovery, LCS

Blank R2 041410

LCS R2 041410

Spiking Level

$0.041 \mathrm{U}$

2.07

$103 \%$

Percent Recovery, LCS

$$
2.00
$$

2

$0.00950 \mathrm{~J}$

1.99

050610HGBL

I042310A

I042310A

042110-6100

1.99
2

050610HGBL

$0.025 \mathrm{~J}$

2.04

0.00820

1.94

050610HGBL

050610HGBL

I042310A

I042310A

042110-6100

$101 \%$

97\%

(n)

MFEB4-029C

MS-2

MSD-2
050610HGBL 050610HGBL 050610HGBL

I042310A

I042310A

I042310A

042110-6100

042110-6100

042110-6100

050610HGBL

050610HGBL

I042310A

I042310A

I042310A

042110-6100

042110-6100

042110-6100

\section{Mussels 3106-219} 3106-219MS 3106-219MSD

Spiking Level, MS

Spiking Level, MSD

Percent Recovery, MS

Percent Recovery, MSD

RPD

0.857

2.86

2.98

2.11

2.13

$95 \%$

$100 \%$

$4.5 \%$

Mussels 3106-235 3106-235MS 3106-235MSD

Spiking Level, MS

Spiking Level, MSD

Percent Recovery, MS

Percent Recovery, MSD

RPD

1.26
3.21
2.93
2.06
2.00
$\mathbf{9 5 \%}$
$\mathbf{8 4 \%}$

1.26

3.21

2.93
2.06

2.00

$84 \%$

$12.5 \%$

210
355
364
132
133
$\mathbf{1 1 0 \%}$
$\mathbf{1 1 6 \%}$
$\mathbf{5 . 3 \%}$

$5.3 \%$

239

369

358

126

127

$103 \%$

$94 \%$

$9.6 \%$

$$
\begin{array}{r}
0.140 \\
2.16 \\
2.13 \\
2.11 \\
2.13 \\
\mathbf{9 6 \%} \\
\mathbf{9 3 \%} \\
\mathbf{2 . 7 \%}
\end{array}
$$

0.140

2.10

1.99

2.06

2.00

$\mathbf{9 5 \%}$

$93 \%$

$2.7 \%$ 
2010 Regional Mussel Watch - AMB02

PM: Jill Brandenberger 360/681-4564

ENVVEST 2010

Metals in Tissue

\begin{tabular}{|c|c|c|c|c|c|c|c|c|c|c|c|c|c|c|}
\hline Sample ID - Metals & Station Code & Fraction & $\begin{array}{c}\text { Sample } \\
\text { Type }\end{array}$ & MSL Code & $\begin{array}{c}\text { Collection } \\
\text { Date }\end{array}$ & $\begin{array}{c}\text { \# Mussels } \\
\text { in Comp }\end{array}$ & $\begin{array}{c}\text { Average } \\
\text { lenth } \\
(\mathrm{mm})\end{array}$ & $\begin{array}{l}\text { Percent } \\
\text { Moisture }\end{array}$ & Ag & As & Cd & $\mathbf{P b}$ & $\mathrm{Cr}$ & $\mathrm{Cu}$ \\
\hline & & & & Instrument: & & & & & ICP-MS & $I C P-M S$ & $I C P-M S$ & $I C P-M S$ & ICP-OES & ICP-OES \\
\hline \multicolumn{15}{|c|}{ REPLICATE PRECISION } \\
\hline 20100112MUS01-C & PS04 & Composite & Mussels & $3106-215$ & $01 / 12 / 10$ & 194 & 45.3 & 86.6 & 0.00945 & 6.26 & 1.82 & 1.25 & 0.766 & 10.3 \\
\hline \multirow[t]{3}{*}{ 20100112MUS01-C } & PS04 & Composite & Mussels & $3106-215 \mathrm{r} 2$ & $01 / 12 / 10$ & 194 & 45.3 & 86.6 & 0.00967 & 6.08 & 1.83 & 1.21 & 0.777 & 10.2 \\
\hline & & & & Mean & & & & & 0.00956 & 6.17 & 1.83 & 1.23 & 0.772 & 10.3 \\
\hline & & & & RPD & & & & & $2.3 \%$ & $3.0 \%$ & $0.4 \%$ & $3.3 \%$ & $1.4 \%$ & $0.5 \%$ \\
\hline AMB02-102C & PS01 & Composite & Mussels & $3106-238$ & $02 / 02 / 10$ & 272 & 42.0 & 84.8 & 0.0236 & 6.73 & 2.69 & 1.26 & 1.09 & 11.6 \\
\hline \multirow[t]{3}{*}{ AMB02-102C } & PS01 & Composite & Mussels & $3106-238 \mathrm{r} 2$ & $02 / 02 / 10$ & 272 & 42.0 & 84.8 & 0.0249 & 6.68 & 2.72 & 1.28 & 1.11 & 11.7 \\
\hline & & & & Mean & & & & & 0.0243 & 6.70 & 2.71 & 1.27 & 1.10 & 11.7 \\
\hline & & & & RPD & & & & & $5.6 \%$ & $0.8 \%$ & $1.3 \%$ & $1.7 \%$ & $1.9 \%$ & $1.6 \%$ \\
\hline \multicolumn{15}{|c|}{ STANDARD REFERENCE MATERIAL } \\
\hline & & & 156 & fb R1 041410 & & & & 0.0 & 0.599 & 6.61 & 2.35 & 0.307 & 0.480 & 68.5 \\
\hline \multirow[t]{4}{*}{ SRM-2 } & & & 156 & fb R2 041410 & & & & 0.0 & 0.576 & 6.91 & 2.38 & 0.298 & 0.457 & 67.8 \\
\hline & & & & rtified Value & & & & & 0.666 & 7.65 & 2.48 & 0.308 & $\mathrm{NC}$ & 71.6 \\
\hline & & & Perce & at Difference & & & & & $10 \%$ & $14 \%$ & $5 \%$ & $0 \%$ & & $4 \%$ \\
\hline & & & Perce & ht Difference & & & & & $14 \%$ & $10 \%$ & $4 \%$ & $3 \%$ & & $5 \%$ \\
\hline
\end{tabular}


BATTELLE MARINE SCIENCE LABORATORIES

1529 West Sequim Bay Road

2010 Regional Mussel Watch - AMB02

Sequim, Washington 98382-9099

ENVVEST 2010

PM: Jill Brandenberger 360/681-4564

Metals in Tissue

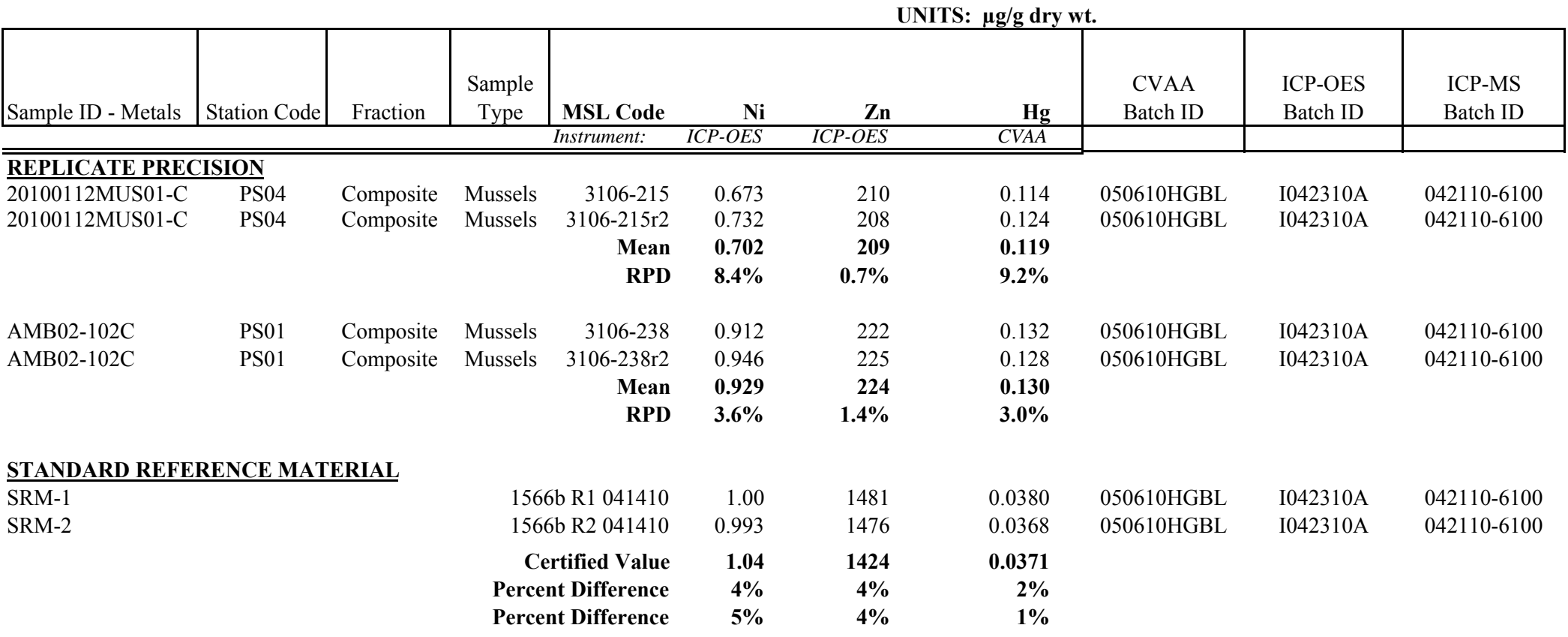


BATTELLE MARINE SCIENCE LABORATORIES

1529 West Sequim Bay Road

Sequim, Washington 98382-9099

ENVVEST 2010_AMB02

$360 / 681-4564$

Metals in Mussel Samples

DATA QUALIFIERS:

c Exceeds DQO but meets contingency criteria of either:

1 SRM certified $<10 x$ MDL

2 Insufficient spiking level relative to native sample concentrations

3 Sample concentration $<10 x \mathrm{MDL}$

$\mathrm{U}$ Analyte not detected at or above the MDL, MDL reported

J Analyte detected above the MDL, but less than the RL

-- Not analyzed

NA Not applicable/available

N Spiked sample recovery outside QC criterion of 70-130\%

NC Not Certified

\& Accuracy result outside QC criterion of $\leq 20 \%$ PD

* Precision result outside QC criterion of $<30 \%$

NS Sample not spiked for this analyte

B Analyte detected in the method blank $>$ RL and sample concentration $<10$ times detected blank value

b Data are blank corrected using the batch specific procedural blank 
BATTELLE MARINE SCIENCE LABORATORIES

1529 West Sequim Bay Road

Sequim, Washington 98382-9099

360/681-4564

Sample Name:

Sample Type;

\begin{tabular}{l} 
Matrix: \\
Batch ID: \\
Sample W \\
Extraction \\
Analysis D \\
Units (wet \\
\hline \\
C12(8) \\
C13(18) \\
C13(28) \\
C14(44) \\
C14(52) \\
C14(66) \\
C14(77) \\
C15(101) \\
C15(105) \\
C15(118) \\
C15(126) \\
C16(128) \\
C16(138) \\
C16(153) \\
C17(170) \\
C17(180) \\
C17(187) \\
C18(195) \\
C18(200) \\
C19(206) \\
C110(209)
\end{tabular}

C110(209)

BLK070210
$\mathrm{MB}$
Diatomaceous
earth \& Sodium
Sulfate $^{1}$
$070210 \mathrm{PCB}$

2010 Regional Mussel Watch - AMB02 ENVVEST 2010

PCBs in Indigenous Mussels

UNITS: ng/g wet wt.

QC Summary

\section{SURROGATE}

\section{RECOVERY}

(\%Rec)

$\mathrm{Cl} 3(30)$

Cl4(65)

Cl8(198)

BLK070610
MB

Diatomaceous

earth \& Sodium

Sulfate $^{1}$

070610PCB

devided by $10 \mathrm{~g}$

$7 / 2 / 2010$

$7 / 8 / 2010$

$\mathrm{ng} / \mathrm{g}$

$0.16 \mathrm{U}$

$0.15 \mathrm{U}$

$0.02 \mathrm{U}$

$0.02 \mathrm{U}$

$0.05 \mathrm{U}$

$0.08 \mathrm{U}$

$0.15 \mathrm{U}$

$0.17 \mathrm{U}$

$0.03 \mathrm{U}$

$0.10 \mathrm{U}$

$0.07 \mathrm{U}$

$0.04 \mathrm{U}$

$0.13 \mathrm{U}$

$0.18 \mathrm{U}$

$0.02 \mathrm{U}$

$0.03 \mathrm{U}$

$0.08 \mathrm{U}$

$0.01 \mathrm{U}$

$0.08 \mathrm{U}$

$0.01 \mathrm{U}$

$0.01 \mathrm{U}$ devided by $10 \mathrm{~g}$

$7 / 6 / 2010$

$8 / 3 / 2010$
BLK071910
MB

Diatomaceous

earth \& Sodium

Sulfate $^{1}$

071910PCB

devided by $10 \mathrm{~g}$

$7 / 19 / 2010$

$8 / 4 / 2010$

$$
\begin{array}{r}
\text { BLK072910 } \\
\text { MB } \\
\text { Diatomaceous } \\
\text { earth \& Sodium } \\
\text { Sulfate }^{1} \\
\text { 072910PCB }
\end{array}
$$

devided by $10 \mathrm{~g}$

$7 / 29 / 2010$

$8 / 6 / 2010$

$\mathrm{ng} / \mathrm{g}$

$0.16 \mathrm{U}$

$0.15 \mathrm{U}$

$0.02 \mathrm{U}$

$0.02 \mathrm{U}$

$0.05 \mathrm{U}$

$0.08 \mathrm{U}$

$0.15 \mathrm{U}$

$0.17 \mathrm{U}$

$0.03 \mathrm{U}$

$0.10 \mathrm{U}$

$0.07 \mathrm{U}$

$0.04 \mathrm{U}$

$0.13 \mathrm{U}$

$0.18 \mathrm{U}$

$0.02 \mathrm{U}$

$0.03 \mathrm{U}$

$0.08 \mathrm{U}$

$0.01 \mathrm{U}$

$0.08 \mathrm{U}$

$0.01 \mathrm{U}$

$0.01 \mathrm{U}$
BLK080610

MB

Diatomaceous

earth \& Sodium

Sulfate $^{1}$

080610PCB

devided by $10 \mathrm{~g}$

$8 / 6 / 2010$

$8 / 10 / 2010$

$\mathrm{ng} / \mathrm{g}$

$0.16 \mathrm{U}$

$0.15 \mathrm{U}$

$0.02 \mathrm{U}$

$0.02 \mathrm{U}$

$0.05 \mathrm{U}$

$0.08 \mathrm{U}$

$0.15 \mathrm{U}$

$0.17 \mathrm{U}$

$0.03 \mathrm{U}$

$0.10 \mathrm{U}$

$0.07 \mathrm{U}$

$0.04 \mathrm{U}$

$0.13 \mathrm{U}$

$0.18 \mathrm{U}$

$0.02 \mathrm{U}$

$0.03 \mathrm{U}$

$0.08 \mathrm{U}$

$0.01 \mathrm{U}$

$0.08 \mathrm{U}$

$0.01 \mathrm{U}$

$0.01 \mathrm{U}$

\footnotetext{
${ }^{1}$ Diatomaceous earth \& Sodium Sulfate are the drying and dispersing agents (mixed with sample) used in the extraction
} 
BATTELLE MARINE !

1529 West Sequim Ba

Sequim, Washington !

360/681-4564

Sample Name:

Sample Type;

Matrix:

Batch ID:

TISSUE

070210PCB

Sample Weight (g):

11.99

Extraction Date:

Analysis Date:

Units (wet wt):

C12(8)

$\mathrm{Cl3}(18)$

$\mathrm{Cl3}$ (28)

C14(44)

C14(52)

C14(66)

C14(77)

C15(101)

C15(105)

$\mathrm{Cl5}(118)$

$\mathrm{Cl5}(126)$

Cl6(128)

Cl6(138)

Cl6(153)

$\mathrm{Cl7}(170)$

$\mathrm{C17}(180)$

$\mathrm{Cl} 7(187)$

$\mathrm{Cl}$ (195)

$\mathrm{Cl}$ (200)

C19(206)

Cl10(209)

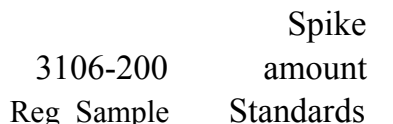

$3106-$
200 SPK1
MS

2010 Regional Mussel Watch - AMB02

ENVVEST 2010

PCBs in Indigenous Mussels

UNITS: ng/g wet wt.

QC Summary

$\begin{array}{rrrr}3106- & \text { Spike } & 3106- & 3106- \\ \text { 200SPK1 } & \text { amount } & \text { 200SPK2 } & \text { 200SPK2 } \\ \text { MS } & \text { Standards } & \text { MS } & \text { MS }\end{array}$

\section{SURROGATE}

\section{RECOVERY}

(\%Rec)

$\mathrm{Cl3}(30)$

C14(65)

$\mathrm{Cl}$ (198)

$\begin{array}{ll}89 \% & 75 \% \\ 96 \% & 85 \% \\ 92 \% & 79 \%\end{array}$

$82 \%$

$92 \%$

$82 \%$

${ }^{1}$ Diatomaceous earth c 
BATTELLE MARINE :

1529 West Sequim Ba

Sequim, Washington !

360/681-4564
2010 Regional Mussel Watch - AMB02

ENVVEST 2010

PCBs in Indigenous Mussels

UNITS: $n g / g$ wet wt.

QC Summary

$\begin{array}{rr}\text { SIPOM 3106- } & \text { SIPOM 3106- } \\ 199 & 199 B \\ \text { Reg_Sample } & \text { Duplicate }\end{array}$

$\begin{array}{rr}\text { SIWP 3106- } & \text { SIWP 3106- } \\ 204 & 204 \mathrm{~B} \\ \text { Reg_Sample } & \text { Duplicate }\end{array}$

Sample Name:

Reg_Sample

Matrix:

TISSUE

070210PCB

TISSUE

070210PCB

Sample Weight (g):

11.45

11.44

$7 / 2 / 2010$

$7 / 2 / 2010$

$7 / 8 / 2010$

$7 / 8 / 2010$ $\mathrm{ng} / \mathrm{g}$

0.24

0.20

0.21

0.27

0.61

0.46

0.85

1.91

0.57

1.75

0.84

0.38

2.24

2.53

$0.10 \mathrm{~J}$

$0.363 \%$

$0.920 \%$

$0.01 \mathrm{U}$

$0.37 \quad 4 \%$

$0.01 \mathrm{U}$

$0.01 \mathrm{U}$
TISSUE

071910PCB

11.59

$7 / 19 / 2010$

$8 / 4 / 2010$

$\mathrm{ng} / \mathrm{g}$

$0.17 \mathrm{~J}$

$0.15 \mathrm{U}$

$0.08 \mathrm{~J}$

$0.10 \mathrm{~J}$

$\begin{array}{ll}0.25 & 0.23\end{array}$

$0.14 \mathrm{~J}$

$\begin{array}{ll}0.39 & 0.36\end{array}$

0.820 .85

$0.20 \mathrm{~J}$

$\begin{array}{ll}0.78 & 0.75\end{array}$

$\begin{array}{ll}0.68 & 0.65\end{array}$

$\begin{array}{ll}0.24 & 0.22\end{array}$

$\begin{array}{ll}1.33 & 1.32\end{array}$

$1.70 \quad 1.64$

$0.11 \mathrm{~J}$

0.26

0.71

$0.01 \mathrm{U}$

0.24

$0.01 \mathrm{U}$

$0.01 \mathrm{U}$
TISSUE

072910PCB

11.12

$7 / 29 / 2010$

$8 / 6 / 2010$

$\mathrm{ng} / \mathrm{g}$

$0.18 \mathrm{~J}$

$0.15 \mathrm{U}$

$0.07 \mathrm{~J}$

$0.10 \mathrm{~J}$

$0.14 \mathrm{~J}$

.

$0.19 \mathrm{~J}$

.

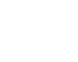

$0.09 \mathrm{~J}$

0.26

0.71

$0.01 \mathrm{U}$

0.24

$0.01 \mathrm{U}$

$0.01 \mathrm{U}$ $\mathrm{ng} / \mathrm{g}$

0.25

0.21

$0.20 \mathrm{~J}$

0.27

0.62

C14(52)

C14(66)

C14(77)

C15(101)

$\mathrm{Cl5}(105)$

$\mathrm{Cl5}(118)$

Cl5(126)

Cl6(128)

Cl6(138)

Cl6(153)

Cl7(170)

$\mathrm{Cl}$ (180)

C17(187)

$\mathrm{Cl}$ (195)

$\mathrm{Cl}$ (200)

C19(206)

C110(209)
0.47

0.87

1.83

0.56

1.79

0.86

0.41

2.21

2.55

$0.09 \mathrm{~J}$

0.35

0.92

$0.01 \mathrm{U}$

0.35

$0.01 \mathrm{U}$

$0.01 \mathrm{U}$
RPD

\section{SURROGATE}

\section{RECOVERY}

(\%Rec)

$\mathrm{Cl} 3(30)$

Cl4(65)

Cl8(198)

$\begin{array}{ll}87 \% & 86 \% \\ 99 \% & 94 \% \\ 75 \% & 71 \%\end{array}$

$\begin{array}{ll}78 \% & 87 \% \\ 92 \% & 99 \% \\ 70 \% & 76 \%\end{array}$

${ }^{1}$ Diatomaceous earth $c$ 
BATTELLE MARINE SCIENCE LABORATORIES

1529 West Sequim Bay Road

Sequim, Washington 98382-9099

2010 Regional Mussel Watch - AMB02

ENVVEST 2010

$360 / 681-4564$

PCBs in Indigenous Mussels

Data Qualifiers for PCBs

\section{Data Qualifiers}

$\mathrm{U} \quad$ Not detected at or above laboratory achieved detection limit; MDL reported

$\mathrm{J} \quad$ Analyte concentration is less that the RL, but greater than the MDL

B Analyte detected in the method blank above the RL, sample concentration $<10 \mathrm{x}$ detected blank value

\& Outside Project DQO for spike recovery (40-120\%), replicate analysis $(<30 \%)$, or SRM PD $(<30 \%)$

D Results determined from dilution

c Exceeds Project DQO but meets contingency criteria

\# Outside Project DQOs for surrogate Recovery (40-120\%)

* $\quad$ Associated surrogate recovery exceeds DQO guidelines

${ }^{1}$ NOAA Status and Trends method of summing congeners (sum PCB congeners*2) [NOAA, 1995; O’Connor, 2002].

NOAA (1995). Magnitude and Extent of Sediment Toxicity in the Hudson-Raritan Estuary. NOAA Tech. Memo. NOS ORCA 88. Silver Springs, MD. 242pp.

O'Connor, T.P. (2002). National distribution of chemical concentrations in mussels and oysters in the USA. Marine Environmental Research 53:117-143. 
BATTELLE MARINE SCIENCE LABORATORIES

1529 West Sequim Bay Road

Sequim, Washington 98382-9099

360/681-4564

Sample Name:

Sample Type:

Matrix:

Batch ID:

Sample Weight (g):

Extraction Date:

Analysis Date:

Units (wet wt):

Naphthalene

C1-Naphthalenes

C2-Naphthalenes

C3-Naphthalenes

C4-Naphthalenes

Biphenyl

Acenaphthylene

Acenaphthene

Fluorene

C1-Fluorenes

C2-Fluorenes

C3-Fluorenes

Anthracene

Phenanthrene

C1-Phenanthrenes/Anthracenes

C2-Phenanthrenes/Anthracenes

C3-Phenanthrenes/Anthracenes

C4-Phenanthrenes/Anthracenes

Dibenzothiophene

C1-Dibenzothiophenes

C2-Dibenzothiophenes

C3-Dibenzothiophenes

C4-Dibenzothiophenes

Fluoranthene

Pyrene

C1-Fluoranthenes/Pyrenes

C2-Fluoranthenes/Pyrenes

C3-Fluoranthenes/Pyrenes

Benzo(a)anthracene

Chrysene

C1-Chrysenes

C2-Chrysenes

C3-Chrysenes

C4-Chrysenes

Benzo(b)fluoranthene

Benzo(k)fluoranthene

Benzo(e)pyrene

Benzo(a)pyrene

Perylene

BLK070210
$\mathrm{MB}$
Diatomaceous
earth \& Sodium
Sulfate $^{1}$
$070210 \mathrm{PAH}$
devided by $10 \mathrm{~g}$
$7 / 2 / 2010$
$8 / 12 / 2010$

BLK070610
$\mathrm{MB}$
Diatomaceous
earth \& Sodium
Sulfate ${ }^{1}$
$070610 \mathrm{PAH}$
devided by $10 \mathrm{~g}$
$7 / 6 / 2010$
$8 / 19 / 2010$
$\mathrm{ng} / \mathrm{g}$

BLK071910
MB
Diatomaceous
earth \& Sodium
Sulfate $^{1}$
$071910 \mathrm{PAH}$
devided by $10 \mathrm{~g}$
$7 / 19 / 2010$
$8 / 27 / 2010$

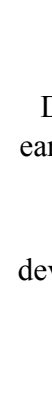

$1.82 \mathrm{U}$

$1.82 \mathrm{U}$

$3.61 \mathrm{U}$

$3.61 \mathrm{U}$

$3.61 \mathrm{U}$

$2.86 \mathrm{U}$

$1.68 \mathrm{U}$

$2.29 \mathrm{U}$

$2.75 \mathrm{U}$

$2.17 \mathrm{U}$

$2.17 \mathrm{U}$

$2.17 \mathrm{U}$

$0.42 \mathrm{U}$

$1.10 \mathrm{U}$

$1.18 \mathrm{U}$

$0.10 \mathrm{U}$

$0.10 \mathrm{U}$

$0.10 \mathrm{U}$

$0.40 \mathrm{U}$

$0.40 \mathrm{U}$

$0.40 \mathrm{U}$

$0.40 \mathrm{U}$

$0.40 \mathrm{U}$

$0.81 \mathrm{U}$

$0.35 \mathrm{U}$

$0.81 \mathrm{U}$

$0.81 \mathrm{U}$

$0.81 \mathrm{U}$

$0.54 \mathrm{U}^{*}$

$0.87 \mathrm{U}^{*}$

$0.87 \mathrm{U}^{*}$

$0.87 \mathrm{U}^{*}$

$0.87 \mathrm{U}^{*}$

$0.87 \mathrm{U}^{*}$

$0.37 \mathrm{U}^{*}$

$0.21 \mathrm{U}^{*}$

$0.22 \mathrm{U}^{*}$

$0.23 \mathrm{U}^{*}$

$0.25 \mathrm{U}$

ng/g

\subsection{6}

$1.82 \mathrm{U}$

$3.61 \mathrm{U}$

$3.61 \mathrm{U}$

$3.61 \mathrm{U}$

$2.86 \mathrm{U}$

$1.68 \mathrm{U}$

$2.29 \mathrm{U}$

$2.75 \mathrm{U}$

$2.17 \mathrm{U}$

$2.17 \mathrm{U}$

$2.17 \mathrm{U}$

$0.42 \mathrm{U}$

$1.10 \mathrm{U}$

$1.18 \mathrm{U}$

$0.10 \mathrm{U}$

$0.10 \mathrm{U}$

$0.10 \mathrm{U}$

$0.40 \mathrm{U}$

$0.40 \mathrm{U}$

$0.40 \mathrm{U}$

$0.40 \mathrm{U}$

$0.40 \mathrm{U}$

$0.81 \mathrm{U}$

$0.35 \mathrm{U}$

$0.81 \mathrm{U}$

$0.81 \mathrm{U}$

$0.81 \mathrm{U}$

$0.54 \mathrm{U}$

$0.87 \mathrm{U}$

$0.87 \mathrm{U}$

$0.87 \mathrm{U}$

$0.87 \mathrm{U}$

$0.87 \mathrm{U}$

$0.37 \mathrm{U}$

$0.21 \mathrm{U}$

$0.22 \mathrm{U}$

$0.23 \mathrm{U}$

$0.25 \mathrm{U}$
BLK072910

MB

Diatomaceous

earth \& Sodium

Sulfate $^{1}$

072910PAH

devided by $10 \mathrm{~g}$

$7 / 29 / 2010$

$8 / 7 / 2010$

$\mathrm{ng} / \mathrm{g}$

4.36

$1.82 \mathrm{U}$

$3.61 \mathrm{U}$

$3.61 \mathrm{U}$

$3.61 \mathrm{U}$

$2.86 \mathrm{U}$

$1.68 \mathrm{U}$

$2.29 \mathrm{U}$

$2.75 \mathrm{U}$

$2.17 \mathrm{U}$

$2.17 \mathrm{U}$

$2.17 \mathrm{U}$

$0.42 \mathrm{U}$

$1.10 \mathrm{U}$

$1.18 \mathrm{U}$

$0.10 \mathrm{U}$

$0.10 \mathrm{U}$

$0.10 \mathrm{U}$

$0.40 \mathrm{U}$

$0.40 \mathrm{U}$

$0.40 \mathrm{U}$

$0.40 \mathrm{U}$

$0.40 \mathrm{U}$

$0.81 \mathrm{U}$

$0.35 \mathrm{U}$

$0.81 \mathrm{U}$

$0.81 \mathrm{U}$

$0.81 \mathrm{U}$

$0.54 \mathrm{U}$

$0.87 \mathrm{U}$

$0.87 \mathrm{U}$

$0.87 \mathrm{U}$

$0.87 \mathrm{U}$

$0.87 \mathrm{U}$

$0.37 \mathrm{U}$

$0.21 \mathrm{U}$

$0.22 \mathrm{U}$

$0.23 \mathrm{U}$

$0.25 \mathrm{U}$
BLK080610 MB

Diatomaceous earth \& Sodium

Sulfate $^{1}$

080610PAH

devided by $10 \mathrm{~g}$

$8 / 6 / 2010$

$8 / 11 / 2010$

$\mathrm{ng} / \mathrm{g}$

$0.70 \mathrm{U}$

$1.82 \mathrm{U}$

$3.61 \mathrm{U}$

$3.61 \mathrm{U}$

$3.61 \mathrm{U}$

$2.86 \mathrm{U}$

$1.68 \mathrm{U}$

$2.29 \mathrm{U}$

$2.75 \mathrm{U}$

$2.17 \mathrm{U}$

$2.17 \mathrm{U}$

$2.17 \mathrm{U}$

$0.42 \mathrm{U}$

$1.10 \mathrm{U}$

$1.18 \mathrm{U}$

$0.10 \mathrm{U}$

$0.10 \mathrm{U}$

$0.10 \mathrm{U}$

$0.40 \mathrm{U}$

$0.40 \mathrm{U}$

$0.40 \mathrm{U}$

$0.40 \mathrm{U}$

$0.40 \mathrm{U}$

$0.81 \mathrm{U}$

$0.35 \mathrm{U}$

$0.81 \mathrm{U}$

$0.81 \mathrm{U}$

$0.81 \mathrm{U}$

$0.54 \mathrm{U}$

$0.87 \mathrm{U}$

$0.87 \mathrm{U}$

$0.87 \mathrm{U}$

$0.87 \mathrm{U}$

$0.87 \mathrm{U}$

$0.37 \mathrm{U}$

$0.21 \mathrm{U}$

$0.22 \mathrm{U}$

$0.23 \mathrm{U}$

$0.25 \mathrm{U}$ 
BATTELLE MARINE SCIENCE LABORATORIES

1529 West Sequim Bay Road

Sequim, Washington 98382-9099

360/681-4564

Sample Name:

Sample Type:

Matrix:

Batch ID:

Sample Weight (g):

Extraction Date:

Analysis Date:

Units (wet wt):

Indeno(1,2,3-cd)pyrene

Dibenz(a,h)anthracene

$\operatorname{Benzo}(\mathrm{g}, \mathrm{h}, \mathrm{i})$ perylene

\section{SURROGATE RECOVERIES (\%Rec)}

d8-Naphthalene

d10-Acenaphthene

d10-Phenanthrene

d12-Chrysene

d12-Perylene

\footnotetext{
${ }^{1}$ Diatomaceous earth \& Sodium Sulfate are the drying and dispersing agents (mixed with sample) used in the extraction
}

\section{Regional Mussel Watch - AMB02 \\ ENVVEST 2010 \\ PAHs in Indigenous Mussels \\ UNITS: $\mathrm{ng} / \mathrm{g}$ wet wt. \\ QC Summary 2010 Mussel Study}

BLK070210

$\mathrm{MB}$

Diatomaceous earth \& Sodium

Sulfate $^{1}$

070210PAH

devided by $10 \mathrm{~g}$

$7 / 2 / 2010$

$8 / 12 / 2010$

$\mathrm{ng} / \mathrm{g}$
BLK070610

$\mathrm{MB}$

Diatomaceous earth \& Sodium

Sulfate $^{1}$

070610PAH

devided by $10 \mathrm{~g}$

$7 / 6 / 2010$

$8 / 19 / 2010$

$\mathrm{ng} / \mathrm{g}$
$0.14 \mathrm{U}^{*}$

$0.06 \mathrm{U}^{*}$

$0.22 \mathrm{U}^{*}$

$88 \%$
$84 \%$
$81 \%$
$125 \%$ \#
$110 \%$

$125 \%$
BLK071910

MB

Diatomaceous

earth \& Sodium

Sulfate $^{1}$

071910PAH

devided by $10 \mathrm{~g}$

$7 / 19 / 2010$

$8 / 27 / 2010$

$\mathrm{ng} / \mathrm{g}$

$0.14 \mathrm{U}$

$0.06 \mathrm{U}$

$0.22 \mathrm{U}$

$41 \%$
$58 \%$
$72 \%$
$88 \%$
$84 \%$
BLK072910

MB

Diatomaceous

earth \& Sodium

Sulfate $^{1}$

072910PAH

devided by $10 \mathrm{~g}$

$7 / 29 / 2010$

$8 / 7 / 2010$

$\mathrm{ng} / \mathrm{g}$

$0.14 \mathrm{U}$

$0.06 \mathrm{U}$

$0.22 \mathrm{U}$

$98 \%$

$76 \%$

$78 \%$

$70 \%$

$67 \%$
BLK080610

MB

Diatomaceous

earth \& Sodium

Sulfate $^{1}$

080610PAH

devided by $10 \mathrm{~g}$

$8 / 6 / 2010$

$8 / 11 / 2010$

$\mathrm{ng} / \mathrm{g}$

$0.14 \mathrm{U}$

$0.06 \mathrm{U}$

$0.22 \mathrm{U}$
$93 \%$

$96 \%$

$88 \%$
$97 \%$

$112 \%$

$82 \%$

$103 \%$

$95 \%$ 
BATTELLE MARINE SCIENCE LABORATOF

1529 West Sequim Bay Road

Sequim, Washington 98382-9099

360/681-4564

Sample Name:

Sample Type:

Matrix:

Batch ID:

Sample Weight (g):

Extraction Date:

Analysis Date:

Units (wet wt): $\begin{array}{rr} & \text { Spike } \\ 3106-200 & \text { Amt } \\ \text { Reg_Sample } & \text { Standards }\end{array}$
2010 Regional Mussel Watch - AMB02

ENVVEST 2010

PAHs in Indigenous Mussels

UNITS: $n g / g$ wet wt.

QC Summary 2010 Mussel Study

\begin{tabular}{|c|c|c|c|c|c|c|c|}
\hline Naphthalene & $5.73 \mathrm{~B}$ & 100 & $22.79 \mathrm{~B}$ & $182 \% \mathrm{c}$ & 100 & 15.65 & $104 \%$ \\
\hline C1-Naphthalenes & $3.71 \mathrm{~B}$ & & & & & & \\
\hline C2-Naphthalenes & 9.19 & & & & & & \\
\hline C3-Naphthalenes & 7.69 & & & & & & \\
\hline C4-Naphthalenes & 4.72 & & & & & & \\
\hline Biphenyl & $2.86 \mathrm{U}$ & 100 & 12.90 & $138 \% \&$ & 100 & 11.13 & $116 \%$ \\
\hline Acenaphthylene & $1.68 \mathrm{U}$ & 100 & 12.65 & $135 \% \&$ & 100 & 11.01 & $115 \%$ \\
\hline Acenaphthene & $3.48 \quad 100$ & & 14.82 & $121 \% \&$ & 100 & 13.84 & $108 \%$ \\
\hline Fluorene & $3.98 \quad 100$ & & 17.24 & $142 \% \&$ & 100 & 11.34 & $77 \%$ \\
\hline C1-Fluorenes & 16.40 & & & & & & \\
\hline C2-Fluorenes & $2.17 \mathrm{U}$ & & & & & & \\
\hline C3-Fluorenes & $2.17 \mathrm{U}$ & & & & & & \\
\hline Anthracene & $5.49 \quad 100$ & & 12.85 & $79 \%$ & 100 & 12.09 & $69 \%$ \\
\hline Phenanthrene & 17.36100 & & 26.08 & $93 \%$ & 100 & 25.94 & $90 \%$ \\
\hline C1-Phenanthrenes/Anthracenes & 63.78 & & & & & & \\
\hline C2-Phenanthrenes/Anthracenes & 13.68 & & & & & & \\
\hline C3-Phenanthrenes/Anthracenes & 16.04 & & & & & & \\
\hline C4-Phenanthrenes/Anthracenes & $0.10 \mathrm{U}$ & & & & & & \\
\hline Dibenzothiophene & $0.68 \mathrm{~J}$ & 100 & 9.36 & $93 \%$ & 100 & 9.16 & $88 \%$ \\
\hline C1-Dibenzothiophenes & $1.65 \mathrm{~J}$ & & & & & & \\
\hline C2-Dibenzothiophenes & 3.70 & & & & & & \\
\hline C3-Dibenzothiophenes & $0.40 \mathrm{U}$ & & & & & & \\
\hline C4-Dibenzothiophenes & 3.70 & & & & & & \\
\hline Fluoranthene & 17.36100 & & 26.01 & $92 \%$ & 100 & 29.20 & $124 \%$ \\
\hline Pyrene & 8.62100 & & 16.74 & $87 \%$ & 100 & 19.06 & $109 \%$ \\
\hline C1-Fluoranthenes/Pyrenes & 11.35 & & & & & & \\
\hline C2-Fluoranthenes/Pyrenes & 3.23 & & & & & & \\
\hline C3-Fluoranthenes/Pyrenes & $0.81 \mathrm{U}$ & & & & & & \\
\hline Benzo(a)anthracene & 5.93100 & & 15.83 & $106 \%$ & 100 & 15.97 & $105 \%$ \\
\hline Chrysene & 13.81100 & & 20.87 & $75 \%$ & 100 & 21.64 & $82 \%$ \\
\hline C1-Chrysenes & 3.35 & & & & & & \\
\hline C2-Chrysenes & $0.87 \mathrm{U}$ & & & & & & \\
\hline C3-Chrysenes & $0.87 \mathrm{U}$ & & & & & & \\
\hline C4-Chrysenes & $0.87 \mathrm{U}$ & & & & & & \\
\hline Benzo(b)fluoranthene & $6.42 \quad 100$ & & 16.15 & $104 \%$ & 100 & 15.90 & $99 \%$ \\
\hline Benzo(k)fluoranthene & $2.85 \quad 100$ & & 12.36 & $102 \%$ & 100 & 12.02 & $96 \%$ \\
\hline Benzo(e)pyrene & $3.70 \quad 100$ & & 10.66 & $74 \%$ & 100 & 10.61 & $72 \%$ \\
\hline Benzo(a)pyrene & $2.13 \quad 100$ & & 10.74 & $92 \%$ & 100 & 10.61 & $88 \%$ \\
\hline Perylene & $1.70 \mathrm{~J}$ & 100 & 11.34 & $103 \%$ & 100 & 10.62 & $93 \%$ \\
\hline
\end{tabular}


BATTELLE MARINE SCIENCE LABORATOF

1529 West Sequim Bay Road

Sequim, Washington 98382-9099

360/681-4564

Sample Name:

Sample Type:

Matrix:

Batch ID:

Sample Weight (g):

Extraction Date:

Analysis Date:

Units (wet wt):

Indeno(1,2,3-cd)pyrene

Dibenz(a,h)anthracene

$\operatorname{Benzo}(\mathrm{g}, \mathrm{h}, \mathrm{i})$ perylene

\section{SURROGATE RECOVERIES (\%Rec)}

d8-Naphthalene

d10-Acenaphthene

d10-Phenanthrene

d12-Chrysene

d12-Perylene

\footnotetext{
${ }^{1}$ Diatomaceous earth \& Sodium Sulfate are the drying and dispersing agents (mixed with sample) used in the extraction
}

$$
\text { 3106-200 }
$$

Reg_Sample

Spike
Amt
Standards

3106-

200SPK1

MS

2010 Regional Mussel Watch - AMB02

ENVVEST 2010

PAHs in Indigenous Mussels

UNITS: $\mathrm{ng} / \mathrm{g}$ wet wt.

QC Summary 2010 Mussel Study 
BATTELLE MARINE SCIENCE LABORATOF

1529 West Sequim Bay Road

Sequim, Washington 98382-9099

360/681-4564

Sample Name:

Sample Type:

Matrix:

Batch ID:

Sample Weight (g):

Extraction Date:

Analysis Date:

Units (wet wt):

Naphthalene

C1-Naphthalenes

C2-Naphthalenes

C3-Naphthalenes

C4-Naphthalenes

Biphenyl

Acenaphthylene

Acenaphthene

Fluorene

C1-Fluorenes

C2-Fluorenes

C3-Fluorenes

Anthracene

Phenanthrene

C1-Phenanthrenes/Anthracenes

C2-Phenanthrenes/Anthracenes

C3-Phenanthrenes/Anthracenes

C4-Phenanthrenes/Anthracenes

Dibenzothiophene

C1-Dibenzothiophenes

C2-Dibenzothiophenes

C3-Dibenzothiophenes

C4-Dibenzothiophenes

Fluoranthene

Pyrene

C1-Fluoranthenes/Pyrenes

C2-Fluoranthenes/Pyrenes

C3-Fluoranthenes/Pyrenes

Benzo(a)anthracene

Chrysene

C1-Chrysenes

C2-Chrysenes

C3-Chrysenes

C4-Chrysenes

Benzo(b)fluoranthene

Benzo(k)fluoranthene

Benzo(e)pyrene

Benzo(a)pyrene

Perylene
2010 Regional Mussel Watch - AMB02

ENVVEST 2010

PAHs in Indigenous Mussels

UNITS: $n g / g$ wet wt.

QC Summary 2010 Mussel Study

$\begin{array}{rrrr}\text { SIPOM 3106- } & \text { SIPOM 3106 } & \text { SIWP 3106- } & \text { SIWP 3106- } \\ 199 & 199 \mathrm{~B} & 204 & \text { 204B } \\ \text { Reg_Sample } & \text { Duplicate } & \text { Reg_Sample } & \text { Duplicate }\end{array}$

\begin{tabular}{|c|c|c|c|}
\hline TISSUE & TISSUE & TISSUE & TISSUI \\
\hline 070210PAH & 070210PAH & 071910РAH & 072910P \\
\hline 11.45 & 11.44 & 11.59 & 1 \\
\hline $7 / 2 / 2010$ & $7 / 2 / 2010$ & $7 / 19 / 2010$ & $7 / 29 / 201$ \\
\hline $8 / 12 / 2010$ & $8 / 12 / 2010$ & $8 / 12 / 2010$ & $8 / 12 / 20$ \\
\hline $\mathrm{ng} / \mathrm{g}$ & $\mathrm{ng} / \mathrm{g}$ & $\mathrm{ng} / \mathrm{g}$ & \\
\hline
\end{tabular}

$\begin{array}{lll}5.00 \mathrm{~B} & 9.82 & \\ 5.44 \mathrm{~B} & 6.53 & 3.32\end{array}$

$65 \% \mathrm{c}$

11.3513 .26

$19.36 \quad 21.00$

$21.55 \quad 24.18$

$2.86 \mathrm{U}$

$1.68 \mathrm{U}$

$2.29 \mathrm{U}$

$2.86 \mathrm{U}$

$1.72 \mathrm{~J}$

$2.29 \mathrm{U}$

$2.75 \mathrm{U}$

$16.61 \quad 14.40$

$2.17 \mathrm{U}$

$2.82 \quad 2.75$

$2.17 \mathrm{U}$

3.293 .32

$\begin{array}{ll}17.95 & 19.28\end{array}$

$50.47 \quad 55.97$

$62.35 \quad 64.50$

$39.94 \quad 40.67$

33.8934 .56

$1.08 \mathrm{~J}$

$3.30 \quad 3.57$

9.669 .36

$10.68 \quad 10.44$

$8.50 \quad 7.61$

\&

$2.17 \mathrm{U}$

$2.17 \mathrm{U}$

$1 \%$

$7 \%$

$38.00 \quad 39.68$

$24.90 \quad 23.74$

$18.50 \quad 18.95$

$9.05 \quad 8.44$

$0.81 \mathrm{U}$

$\begin{array}{ll}7.10 & 7.22\end{array}$

$16.44 \quad 16.72$

$\begin{array}{ll}5.96 & 6.38\end{array}$

6.726 .04

$0.87 \mathrm{U}$

$0.87 \mathrm{U}$

$0.87 \mathrm{U}$

$8.35 \quad 8.40$

$3.39 \quad 3.31$

6.296 .28

$1.64 \mathrm{~J}$

$0.87 \mathrm{U}$

$0.81 \mathrm{U}$

$4 \%$

$5 \%$

$1 \%$

$7.41 \mathrm{~B}$

$7.41 \mathrm{~B}$

9.21

6.70

5.09

$2.86 \mathrm{U}$

$1.68 \mathrm{U}$

$2.29 \mathrm{U}$

U

16.12

$2.17 \mathrm{U}$

$2.17 \mathrm{U}$

$0.99 \mathrm{~J}$

5.49

5.15

13.31

81.93

$0.10 \mathrm{U}$

$0.40 \mathrm{U}$

$0.40 \mathrm{U}$

$0.40 \mathrm{U}$

$0.40 \mathrm{U}$

$0.40 \mathrm{U}$

5.98

3.27

3.42

$0.81 \mathrm{U}$

$0.81 \mathrm{U}$

$2 \%$

$2 \%$

2.19

4.91

$0.87 \mathrm{U}$

$0.87 \mathrm{U}$

$0.87 \mathrm{U}$

$0.87 \mathrm{U}$

2.18

$0.86 \mathrm{~J}$

$1.43 \mathrm{~J}$

$1.58 \mathrm{~J}$

$1.06 \mathrm{~J}$

$0.71 \mathrm{~J}$

$0.69 \mathrm{~J}$

$\begin{array}{ll}1 \% & 2.18 \\ 2 \% & 0.86 \mathrm{~J} \\ 0 \% & 1.43 \mathrm{~J} \\ 3 \% & 0.71 \mathrm{~J} \\ 8 \% & 0.69 \mathrm{~J}\end{array}$

$\mathrm{ng} / \mathrm{g}$

5.43

4.21

9.00

4.94

8.96

9.47

63.53

$0.46 \mathrm{~J}$

5.21

2.88

3.48

2.04

4.53

2.20

$0.91 \mathrm{~J}$

$1.40 \mathrm{~J}$

$0.65 \mathrm{~J}$

$0.69 \mathrm{~J}$

RPD

2.33 B $104 \%$ c

$1.82 \mathrm{U}$

$3.61 \mathrm{U}$

$0.61 \mathrm{U}$

$0.37 \mathrm{U}$

$0.58 \mathrm{U}$

$0.74 \mathrm{U}$

$2.17 \mathrm{U}$

$2.17 \mathrm{U}$

$0.93 \mathrm{~J}$

$0.10 \mathrm{U}$

$0.40 \mathrm{U}$

$0.40 \mathrm{U}$

$0.40 \mathrm{U}$

$0.40 \mathrm{U}$

$14 \%$

$13 \%$

$0.81 \mathrm{U}$

$0.81 \mathrm{U}$

$6 \%$

$11 \%$

$0.87 \mathrm{U}$

$0.87 \mathrm{U}$

$0.87 \mathrm{U}$

$0.87 \mathrm{U}$ 
BATTELLE MARINE SCIENCE LABORATOF

1529 West Sequim Bay Road

Sequim, Washington 98382-9099

$360 / 681-4564$

Sample Name:

Sample Type:

Matrix:

Batch ID:

Sample Weight (g):

Extraction Date:

Analysis Date:

Units (wet wt):

Indeno(1,2,3-cd)pyrene

Dibenz(a,h)anthracene

$\operatorname{Benzo}(\mathrm{g}, \mathrm{h}, \mathrm{i})$ perylene

\section{SURROGATE RECOVERIES (\%Rec)}

d8-Naphthalene

$57 \%$

$72 \%$

$93 \%$

$89 \%$

$87 \%$
2010 Regional Mussel Watch - AMB02

ENVVEST 2010

PAHs in Indigenous Mussels

UNITS: $\mathrm{ng} / \mathrm{g}$ wet wt.

QC Summary 2010 Mussel Study

$\begin{array}{rr}\text { SIPOM 3106- } & \text { SIPOM } 3106 . \\ 199 & 199 \mathrm{~B} \\ \text { Reg_Sample } & \text { Duplicate }\end{array}$

$\begin{array}{rr}\text { TISSUE } & \text { TISSUE } \\ 070210 \mathrm{PAH} & 070210 \mathrm{PAH} \\ 11.45 & 11.44 \\ 7 / 2 / 2010 & 7 / 2 / 2010 \\ 8 / 12 / 2010 & 8 / 12 / 2010 \\ \mathrm{ng} / \mathrm{g} & \mathrm{ng} / \mathrm{g}\end{array}$

$1.21 \mathrm{~J}$
$0.06 \mathrm{U}$
1.981 .64

$1.16 \mathrm{~J}$

$0.06 \mathrm{U}$

$5 \%$

$19 \%$

$49 \%$
$71 \%$
$90 \%$
$87 \%$
$81 \%$

$49 \%$

$71 \%$

$90 \%$

$81 \%$

$\begin{array}{rr}\text { SIWP 3106- } & \text { SIWP 3106- } \\ 204 & \text { 204B } \\ \text { Reg_Sample } & \text { Duplicate }\end{array}$

TISSUE

071910PAH

11.59

$7 / 19 / 2010$

$8 / 12 / 2010$

$\mathrm{ng} / \mathrm{g}$

$0.52 \mathrm{~J}$

$0.06 \mathrm{U}$

$0.74 \mathrm{~J}$

$\begin{array}{ll}47 \% & 70 \% \\ 60 \% & 75 \% \\ 88 \% & 94 \% \\ 89 \% & 93 \% \\ 84 \% & 87 \%\end{array}$

\footnotetext{
${ }^{1}$ Diatomaceous earth \& Sodium Sulfate are the drying and dispersing agents (mixed with sample) used in the extraction
} 
BATTELLE MARINE SCIENCE LABORATORIES

1529 West Sequim Bay Road

Sequim, Washington 98382-9099

360/681-4564

2010 Regional Mussel Watch - AMB02

ENVVEST 2010

PAHs in Indigenous Mussels

UNITS: ng/g wet wt.

\section{$\underline{\text { Data Qualifiers }}$}

U Not detected at or above laboratory achieved detection limit; MDL reported

$\mathrm{J}$ Analyte concentration is less that the RL, but greater than the MDL

B Analyte detected in the method blank above the RL, sample concentration $<10 \mathrm{x}$ detected blank value

$\&$ Outside Project DQO for spike recovery (40-120\%), replicate analysis $(<30 \%)$, or SRM PD $(<30 \%)$

D Results determined from dilution

c Exceeds Project DQO but meets contingency criteria

\# Outside Project DQOs for surrogate Recovery (40-120\%)

* Associated surrogate recovery exceeds DQO guidelines 


\section{QA Narratives}




\section{QA/QC NARRATIVE}

PROJECT:

PARAMETER:

LABORATORY: MATRIX:

SAMPLE CUSTODY AND PROCESSING:
Regional Mussel Watch AMB02 - 2010

Metals: silver (Ag), arsenic (As), cadmium (Cd), chromium $(\mathrm{Cr})$, copper $(\mathrm{Cu})$, mercury $(\mathrm{Hg})$, nickel $(\mathrm{Ni})$, lead $(\mathrm{Pb})$, and zinc $(\mathrm{Zn})$

Battelle Marine Sciences Laboratory, Sequim, Washington Tissues (indigenous mussels)

Mussels from 24 sampling locations were collected by the U.S. Navy following NOAA Mussel watch protocol and hand delivered to MSL on 01/09/10, 01/13/2010, $02 / 02 / 2010,02 / 03 / 2010$, and $02 / 05 / 2010$. The live mussels were stored at $-20^{\circ} \mathrm{C}$ until they were measured and shucked. The length of each mussel added to the composite sample was recorded along with the total number of specimens in each composite. The mussels were rinsed with deionized water, shucked using a ceramic knife, and composites were homogenized to an even color and consistency using a titanium blender. The composites were split into three containers: 1) polypropylene pre-cleaned vial, tarred for percent moisture determination and metals analyses, 2) $8 \mathrm{oz}$. precleaned glass jar for organic compound analyses, and 3) $8 \mathrm{oz}$. pre-cleaned glass jar for archival. The following quality control summary addresses the analyses of the 24 composite samples for trace metals. The composite samples were assigned a Battelle Central File (CF) identification number (3106). All project information was entered into Battelle's laboratory information and sample tracking system.

The following lists information on sample receipt and processing activities:

\begin{tabular}{cc}
\hline Chemistry Lab ID & $\mathbf{3 1 0 6} * \mathbf{2 1 5 - 2 3 8}$ \\
\hline Description & mussels \\
\hline Collection dates & $01 / 08 / 10,01 / 12 / 10$, and $02 / 02$ through $02 / 04 / 10$ \\
Laboratory arrival date & $01 / 09 / 10,01 / 13 / 2010,02 / 02 / 2010,02 / 03 / 2010$, and \\
Cooler temperatures, on arrival & $02 / 05 / 2010$ \\
Digestion (aqua regia) & $4 \pm 2{ }^{\circ} \mathrm{C}$ \\
CVAA Analysis Date & $04 / 14 / 10$ \\
(Hg) & $05 / 06 / 10$ \\
ICP-OES Analysis Date & \\
$\quad(\mathrm{Cr}, \mathrm{Cu}, \mathrm{Ni}, \mathrm{Zn})$ & $04 / 23 / 10$ \\
ICP-MS Analysis Date & $04 / 21 / 10$ \\
$(\mathrm{Ag}, \mathrm{As}, \mathrm{Cd}, \mathrm{Pb})$ & \\
\hline
\end{tabular}




\section{QA/QC NARRATIVE}

\begin{tabular}{|c|c|c|c|c|c|c|}
\hline Analyte & $\begin{array}{c}\text { Analytical } \\
\text { Method }\end{array}$ & $\begin{array}{l}\text { Range of } \\
\text { Recovery }\end{array}$ & $\begin{array}{c}\text { SRM } \\
\text { Accuracy } \\
\end{array}$ & $\begin{array}{c}\text { Relative } \\
\text { Precision }\end{array}$ & $\begin{array}{c}\text { MDL } \\
(\mu \mathrm{g} / \mathrm{g} \text { dry wt. })^{\mathrm{a}}\end{array}$ & $\begin{array}{c}\text { RL } \\
(\mu \mathrm{g} / \mathrm{g} \text { dry wt. })^{\mathrm{b}}\end{array}$ \\
\hline Silver & ICP-MS & $70-130 \%$ & $\leq 20 \%$ & $\leq 30 \%$ & 0.0031 & 0.010 \\
\hline Arsenic & ICP-MS & $70-130 \%$ & $\leq 20 \%$ & $\leq 30 \%$ & 0.11 & 0.35 \\
\hline Cadmium & ICP-MS & $70-130 \%$ & $\leq 20 \%$ & $\leq 30 \%$ & 0.0029 & 0.0092 \\
\hline Chromium & ICP-OES & $70-130 \%$ & $\leq 20 \%$ & $\leq 30 \%$ & 0.035 & 0.11 \\
\hline Copper & ICP-OES & $70-130 \%$ & $\leq 20 \%$ & $\leq 30 \%$ & 0.094 & 0.30 \\
\hline Mercury & CVAA & $70-130 \%$ & $\leq 20 \%$ & $\leq 30 \%$ & 0.0044 & 0.014 \\
\hline Nickel & ICP-OES & $70-130 \%$ & $\leq 20 \%$ & $\leq 30 \%$ & 0.041 & 0.13 \\
\hline Lead & ICP-MS & $70-130 \%$ & $\leq 20 \%$ & $\leq 30 \%$ & 0.0034 & 0.011 \\
\hline Zinc & ICP-OES & $70-130 \%$ & $\leq 20 \%$ & $\leq 30 \%$ & 0.018 & 0.057 \\
\hline
\end{tabular}

(a) Achieved method detection limits (MDLs) reported from the annual MDL study using seven replicates of a tissue matrix prepared and analyzed as samples.

(b) The reporting limit (RL) was determined as 3.18 times the achieved MDL.

\section{METHODS:} metals, and stored at $-80 \pm 2^{\circ} \mathrm{C}$ until lyophilization according to MSL-C-003. The samples were analyzed for nine metals including $\mathrm{Ag}, \mathrm{As}, \mathrm{Cd}, \mathrm{Cr}, \mathrm{Cu}, \mathrm{Pb}, \mathrm{Hg}, \mathrm{Ni}$, and Zn. Tissue samples were digested according to Battelle SOP MSL-I-024, Mixed Acid Tissue Digestion. An approximately 500-mg aliquot of each dried, homogeneous sample was combined with nitric and hydrochloric acids (aqua regia) in a Teflon vessel and heated in an oven at $130^{\circ} \mathrm{C}\left( \pm 10^{\circ} \mathrm{C}\right)$ for a minimum of eight hours. After heating and cooling, deionized water was added to the acid-digested tissue to achieve analysis volume and the digestates were submitted for analysis by three methods.

Digested samples were analyzed for $\mathrm{Hg}$ by cold-vapor atomic absorption spectroscopy (CVAA) according to Battelle SOP MSL-I-016, Total Mercury in Tissues and Sediments by Cold Vapor Atomic Absorption, which is based on EPA Method 245.6, Determination of Mercury in Tissue by Cold Vapor Atomic Absorption Spectrometry.

Digested samples were analyzed for $\mathrm{Cr}, \mathrm{Cu}, \mathrm{Ni}$, and $\mathrm{Zn}$ using inductively coupled plasma optical emissions spectroscopy (ICP-OES) according to Battelle SOP MSL-I033, Determination of Elements in Aqueous and Digestate Samples by ICP-OES. This procedure is based on two methods modified and adapted for analysis of low level samples: EPA Method 6010B and 200.7.

Digested samples were analyzed for $\mathrm{Ag}, \mathrm{As}, \mathrm{Cd}$, and $\mathrm{Pb}$ using inductively coupled plasma-mass spectrometry (ICP-MS) according to Battelle SOP MSL-I-022, Determination of Elements in Aqueous and Digestate Samples by ICP/MS. This procedure is based on two methods modified and adapted for analysis of solid sample digestates: EPA Method 1638, Determination of Trace Elements in Ambient Waters by Inductively Coupled Plasma-Mass Spectrometry and EPA Method 200.8, Determination of Trace Elements in Water and Wastes by Inductively Coupled Plasma - Mass Spectrometry.

All results were determined and reported in units of $\mu \mathrm{g} / \mathrm{g}$ on a dry-weight basis and converted to $\mu \mathrm{g} / \mathrm{g}$ wet-weight. The formula for this conversion is dry-weight concentration * (sample percent dry weight/100). 


\section{QA/QC NARRATIVE}

HOLDING TIMES: The EPA Method 1631 Appendix A provides a holding time for $\mathrm{Hg}$ in frozen tissue samples of one year from collection to analysis. This holding time was achieved for all metals.

DETECTION LIMITS AND DATA QUALIFIERS:

METHOD BLANKS:

LABORATORY CONTROL SAMPLE/BLANK SPIKE ACCURACY:

MATRIX SPIKE ACCURACY:

REPLICATE PRECISION:

STANDARD REFERENCE MATERIAL ACCURACY:
The MSL standard operating procedure for the determination of method detection limits (MDLs) is MSL-Q-007-08. This procedure describes the determination of MDLs, instrument detection limits (IDLs), reporting limits (RLs), and quantitation limits (QLs) as described in the Federal Register (40 CFR Part 136, Appendix B). The reporting limit (RL) is defined as 3.18 times the MDL for the matrix of interest and the instrument. The 3.18 value is based on the Student's t value for 7-10 replicates analyzed in our MDL study. The 40 CFR Part 136, Appendix B uses at least 7 replicates of the matrix, digested as independent samples, and then analyzed by the instrument of interest. The standard deviation is then multiplied by the student's $t$ value for $n-1$ replicates. Sample concentrations were evaluated and flagged to the following criteria:

$\mathrm{U}$ Analyte not detected at or above the MDL, MDL reported

J Analyte detected above the MDL, but less than the RL

$\mathrm{N}$ Spiked sample recovery outside QC criterion of $70-130 \%$

\& Accuracy result outside QC criterion of $\leq 20 \%$ PD

* Precision result outside QC criterion of $<30 \%$

B Analyte detected in the method blank $>$ RL and sample concentration $<10$ times detected blank value

c Exceeds data quality objective but meets contingency criterion

Method blanks were analyzed at a minimum frequency of one each per 20 field samples or analytical batch. Two method blanks were analyzed with the samples for all metals. The concentrations were less than RL for all metals.

Blank spikes/laboratory control samples (LCS) were analyzed at a frequency of one each per 20 field samples. The LCS recoveries were within the QC acceptance criterion of $70 \%$ to $130 \%$ recovery.

Matrix spikes and matrix spike duplicates were analyzed at a frequency of one each per 20 field samples. Matrix spike recoveries were within the QC acceptance criterion of $70 \%$ to $130 \%$ recovery for all metals.

Analytical precision was evaluated using two methods: 1) laboratory duplicate and 2) duplicate matrix spikes. Replicate analyses were performed at a frequency of one set per 20 field samples. Precision of replicate analyses was expressed as the relative percent difference (RPD) of replicate results. The RPDs for both the laboratory duplicates and duplicate matrix spikes were within the QC limits of $\leq 30 \%$ for all metals.

The SRM accuracy was expressed as the percent difference (PD) between the measured and certified SRM concentrations. Recovery of a particular analyte exceeded QC criteria if the PD exceeded 20\% PD. The SRM 1566b Oyster Tissue was analyzed at a frequency of one per 20 field samples. The recoveries for certified or referenced metals were within the QC acceptance criterion for all metals. 


\section{PCB - Tissue QA/QC SUMMARY}

\begin{tabular}{ll}
\hline PROJECT: & ENVVEST Regional Mussel Watch AMB02-2010 \\
PARAMETER: & Polychlorinated Biphenyls (PCBs) \\
LABORATORY: & Battelle Marine Sciences Laboratory, Sequim, Washington \\
MATRIX: & Tissues (indigenous mussels) \\
SAMPLE CUSTODY: & Mussels from 24 sampling locations were collected by the U.S. Navy following \\
& NOAA Mussel watch protocol and hand delivered to MSL on $01 / 09 / 10,01 / 13 / 2010$, \\
& $02 / 02 / 2010,02 / 03 / 2010$, and $02 / 05 / 2010$. The live mussels were stored at $-20^{\circ} \mathrm{C}$ until \\
& they were measured and shucked. The length of each mussel added to the composite \\
& sample was recorded along with the total number of specimens in each composite. The \\
& mussels were rinsed with deionized water, shucked using a ceramic knife, and \\
& composites were homogenized to an even color and consistency using a titanium \\
& blender. The composites were split into three containers: 1 ) polypropylene pre-cleaned \\
& vial, tarred for percent moisture determination and metals analyses, 2 ) 8 oz. pre- \\
& cleaned glass jar for organic compound analyses, and 3) 8 oz. pre-cleaned glass jar for \\
& archival. The following quality control summary addresses the analyses of the 24 \\
& composite samples for PCBs. The composite samples were assigned a Battelle Central \\
& File (CF) identification number (3106). All project information was entered into \\
& Battelle's laboratory information and sample tracking system.
\end{tabular}

\section{QA/QC DATA QUALITY OBJECTIVES:}

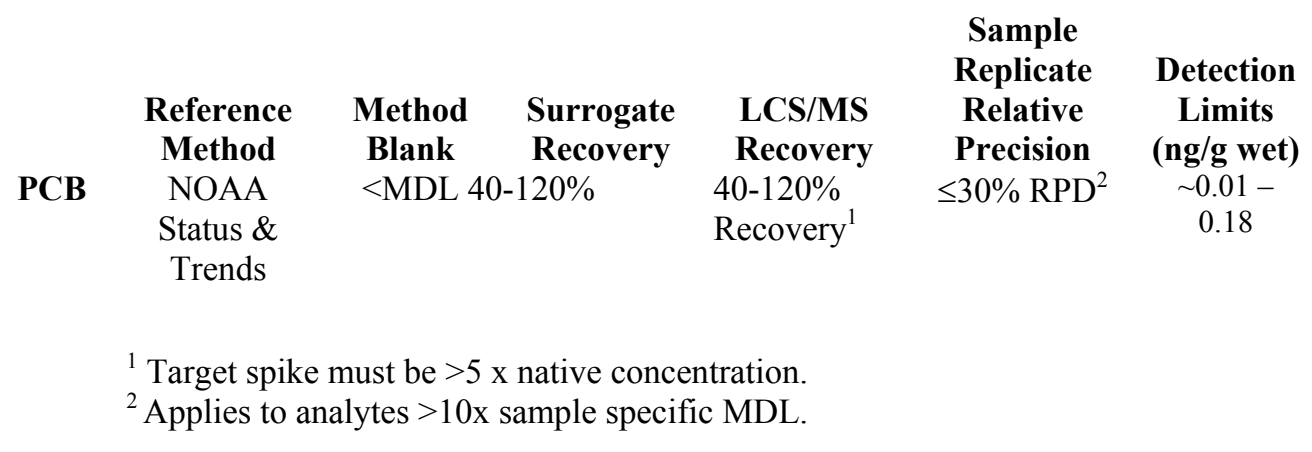

METHOD: $\quad$ Tissue samples were extracted for PCB following general National Atmospheric and Oceanic Administration (NOAA) National Status and Trends (NS\&T) methods. Approximately $10 \mathrm{~g}$ of tissue was mixed with anhydrous sodium sulfate, spiked with surrogates and extracted with methylene chloride using accelerated solvent extractor (ASE). The extract was concentrated, and processed through alumina cleanup column, concentrated, and further purified by the florisil cleanup column. The extract after florisil cleanup was concentrated and fortified with internal standard (IS), and submitted for analysis. Extracts intended for PCB analyses were analyzed using gas chromatography with electron capture detection (GC/ECD), following Battelle SOP O-016. Sample data were quantified by the method of internal standards using the surrogate compounds. 


\section{PCB - Tissue QA/QC SUMMARY}

HOLDING Tissue samples were stored frozen until sample preparation. Samples were prepared for TIMES: $\quad$ analyses in five analytical batches and were extracted within the 1-year holding time for sample collection and analyzed within 40 days of extraction.

\begin{tabular}{lcc} 
Batch & Extraction Date & Analysis Date \\
\hline 070210PCB & $7 / 2 / 2010$ & $7 / 8 / 2010$ \\
070610PCB & $7 / 6 / 2010$ & $8 / 3 / 2010$ \\
071910PCB & $7 / 19 / 2010$ & $8 / 4 / 2010$ \\
072910PCB & $7 / 29 / 2010$ & $8 / 6 / 2010$ \\
080610PCB & $8 / 6 / 2010$ & $8 / 10 / 2010$
\end{tabular}

BLANKS: $\quad$ A procedural blank (MB) was prepared with each analytical batch. The blank was analyzed to ensure the sample extraction and analysis methods were free of contamination.

Exceedences - No exceedences noted.

Comments - None.

MATRIX Two matrix spikes (MS) were prepared. The percent recoveries of PCB were calculated to SPIKES: measure data quality in terms of accuracy.

Exceedences - One exceedences noted.

Comments - In one matrix spike sample, the recovery of PCB 77 (31\%) is slightly lower than the laboratory control limits $(40-120 \%)$.

Duplicate Two duplicate samples (DUPs) were prepared. The relative percent differences (RPD) of Samples: PCB were calculated to measure data quality in terms of precision.

Exceedences - No exceedences noted.

Comments - None.

SURROGATES Three surrogate compounds were added prior to extraction, including PCB 30, PCB 65, and PCB 198. The recovery of each surrogate compound was calculated to measure data quality in terms of accuracy (extraction efficiency).

Exceedences - No exceedences noted.

Comments - None. 


\section{PAH - Tissue QA/QC SUMMARY}

\begin{tabular}{ll}
\hline PROJECT: & ENVVEST Regional Mussel Watch AMB02-2010 \\
PARAMETER: & Polycyclic Aromatic Hydrocarbons (PAHs) \\
LABORATORY: & Battelle Marine Sciences Laboratory, Sequim, Washington \\
MATRIX: & Tissues (indigenous mussels) \\
SAMPLE CUSTODY: & Mussels from 24 sampling locations were collected by the U.S. Navy following \\
& NOAA Mussel watch protocol and hand delivered to MSL on $01 / 09 / 10,01 / 13 / 2010$, \\
& $02 / 02 / 2010,02 / 03 / 2010$, and $02 / 05 / 2010$. The live mussels were stored at $-20^{\circ} \mathrm{C}$ until \\
& they were measured and shucked. The length of each mussel added to the composite \\
& sample was recorded along with the total number of specimens in each composite. The \\
& mussels were rinsed with deionized water, shucked using a ceramic knife, and \\
& composites were homogenized to an even color and consistency using a titanium \\
& blender. The composites were split into three containers: 1 ) polypropylene pre-cleaned \\
& vial, tarred for percent moisture determination and metals analyses, 2 ) 8 oz. pre- \\
& cleaned glass jar for organic compound analyses, and 3) 8 oz. pre-cleaned glass jar for \\
& archival. The following quality control summary addresses the analyses of the 24 \\
& composite samples for parent and selected akylated/methylated. The composite \\
& samples were assigned a Battelle Central File (CF) identification number (3106). All \\
project information was entered into Battelle's laboratory information and sample \\
tracking system.
\end{tabular}

\section{QA/QC DATA QUALITY OBJECTIVES:}

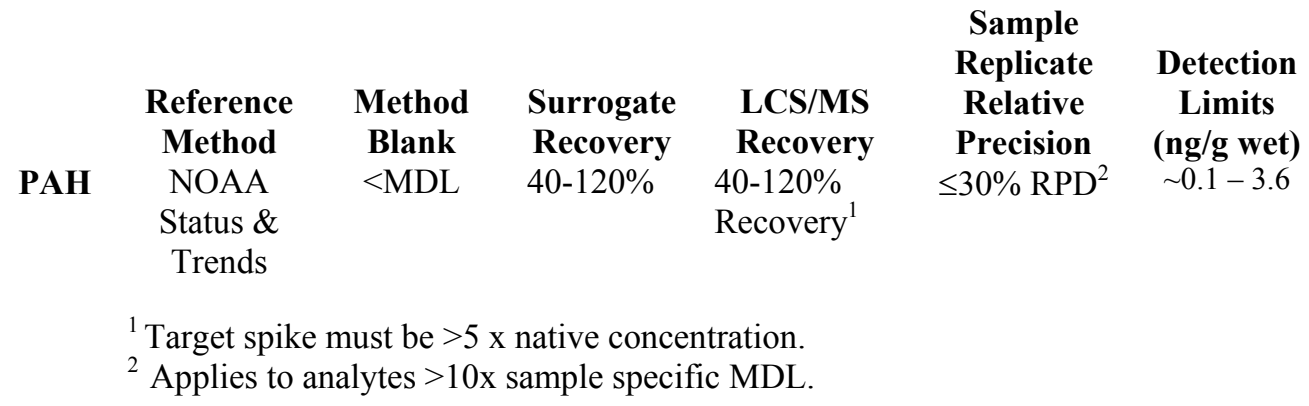

METHOD: Tissue samples were extracted for PAHs following general National Atmospheric and Oceanic Administration (NOAA) National Status and Trends (NS\&T) methods. Approximately $10 \mathrm{~g}$ of tissue was mixed with anhydrous sodium sulfate, spiked with surrogates and extracted with methylene chloride using accelerated solvent extractor (ASE). The extract was concentrated, and processed through an alumina cleanup column, concentrated, and fortified with internal standard (IS), and submitted for analysis. Extracts intended for PAH analyses were analyzed using gas chromatography mass spectrometry (GC/MS), following Battelle SOP O-015. Sample data were quantified by the method of internal standards using the surrogate compounds. 


\section{PAH - Tissue QA/QC SUMMARY}

HOLDING TIMES:

BLANKS:

MATRIX SPIKES:

Duplicate Samples:
Tissue samples were stored frozen until sample preparation. Samples were prepared for analyses in five analytical batches and were extracted within the 1-year holding time for sample collection and analyzed within 40 days of extraction.

\begin{tabular}{lcc} 
Batch & Extraction Date & Analysis Date \\
\hline 070210PAH & $7 / 2 / 2010$ & $8 / 12 / 2010$ \\
070610PAH & $7 / 6 / 2010$ & $8 / 19 / 2010^{*}$ \\
071910PAH & $7 / 19 / 2010$ & $8 / 12 / 2010$ \\
072910PAH & $7 / 29 / 2010$ & $8 / 7 / 2010$ \\
080610PAH & $8 / 6 / 2010$ & $8 / 11 / 2010$
\end{tabular}

* Initial analysis occurred 7/9/2010, however, reported concentrations for this batch are outside of the 40 days due to instrument problem. The extracts were stored in the $-20^{\circ} \mathrm{C}$ freezer before re-analysis.

A procedural blank (MB) was prepared with each analytical batch. The blank was analyzed to ensure the sample extraction and analyses methods were free of contamination.

Exceedences - One of five blanks exceeded the reporting limit (RL) for naphthalene; "B" qualifiers applied.

Comments - Naphthalene and C1-Naphthalene were detected in some blanks. "B" qualifiers were applied to these concentration when indicating the level detected in the samples were $<10 \mathrm{x}$ the blank concentration. Minimal data impact. No further corrective action taken.

Two matrix spikes (MS) were prepared. The percent recoveries of PAHs were calculated to measure data quality in terms of accuracy.

Exceedences - Six recovery exceedences were noted.

Comments - In two matrix spikes, six spike recoveries were not within the laboratory control limits $(40-120 \%)$. Those six compounds with recoveries great than $120 \%$ are naphthalene, biphenyl, acenaphthylene, acenaphthene, fluorene, and fluoranthene. However, the concentration spiked was not $>10$ times the concentration in the background material and therefore was not appropriate for data quality assessment.

Two duplicate samples (DUPs) were prepared. The relative percent differences (RPD) of parent PAHs were calculated to measure data quality in terms of precision.

Exceedences - Two RPD exceedences noted.

Comments - In two duplicate samples, the two observed RPDs were not within the laboratory control limits $(\leq 30 \%)$ both are naphthalene. All other PAHs with concentrations higher than the reporting limit have relatively narrow RPD (0-19\%). Naphthalene has been detected in the associated method blanks (MB) and thus may be a factor in the duplicate precision of this compound. However, the concentration of naphthalene in these samples are not $>10$ times the MDL.

SURROGATES Five surrogate compounds were added prior to extraction, including naphthalene-d8, acenaphthene-d10, phenanthrene-d10, chrysene-d12, and perylene-d12. The recovery of each surrogate compound was calculated to measure data quality in terms of accuracy (extraction efficiency).

Exceedences - Five exceedences noted.

Comments - Four Naphthalene-d8 (29\%-38\%) are slightly lower than laboratory control limits $(40-120 \%)$. 
PROJECT:

PARAMETER:

LABORATORY:

MATRIX:

SAMPLE CUSTODY AND PROCESSING:
ENVVEST Ambient Monitoring, AMB02 Regional Mussel Watch

Stable Isotopes of Carbon $\left(\delta^{13} \mathrm{C}\right)$ and nitrogen $\left(\delta^{15} \mathrm{~N}\right)$

Dr. Jay Brandes, Skidaway Institute for Oceanography

Indigenous Mussels

Mussels from 24 sampling locations were collected by the U.S. Navy following

NOAA Mussel watch protocol and hand delivered to MSL on 01/09/10, 01/13/2010, $02 / 02 / 2010,02 / 03 / 2010$, and $02 / 05 / 2010$. The live mussels were stored at $-20^{\circ} \mathrm{C}$ until they were measured and shucked. The length of each mussel added to the composite sample was recorded along with the total number of specimens in each composite. The mussels were rinsed with deionized water, shucked using a ceramic knife, and composites were homogenized to an even color and consistency using a titanium blender. The composites were split into three containers: 1) polypropylene pre-cleaned vial, tarred for percent moisture determination and metals analyses, 2) $8 \mathrm{oz}$. precleaned glass jar for organic compound analyses, and 3) $8 \mathrm{oz}$. pre-cleaned glass jar for archival. The following quality control summary addresses the analyses of the 24 composite samples for trace metals. The composite samples were assigned a Battelle Central File (CF) identification number (3106). All project information was entered into Battelle's laboratory information and sample tracking system. Homogenized, lyophilized tissue aliquots were sent to Skidaway Institute of Oceanography.

The following lists information on sample receipt and processing activities:

\begin{tabular}{ll}
\hline MSL Lab ID & See Table \\
Collection dates & $01 / 08 / 10,01 / 12 / 10$, and $02 / 02$ through \\
& $02 / 04 / 10$
\end{tabular}

METHODS/Discussion: Carbon $\left(\delta^{13} \mathrm{C}\right)$ and nitrogen $\left(\delta^{15} \mathrm{~N}\right)$ analyses were performed on freeze dried, ground samples using a ThermoFinnigan Delta $\mathrm{V}$ plus stable isotope mass spectrometer coupled to a Thermo Flash elemental analyzer. Internal laboratory standards composed of marine chitin (Fisher Scientific) and calibrated to NIST standards were employed to correct sample data to international reference scales. Typical sample sizes analyzed were 0.5 to $1.0 \mathrm{mg}$. Typical precision of repeated chitin internal standards was $0.1 \%$ for $\mathrm{C}$ and $0.2 \%$ for $\mathrm{N}(1 \mathrm{SD})$. Values are given vs. $\mathrm{vPDB}(\mathrm{C})$ and air $(\mathrm{N})$ standard scales.

Sample isotopic values are plotted in Figure 1 along with the 2007 biota data. Most samples fall along a group with a trend of co-varying $\mathrm{C}$ and $\mathrm{N}$ values. Literature values for isotopic changes between trophic levels predict a shift of $+0-1 \%$ for $\mathrm{C}$ and $2-4 \%$ for N (Fry 1991; Hansson et al. 1997; Fantle et al. 1999; Benstead et al. 2006). Several conclusions can be drawn from this dataset. First, most organisms appear to be using a marine planktonic food source of around -20 to -22 per mil in C and 6-8 in N. Both these numbers are reasonable, the $\mathrm{C}$ is the average isotopic value for phytoplankton (Fry 1996) and the $\mathrm{N}$ value matches well with values of 5-6 measured for marine nitrate isotopes in the region (Brandes 1997). If the assumption is made that this is the food source, then the mussels are generally in the first trophic level, while the other biota samples are in the second or third trophic level. Basically anything from about 11-13 in $\delta^{15} \mathrm{~N}$ is in the second level and $14-16 \delta^{15} \mathrm{~N}$ is in the third. There is a lot of overlap suggesting that several organisms are mixotrophs. There are two noticeable outlier groups. The benthic primary producer seagrass isotopic values are enriched in ${ }^{13} \mathrm{C}$ and depleted in ${ }^{15} \mathrm{~N}$. This value is distinct enough in carbon isotopic value to strongly influence consumers utilizing this food source. Most of the samples do not appear to reflect such utilization with the exception of the sample points 16-18 (Shiner Perch from Vendovi). 


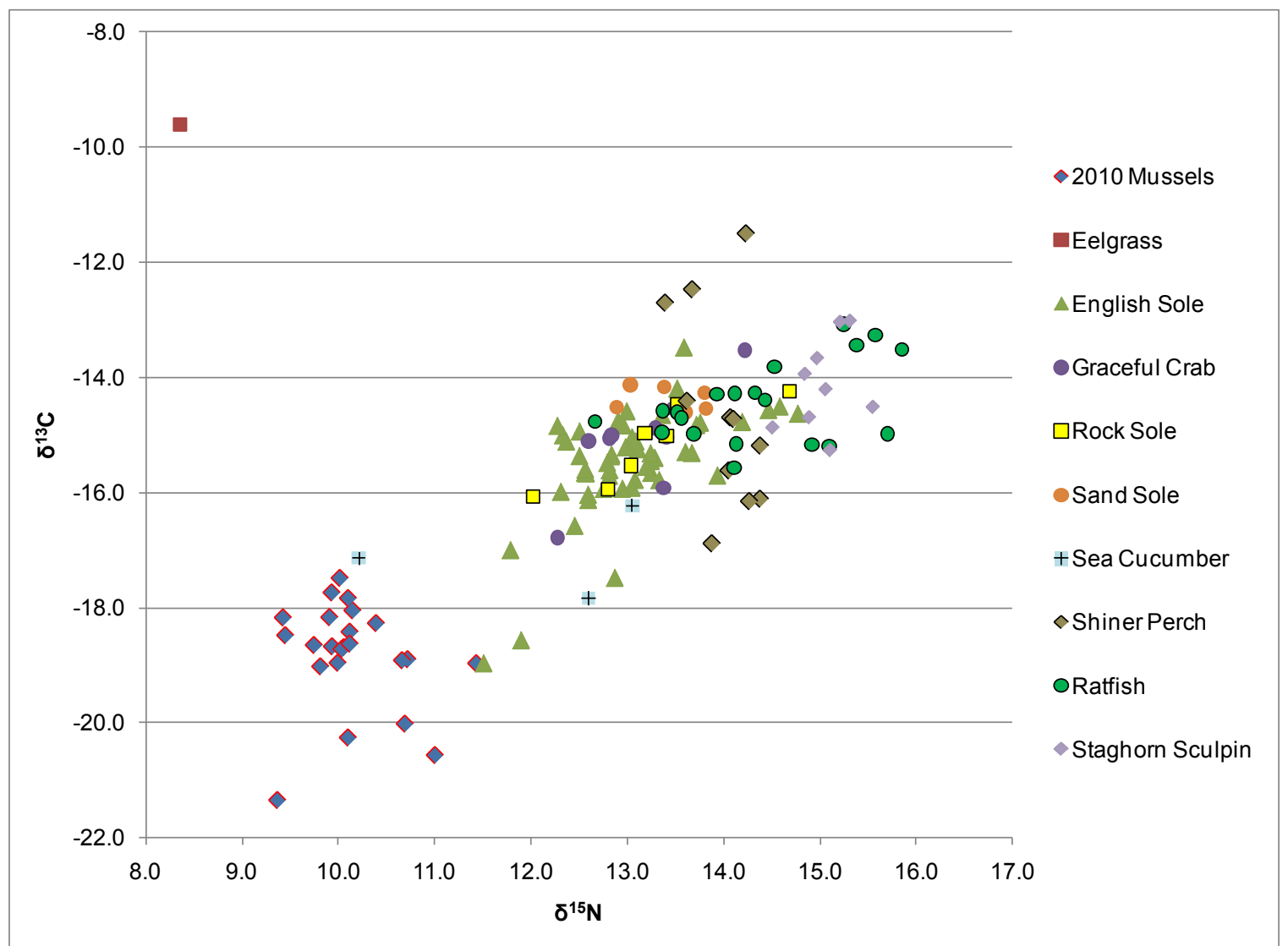

Figure 1. Stable carbon and nitrogen isotope ratios for the 2010 indigenous mussels compared to the PSAMP 2007 biota data. 


\section{Sample Custody Records}

- Field Collection Worksheets

- \&RP SRMLAN

- Sample Login 
Page: 1 , of, COC Number: $\operatorname{coc} 20100108$

Project No.: 43043

Project Name: TMDL in Sinclair \& Dyes Inlets

Project Manager: Jill Brandenberger

Phone: (360) 681-3668
SAMPLE CHAIN OF CUSTODY FORM

Date: $1 / 8 / 2010$

\section{Battelle}

Marine Sciences Laboratory

1529 West Sequim Bay Road

Sequim, Washington 98382

Laboratory: Battelle MSL

Address: $1529 \mathrm{~W}$. Sequim Bay Road

Sequim, WA 98382

sampling cocations: manchester lab pier (MCP(ER), Sinclair Iulet waterman $P t$ (SIWP) o Rosf Point ( SIRP)

\section{Attention: Jill Brandenberger \\ Observations, Instructions}

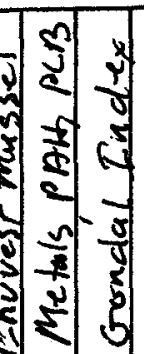

Date/Time Matrid

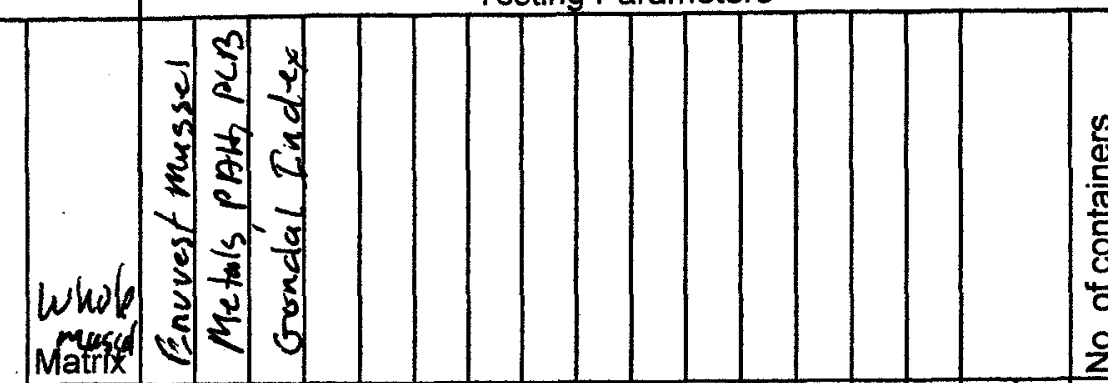

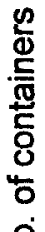

Lab.

Lab ID

\section{Sample ID}

1181101513 Musal

MWIOENOL-2 1530

MU COENOL -

MW10 EN 02-1

MW $10 \mathrm{ENO2-2}$

MWIOENO2-3

Mw10 ENO2-1

MWL10 ENO3-2

Murcu ENOG

Mu 10 GNOS-1

Mw10 EN/O5-?

MN 10 ENOS-3

MWLO EN O6-1

mw10 GNO6-2

MWLOEN $06-3$

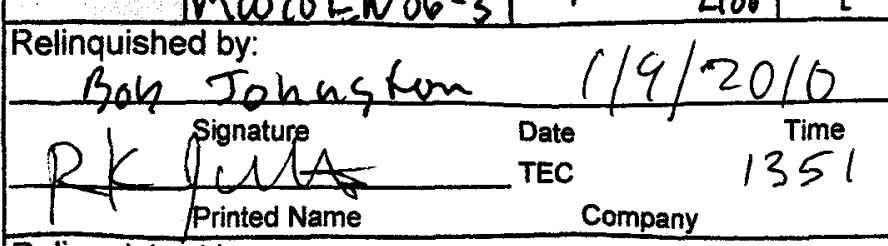

Relinquished by

1545

1513

1530

1545

1715

\begin{tabular}{l|l|}
$x$ & $x$ \\
\hline & $x$
\end{tabular}

之 StationID

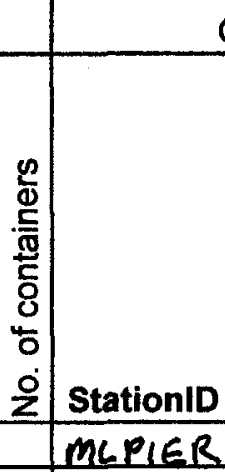

$1 \quad \begin{array}{cc}\text { Manchester lab Pier } 40 \\ 11\end{array}$

1715

1900

3.020

$x$

\begin{tabular}{|c|c|}
\hline & ce Al GOSTS \\
\hline & \#2LAISTI \\
\hline & $134 / S T$ \\
\hline SIWP & watermanPt. $\# 1$ \\
\hline 7 & waterman Pt $\# 2$ \\
\hline DRP & $\begin{array}{l}\text { Waterman }+ \text { Composite } \\
\text { Sindar Inlet Rosst: }\end{array}$ \\
\hline SLRP & Sinclair Inlet Ross Pf +13 \\
\hline & $61+2$ \\
\hline & " thsto 4 \\
\hline & Histo 42 \\
\hline & thisto 43 \\
\hline
\end{tabular}

$+x$

Received by: $\quad$ Total \# of Containers

Shipment Method: Grombe Truzsfer by

Shipment Method: Cary

Sample Disposition:

Distribution:

Received by:

Signature

Date

Time

Signature

Date

Time

1) 2 copies to the Laboratory

2) 1 copy to project manager

3) Return completed original to

Ambient vionitoring, Sinclair-Dyesonlets ${ }^{2010}$ Regional Muss etWatch, Chemistry Lata Repor
Printed Name

Company

Battelle Marine Sciencesagaberpatory 
SAMPLE CHAIN OF CUSTODY FORM

Date: $H / 2 / 2010$

Page: 1 of 3

$\operatorname{COC}$ Number 20100112A

Project No.: 43043

Project Name: ENWEST Ambient Monitoring Mussel Watch Stations

Project Manager: Jill Brandenberger

Phone: (360) 681-3668

Lab. Use
only:

Lab ID

Sample ID $\quad \begin{aligned} & \text { Collection } \\ & \text { Date/Time }\end{aligned}$

Date/Time

20100/12 MUSO/=1 1112/100909

$20100122 \mathrm{musb1}-2$

$20100112 \mathrm{mus} 61-3$

20 bol12musoz-1

20100112 musol.2

20100112 musoz-3

muso3-1

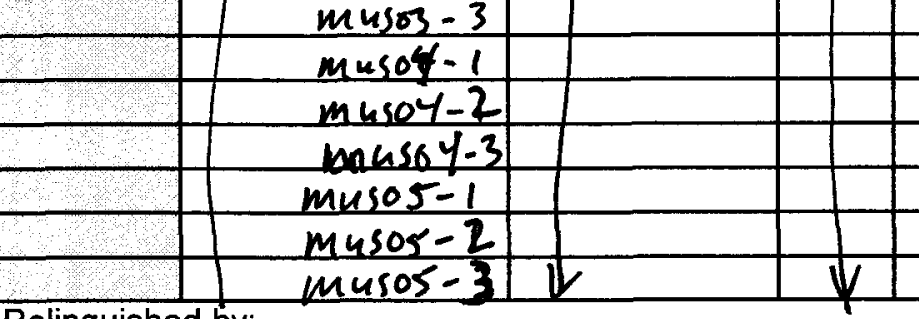

\section{Relinquished by:}

-

R Signature

BK Junster $1 / 3 / 2010$ Printed Name

Relinquished by:

Date
TEC
Company

Time

Company

$$
\text { Signature }
$$

Date

Printed Name

Company
EVENT: Mussel watch Samplin?

Sinclair d Dyes Intets

Testing Parameters

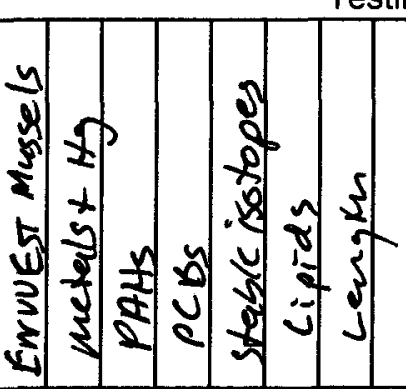

\section{Battelle}

Marine Sciences Laboratory 1529 West Sequim Bay Road Sequim, Washington 98382

Laboratory: Battelle MSL

Address: 1529 W. Sequim Bay Road

Sequim, WA 98382

\section{Attention: Jill Brandenberger}

Observations, Instructions

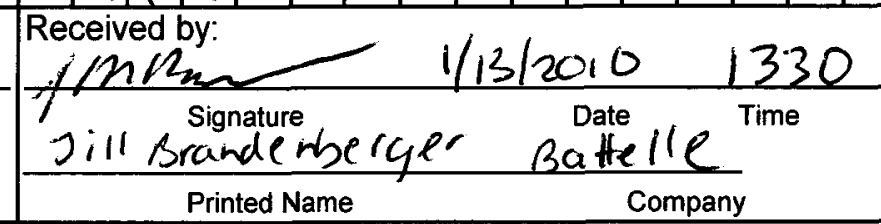

Received by:

$-$

Signature

Printed Name

Date

Company \begin{tabular}{l|c} 
StationID & Comments \\
\hline PSO4-1 & Rs Charlic Piver Ened
\end{tabular} PSO4-2 Charlie pirer sw end PSOY-3 charloe pres s side PSO3-1 near of DSNSO1S PSO3-2 . PSNO15 pso3-3 " PSNOO15 PSO6-1 006 E end on Quay are P506-2 006 neer of 19 p506-3 ope arer $6 \% 19$ PSO 8-1 DDS off Rerge pS08-2 DDS off Bare pso9-3 DDS off Bary psog-1 psoq- 2023 no4 of 18 Psog-5 D04 of 18

Total \# of Containers Shipment Method: Shipment Method:

Sample Disposition:

Distribution:

1) 2 copies to the Laboratory

2) 1 copy to project manager

3) Return completed original to

Battelle Marine Sciences Laboratory 


\section{SAMPLE CHAIN OF CUSTODY FORM}

Date: $1 / / 12 / 2010$

Page: $\frac{11 / 2}{2}$ of 3

COC Numberoc $2 0 \longdiv { 1 0 0 1 / 2 B }$

Project No:: 43043

Project Name: ENWEST Ambient Monitoring Mussel Watch Stations

Project Manager: Jill Brandenberger

Phone: (360) 681-3668

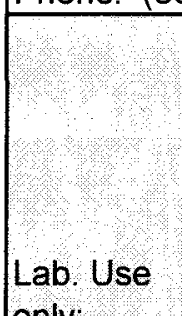

only:

Lab ID

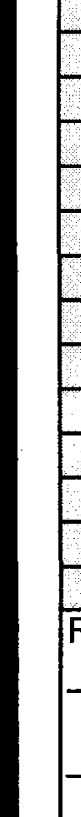

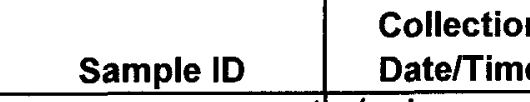

Testing Parameters

\section{Battelle}

Marine Sciences Laboratory 1529 West Sequim Bay Road Sequim, Washington 98382 Laboratory: Battelle MSL

Address: 1529 W. Sequim Bay Road Sequim, WA 98382

\section{Attention: Jill Brandenberger}

Observations, Instructions

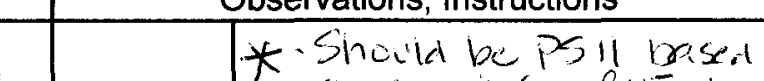

\section{Company} Prin

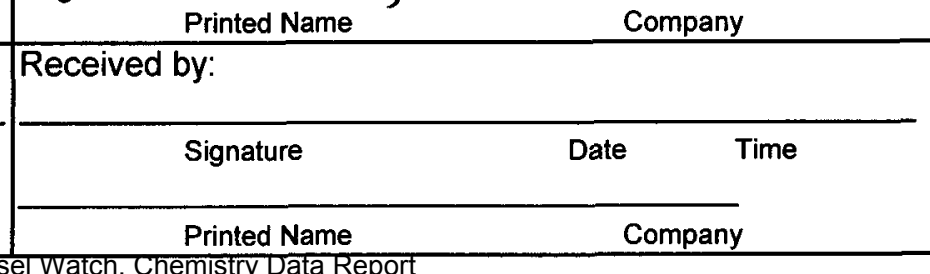

\section{Sisim-1 Sinclair Marina}

sisim-2 11

sisim-3 i.

sipom-1 Port Orchevel Marina

sipom-2 " "

sipom-3 "ll "

POP ISP-1 Aortorchard Pose T/lakee State Park POPISP-2 Illanee State Park PopISP-3 i. $u$ u POPTPD-1 Illahee Port Pock PBPIPD-2 tilahee Port Doek PoPIPD-3 + llahee Port Dock Total \# of Containers

Shipment Method: Shipment Method:

Sample Disposition: Distribution:

1) 2 copies to the Laboratory

2) 1 copy to project manager

3) Return completed original to Battelle Marine Sciences Laboratory 


\section{SAMPLE CHAIN OF CUSTODY FORM}

Date: $11 / 212010$

Page: $\frac{3}{3}$ of 3 coc Numbec $0.20100112 C$

Project No:: 43043

Project Name: ENVVST Ambient Monitoring Mussel Watch Stations Project Manager: Jill Brandenberger

Phone: (360) 681-3668

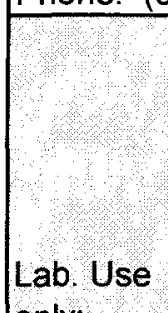

only:

Lab ID
Collection

Sample ID

\section{Sample ID}

Date/Time

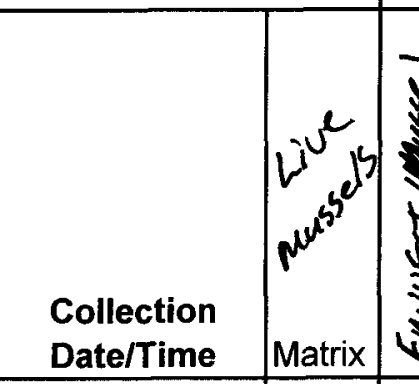

Testing Parameters

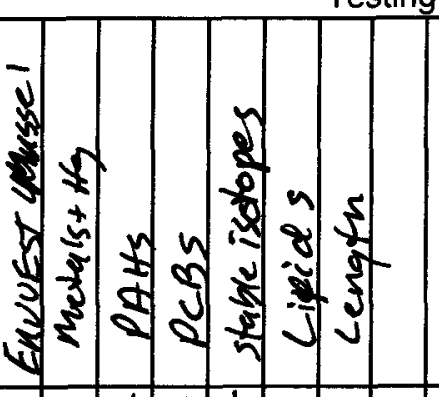

\section{Battelle}

Marine Sciences Laboratory 1529 West Sequim Bay Road Sequim, Washington 98382

Laboratory: Battelle MSL

Address: 1529 W. Sequim Bay Road Sequim, WA 98382

\section{Attention: Jill Brandenberger}

Observations, Instructions

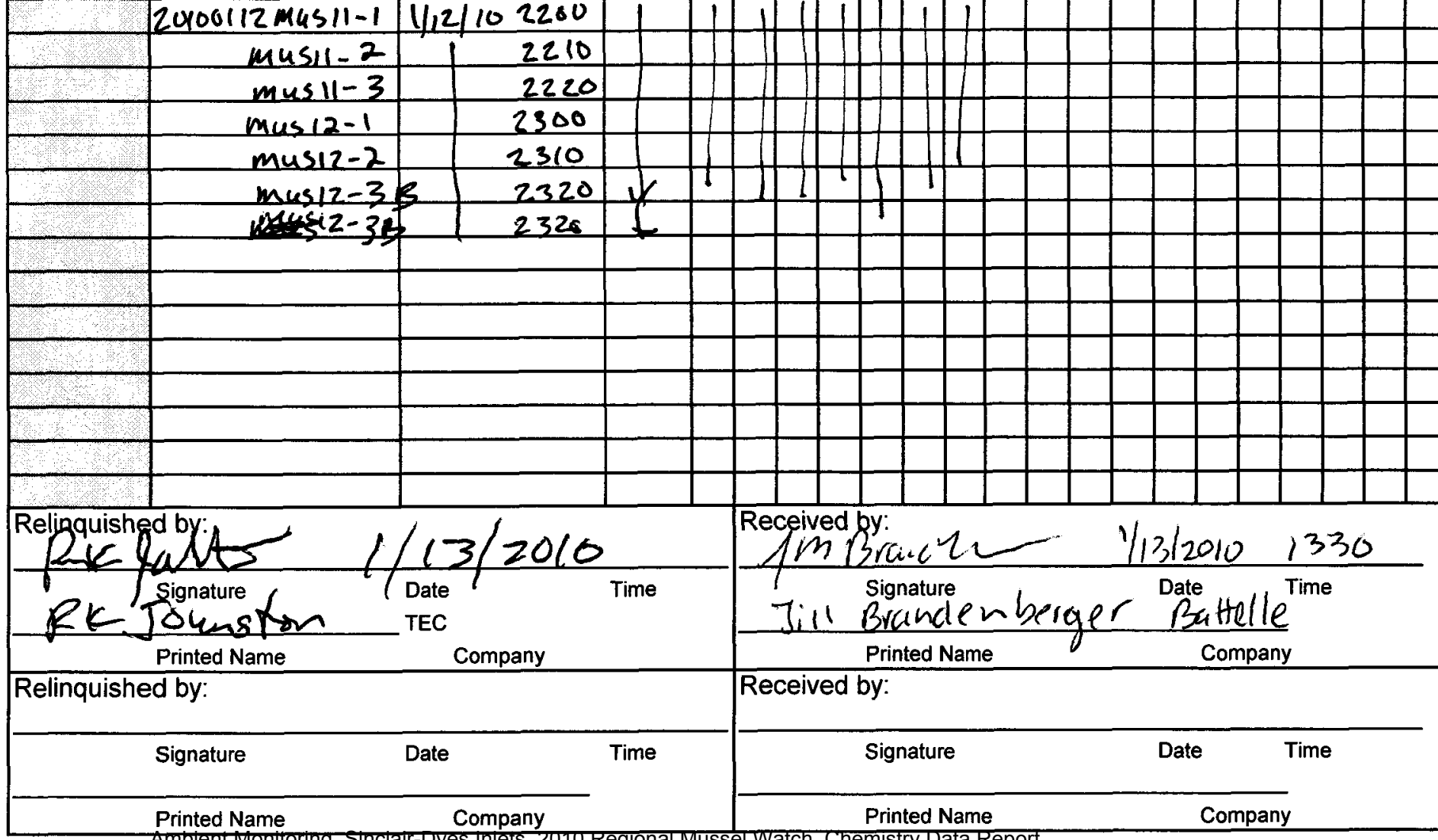

\section{StationID Comments} PWN LP -1 Por + Wash Narroute Lions Pork PuNLP-2 Lious Park PLNLP-3 Lians Park DYOTS-1 Dyes Inlet old Town Siluedsk DY075-2 old town siverdale Dyors $-3 / 47$ old Town siluedale DYoT-3 3$\}$ Snowe snarte

\section{Total \# of Containers}

Shipment Method:

Shipment Method:

Sample Disposition:

\section{Distribution:}

1) 2 copies to the Laboratory

2) 1 copy to project manager

3) Return completed original to

Battelle Marine Sciences Laboratory 
SAMPLE CHAIN OF CUSTODY FORM

Date: $\frac{2 / 2 / 2010}{7}$ of 7 COC Number:

Project No:

Project Name: ENVEST Ambient Monitoring

Project Manager:

Phone:

$$
\text { Pho }
$$

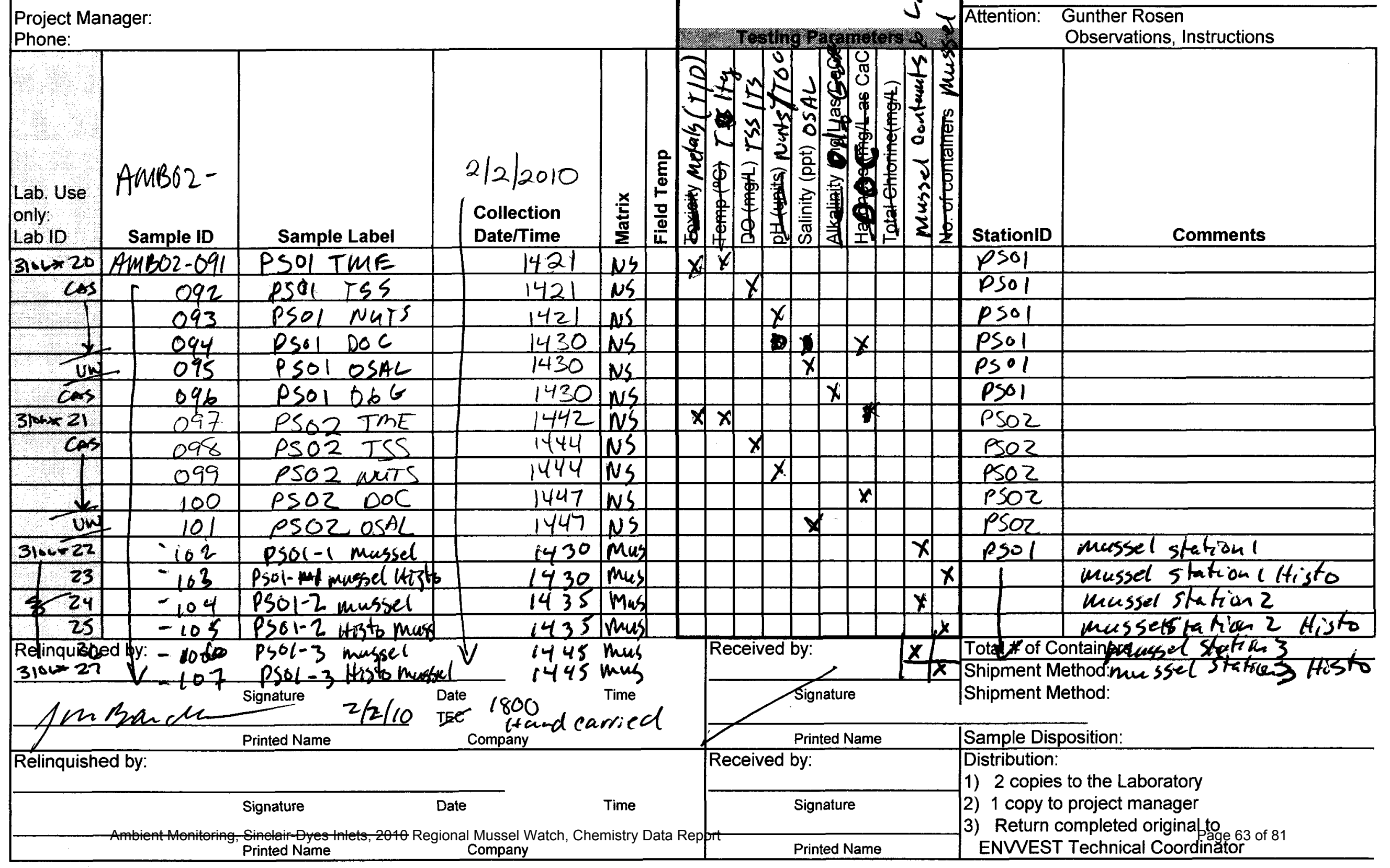




\section{SAMPLE CHAIN OF CUSTODY FORM}

Date: $\frac{213 / 10}{1 / 2} \quad 1002$ for MUSSELS

COC Number: ${ }^{\circ}-\quad$ An 512011

Project No.:

Project Name: ENWEST Ambient Monitoring

Mussel Watch Stations

Project Manager: Jill Brandenberger

Phone: (360) 681-3668

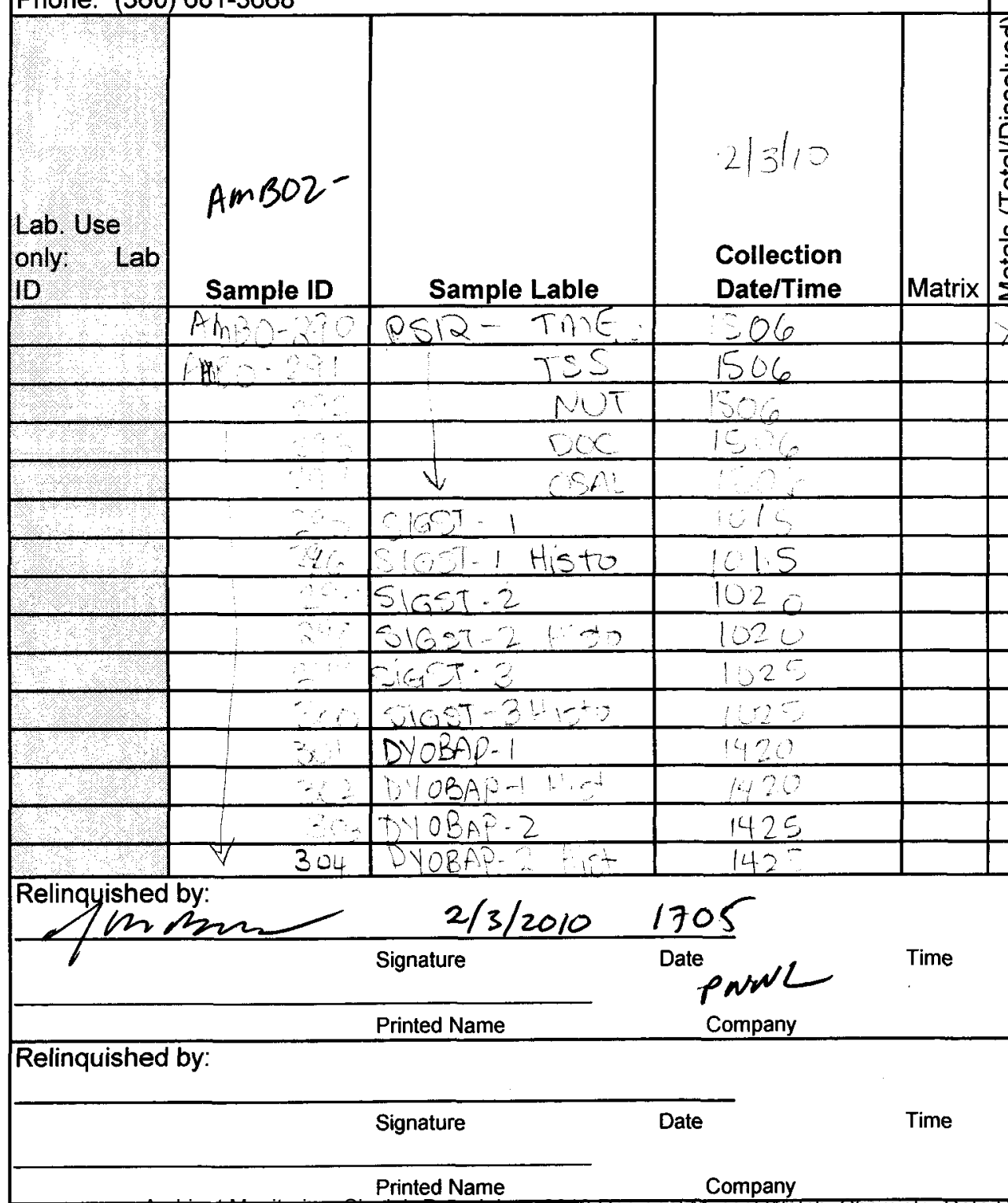

EVENT:

\section{Battelle}

Marine Sciences Laboratory 1529 West Sequim Bay Road Sequim, Washington 98382

Laboratory: Battelle MSL

Address: $\quad 1529$ W. Sequim Bay Road

Sequim, WA 98382

Attention: Jill Brandenberger Observations, Instructions

$\begin{array}{cc}\text { Attention: } & \begin{array}{l}\text { Jill Brandenberger } \\ \text { Observations, Instructions }\end{array}\end{array}$

Testing Parameters

\begin{tabular}{c|} 
Received by: \\
\hline Signature
\end{tabular}

Total \# of Containers

Shipment Method:

Shipment Method:

Sample Disposition:

Distribution:

1) 2 copies to the Laboratory

2) 1 copy to project manager

3) Return completed original to

Battelle Marine Sciences Laborator 
SAMPLE CHAIN OF CUSTODY FORM

\section{Date:}

Page: COC Number: of 8 Project No.

Project Name: ENVVEST Ambient Monitoring Mussel Watch Stations

Project Manager: Jill Brandenberger Phone: (360) 681-3668

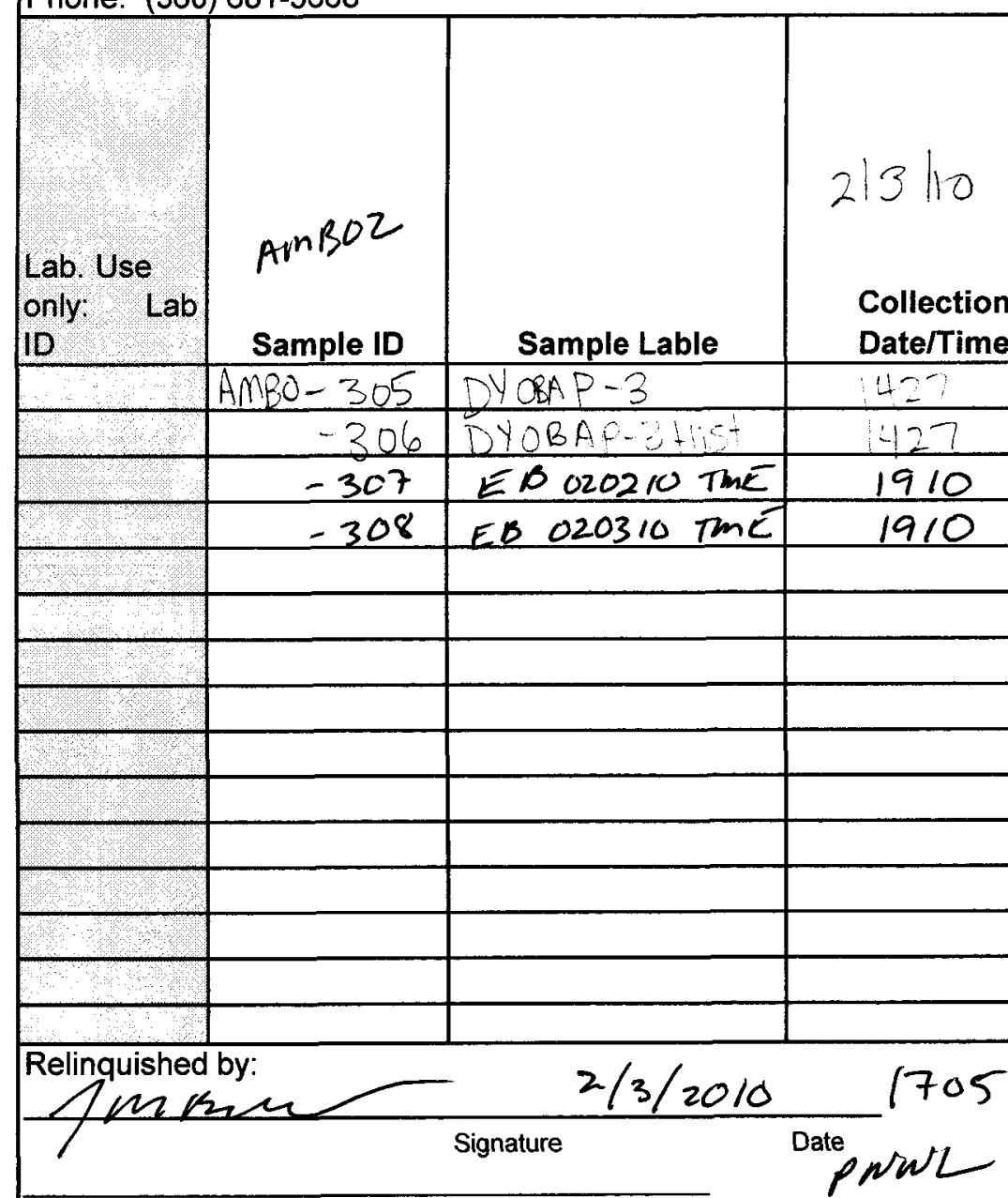

Printed Name

Company

\section{Relinquished by:}

Signature

Date

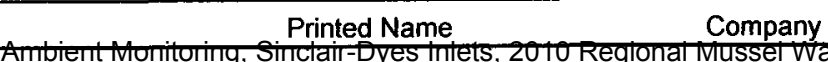

\section{Battelle}

Marine Sciences Laboratory 1529 West Sequim Bay Road Sequim, Washington 98382

Laboratory: Battelle MSL

Address: $\quad 1529$ W. Sequim Bay Road

Sequim, WA 98382

Attention: Jill Brandenberger Observations, Instructions

\begin{tabular}{cc} 
Attention: & $\begin{array}{r}\text { Jill Brandenberger } \\
\text { Observations, Instructions }\end{array}$ \\
\hline
\end{tabular}

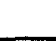

DYOBAP

Comments

$\downarrow$

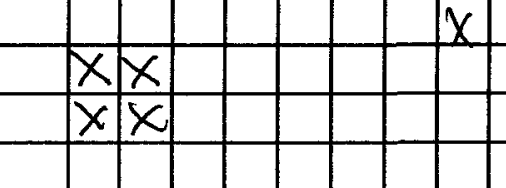

Time

Time
Received by

\begin{tabular}{|c|}
\hline Signature \\
\hline Printed Name \\
\hline Received by: \\
\hline Signature \\
\hline
\end{tabular}

Total \# of Containers

Shipment Method:

Shipment Method:

Sample Disposition:

Distribution:

1) 2 copies to the Laboratory

2) 1 copy to project manager

3) Return completed original to

Battelle Marine Sciences Laboratory 
SAMPLE CHAIN OF CUSTODY FORM

Date: $2 / 4 / 2010$

Page: 1 of 3

COC Number:

Project No:

Project Name: ENWEST Ambient Monitoring

Mussel Watch Stations

Project Manager: Jill Brandenberger

Phone: (360) 681-3668

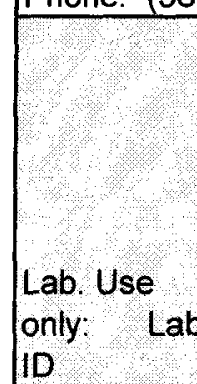

b

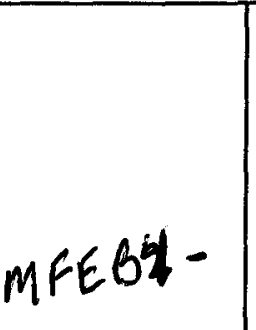

\begin{tabular}{l|l}
\hline & $M+E B$ \\
\hline & \\
\hline &
\end{tabular}

-606 POPBWN-31tisto

- OOZ KPTPIER-1

- 008 KPTPIER-1 Histo

-809 KPTPLER-2

- O10 KPTRIER-2HISA

- 11 KPTPIER-3

- 012 KPTPIER-3itisk

-013 KPTLAG-1

-014 KPTLAGtitisto \begin{tabular}{c|c|c}
-014 & KPTLAGHATS & 142 \\
-015 & KPTLAG-2 & 1420
\end{tabular}

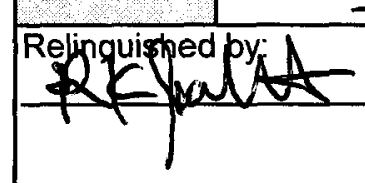

$2 / 5 / 2040$

Signature

21412010

Collection

Date/Time

1106

1606

420

1120

1141

$4|4|$

301

1301

1310

$13 / 0$

1320

1320

$141^{2}$

Relinquished by:

Company

Signature

Printed Name

Time

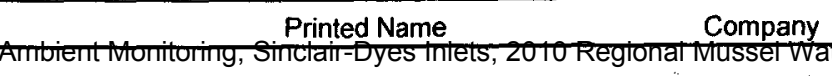

\section{EVENT: \\ Mussel sampling \\ Ni Port orcherd Passage}

Testing Parameters

\begin{tabular}{ll} 
Attention: & $\begin{array}{l}\text { Jill Brandenberger } \\
\text { Observations, Instructions }\end{array}$ \\
\hline
\end{tabular}

Address: 1529 W. Sequim Bay Road

\section{Battelle}

Marine Sciences Laboratory

1529 West Sequim Bay Road

Sequim, Washington 98382

Laboratory: Battelle MSL

Sequim, WA 98382

Observations, Instructions 
SAMPLE CHAIN OF CUSTODY FORM

Date: $2 / 4 / 2010$

COC Number:

Project No.

Project Name: ENWEST Ambient Monitoring

Mussel Watch Stations

Project Manager: Jill Brandenberger

Phone: (360) 681-3668

\begin{tabular}{|c|c|c|c|c|}
\hline $\begin{array}{l}\text { Lab. Use } \\
\text { only: Lab } \\
\text { ID }\end{array}$ & $\begin{array}{l}\text { MVIFG4 } \\
\text { Sample ID }\end{array}$ & Sample Lable & $\begin{array}{l}2 / 4 / 20 / 0 \\
\text { Collection } \\
\text { Date/Time }\end{array}$ & ${ }_{\text {Matrix }}^{2}$ \\
\hline & -016 & EPTLAG-2Histo & 1420 & musod \\
\hline & -017 & KPTLAL -3 & 1450 & \\
\hline & -018 & KPTLAG-3iti3to & 1450 & \\
\hline & -019 & $A P H G \sqrt{3}-1$ & 1626 & \\
\hline & -020 & $A P$ itCh-1Histo & 1626 & \\
\hline & -611 & $A P H C B-2$ & 1700 & \\
\hline & -022 & $A P+C B-2 h i 3 h$ & 1700 & \\
\hline & -023 & AP KIANA-1 & 1900 & \\
\hline & -024 & APLIANA - Iftrsto & 1800 & \\
\hline & -025 & APKIANA - 2 & 1405 & \\
\hline & -026 & APKIANA-2HBH & 1805 & \\
\hline & -027 & APKIANA-3 & 1620 & \\
\hline & -028 & AOKIANA - SHEN & 1820 & \\
\hline & -029 & $\angle B P M S L-1$ & 2020 & \\
\hline & -030 & $\angle B P M S C-A+N$ & 2070 & \\
\hline \multirow{2}{*}{\multicolumn{5}{|c|}{ Relinquishegby: $2 / 5 / 2600$}} \\
\hline & & & & \\
\hline & & Printed Name & Company & \\
\hline \multicolumn{5}{|c|}{ Relinquished by: } \\
\hline & & Signature & ate & Time \\
\hline & & $P$ & & \\
\hline
\end{tabular}

EVENT:

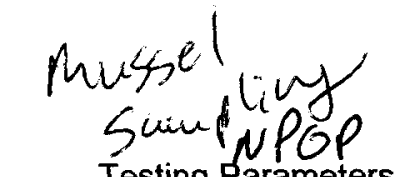

Testing Parameters

\section{Battelle}

Marine Sciences Laboratory

1529 West Sequim Bay Road Sequim, Washington 98382

Laboratory: Battelle MSL

Address: 1529 W. Sequim Bay Road

Sequim, WA 98382

Attention: Jill Brandenberger

Observations, Instructions

Observations, Instructions

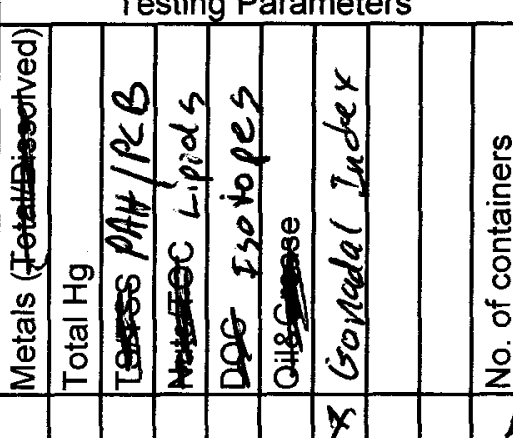

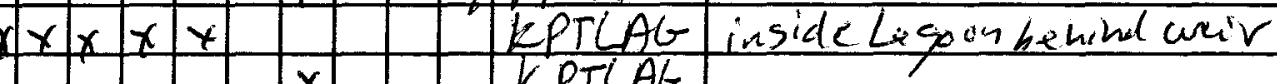

$x \times x \times x=\frac{A P H C B}{\text { in Big Rock }}$

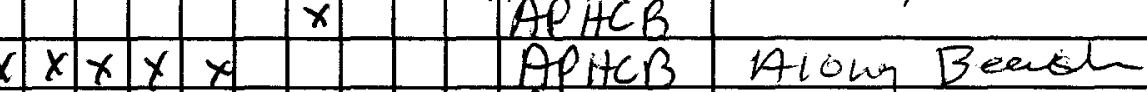

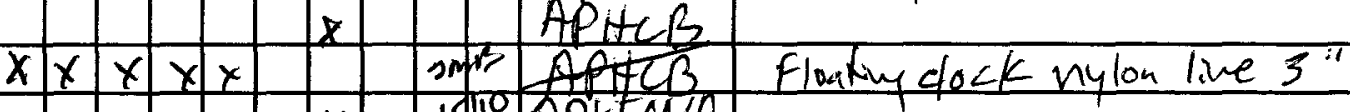

$x \times \times \times$ X $x$ 2/5

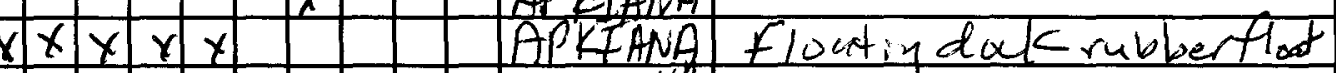

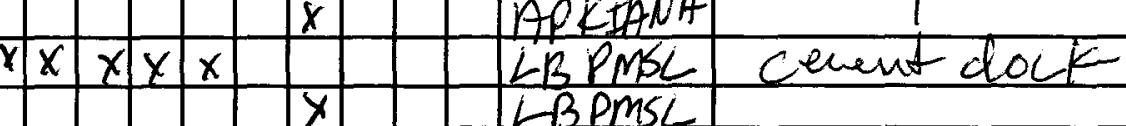

Received by: $\quad 2 / 5$ Total \# of Containers

Jindice 2/5/2010 Shipment Method:

Signature PNNL Shipment Method:

\begin{tabular}{|c|}
\hline Printed Name \\
\hline Received by: \\
\hline Signature \\
\hline
\end{tabular}

Sample Disposition:

Distribution:

1) 2 copies to the Laboratory

2) 1 copy to project manager

3) Return completed original to

Printed Name

Battelle Marine Sciences Laboratory 
SAMPLE CHAIN OF CUSTODY FORM

Date: $2 / 4 / 2070$

Page:

COC Number:

3

Project No:

Project Name: ENVVEST Ambient Monitoring

Mussel Watch Stations

Project Manager: Jill Brandenberger

Phone: (360) 681-3668

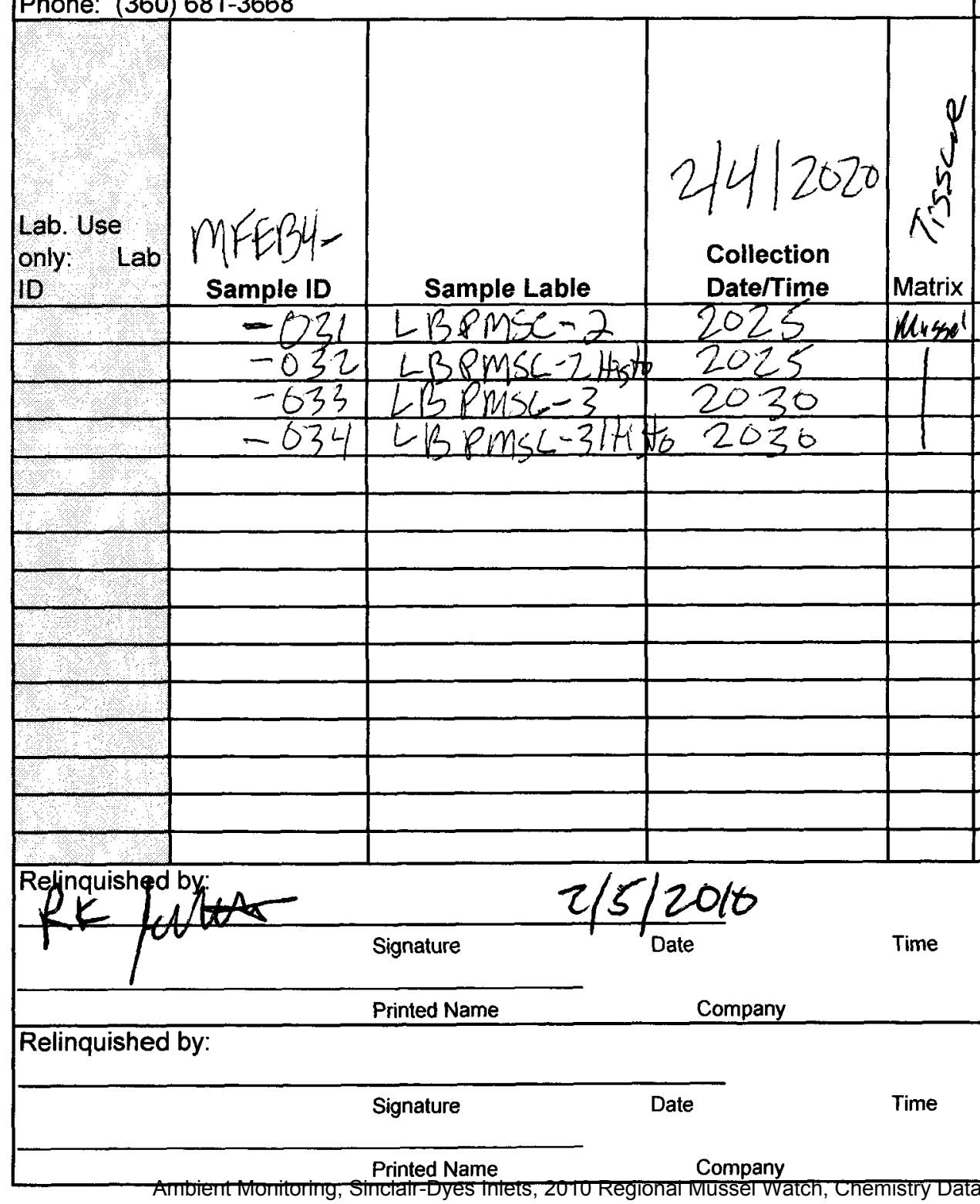

\section{Battelle}

Marine Sciences Laboratory

1529 West Sequim Bay Road

Sequim, Washington 98382

Laboratory: Battelle MSL

Address: 1529 W. Sequim Bay Road

Sequim, WA 98382

EVENT: NPOP

Testing Parameters

Attention: Jill Brandenberger

Observations, Instructions
Received by:
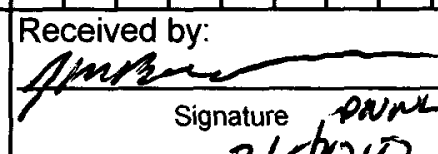

$2 / 5,2010$

$\frac{\text { Received by: }}{\text { Signature }}$
Total \# of Containers

Shipment Method:

Shipment Method:

Sample Disposition:

Distribution:

1) 2 copies to the Laboratory

2) 1 copy to project manager

3) Return completed original to

Battelle Marine Sciences Laboratory
Comments cenent doct 
MUSSEL WATCH PROGRAM DATA SHEET

Site: Puget Ht Sound Naval Shipyard PSOI site Code: PSOL

Date: $2 / 2 / 2010$

Time Arrive: $/ Y \mathrm{Cl}$

Time Leave: 1455

Latitude: 47,55385 Longitude: 122,65785

Weather: shear w/ Sum

Mussel Collectors: Bob Johnston

Data Recorder: Johnston

SITE WATER PARAMETERS

Water Temperature $\left({ }^{\circ} \mathrm{C}\right): 8,7 \quad$ conditivy $50,027 \mathrm{mseimeus} / \mathrm{cm}$

pH 7.62: Turn 2,9, DO $8.41 \mathrm{mg} / \mathrm{c}$

Tidal Station: Breneivfon, sinclair Infect, port orchard

Time of Low Tide: 1337

Height of Low Tide: $2.8 \quad \mathrm{ft} . \square \mathrm{m}$.

STATION DESCRIPTIONS

Latitude: Longitude: Start Time: 1430

Station Description: of of elechieal cable hinging in water

Top 6-12ft depth

Substrate: electrical cable upper end Height of Collection: ft. - - $m$.

Highest Distribution of Mussels (compared to water level at time of collection):

Species Collected: my li ins spp.

Latitude: Longitude: Start Time: 1435

Station Description: off of electrical cable hanging in water $12-18 \mathrm{ft}$ depth

Substrate: electrical cable lower end Height of Collection: $\mathrm{ft}-\mathrm{tzm}$

Highest Distribution of Mussels (compared to water level at time of collection): $-1-6$

Species Collected: Mytilus sf'

Latitude:

Longitude:

Start Time: 1445

Station Description: Ga beach next to sampling location

Substrate: rock

Height of Collection:

ft. 4 . $\mathrm{m}$.

Highest Distribution of Mussels (compared to water level at time of collection): \pm

Species Collected: Mytilus spf.

Ambient Monitoring, Sinclair-Dyes Inlets, 2010 Regional Mussel Watch, Chemistry Data Report

Version 3.1MESO -2010
Page 69 of 81 
Check Boxes for Site Conditions:

\begin{tabular}{|l|l|l|}
\hline \multicolumn{1}{|c|}{ Condition } & PSOL & Description 2/2/2010 \\
\hline Creosote & $n_{0}$ \\
\hline oil on water & no \\
\hline oil on beach & no \\
\hline Garbage & \\
\hline & \\
\hline & \\
\hline & & \\
\hline
\end{tabular}

Observations and General Notes (i.e. interesting or unusual conditions, information, comments, etc):

In west end of Shipyard in corner between carrier (Independence) and charleston Beach directly near where callow Ave out fall disknerges. mussels were collected from electiculal cable hanging in the water of of the bow if carrier - list sample was from $6^{\prime}-12^{c}$ of submerged cable 6 sencond sample was $12^{\prime}-18^{\prime}+t$ if submerged cable. 3 sample cane from beach on rocks just above cow tide

Ambient Monitoring, Sinclair-Dyes Inlets, 2010 Regional Mussel Watch, Chemistry Data Report

Page 70 of 81 


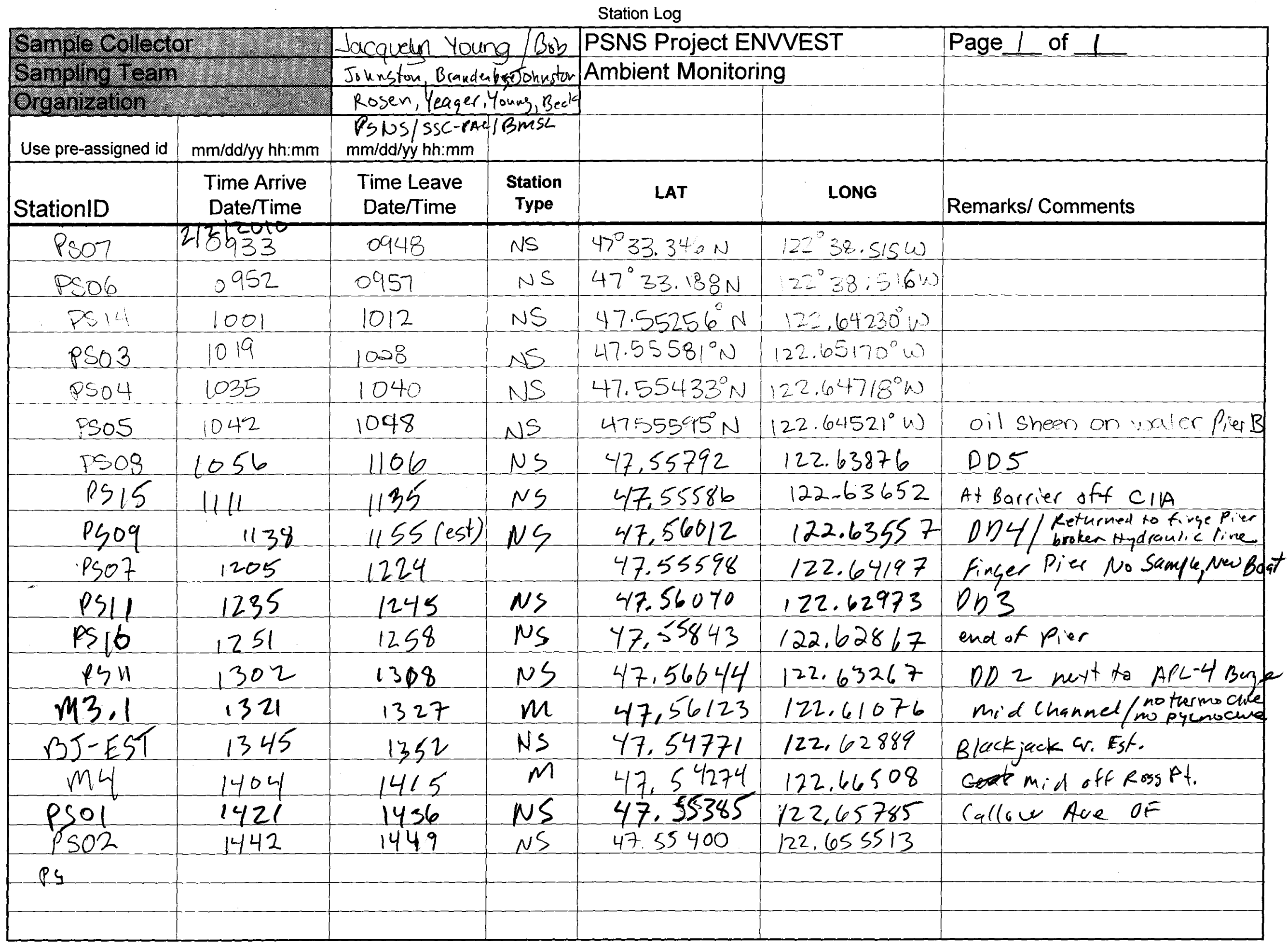

Page 71 fie nt
Station Type: Marine (M), Nearshore (NS), Stream (S), Stormwater (SW), Outfall (OF), Mussel (Mussel), Sediment (Std); Other - specify 
Central File \#: 3106 Sample No(s): / -

Batch:

Project Name:

Project Manager:

TO BE COWLLETED BY PROJECT MANAGER (PHior to errival when possible)

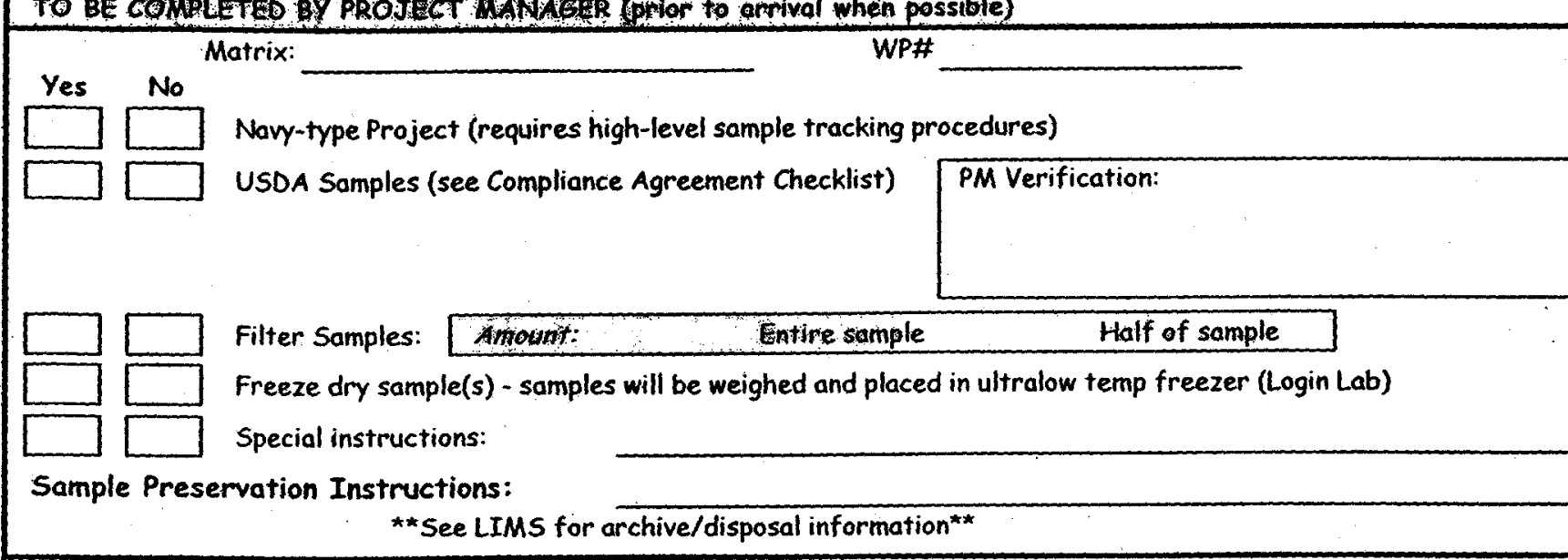

\section{TO BE COMPLETED UPON SAMPLE ARRIVALROG-IN}

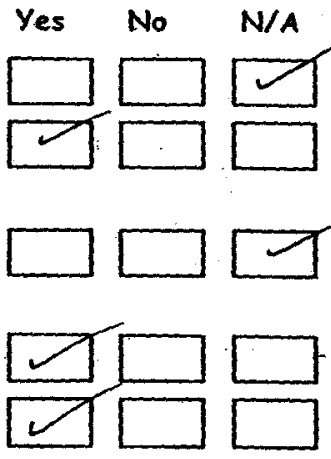

Sample condition(s):

Container type:
Seal intact? YES NO Custody seal present

Cooler temperature (acceptable range: $4 \pm 2^{\circ} \mathrm{C}$ or solids:frozen) (if multiple coolers, note temp. of each)
$\# 13.5$ \#2.8 $\begin{array}{r}43 \quad 4.7 \\ \# 4 \quad 5.2 \\ \hline{ }^{\circ} \mathrm{C}\end{array}$ Comment/Remedy:

Were all chain of custody forms signed and dated?

Were samples filtered at MSL?

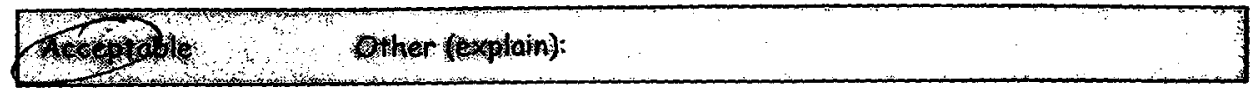

Notes:

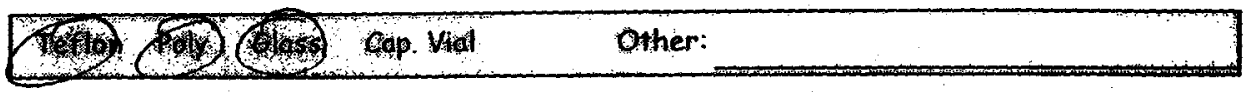$$
\text { Completed By: } 1 \mathrm{~m} \mathrm{M2s}
$$

\section{SAMPLE PRESERVATION}

$\square$ Sample(s) were preserved prior to árrival at MSL (noted on COC / Sample / per PM Instruction)

$\square$ Random pH checked for $\sim 10 \%$ of samples (use dip paper) Sample IDs:

$\square$ Complete $\mathrm{pH}$ check required for project (use $\mathrm{pH}$ meter and record on pH Record form)

$\square$ Sample(s) were preserved at MSL

Type:

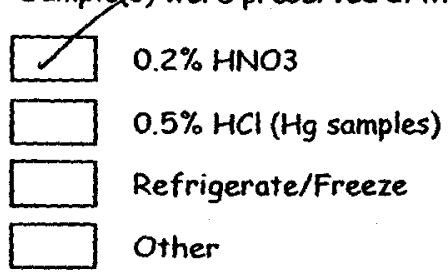

Date/Time: $2 / 2 / 10 \quad 1910$

Completed By:

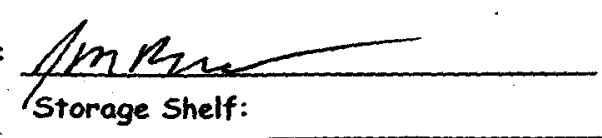

Notes: $\frac{1209050<\text { Lot\# }}{\text { Lotes: }}$

Notes:

Notes: 


\section{SAMPLE CHAIN OF CUSTODY FORM}

Date:

Page: $4 / 5 / 0$

1 of 2

COMPOSITES

Project Name:

ENUET Amb Mon.

Chemistry Task Manager: Jill Brandenberger

Phone Number: $\quad 360-681-4564$

\section{Sample ID $\quad \begin{aligned} & \text { Collection } \\ & \text { Date }\end{aligned}$}

20100112 Mus 1 ifomp NOTE:
JUST USE
not is en
ermis
112110 20100112 mis o2-comp

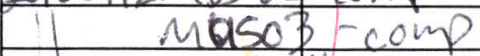

muso4-comp

Muso6-lm

maso7-6mp

musogtcomp

Musto-comp

Mus 11 tom

Musiz-lomp

202

MW1O ENOI- $00 \mathrm{mp}(1 / 3 / 10)-203), \square$

Relinquished by:

\begin{tabular}{clc}
\hline Signature & Date & Tim \\
\hline Printed Name & Company & \\
\hline
\end{tabular}

Relinquished by:

Printed Name

Time

Signature

Date

Time

Printed Name
Ambient Monitoring, Sine

\section{Battelle}

Marine Sciences Laboratory

1529 W. Sequim Bay Road

Sequim, Washington 98382

Laboratory:

Address:

Attention:

Testing Parameters

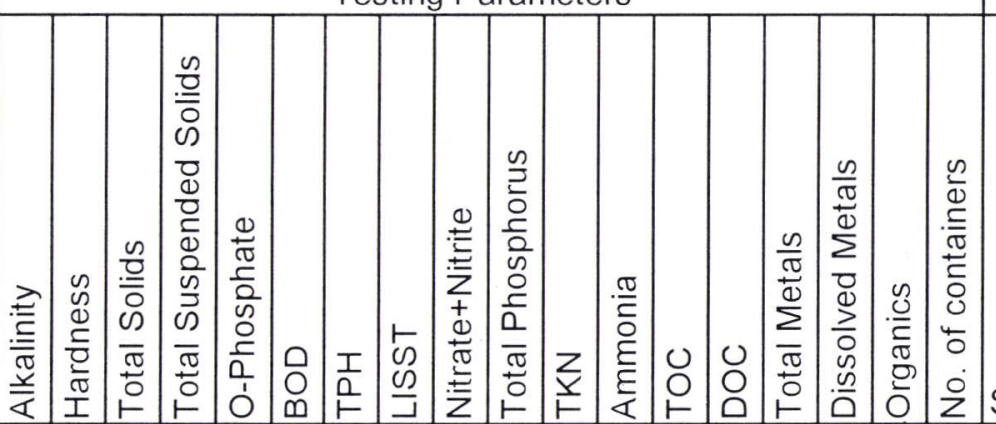

Observations, Instructions

* Should be psir based

on email trom RKJ atd

5/20110 coum 5/2010

Station ID

PSO4-comp3106 215. \begin{tabular}{|c|c|}
\hline $503-\operatorname{com} 0$ & 216 \\
\hline $1506-\operatorname{com} p$ & 217
\end{tabular} PSO - comp 218 Psoa-comp 219. PS10- comp 220 जा IM- comp y 221 , \begin{tabular}{l} 
POPISP-com \\
\hline $1 P O M-\operatorname{com}$
\end{tabular} POPIPDPUNLP-comp 225. 1) 10 TS-comp 226. MLPIER - COMP3106.227

Total \# of Containers $=0$ Shipment Method:

Special Requirements or Conditions:

Sample Disposition: Distribution:

1) 2 copies to the Laboratory

2) 1 copy to project manager

3) Return completed original to Battelle Marine Sciences Laboratory 


\section{SAMPLE CHAIN OF CUSTODY FORM}

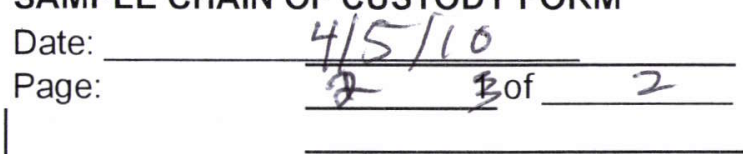

Project Name: ENVEST Amb.MON

Chemistry Task Manager: Jill Brandenberger

Phone Number: $\quad 360-681-4564$

Sample ID

MWOEN

Date

MWIO EN OF comp

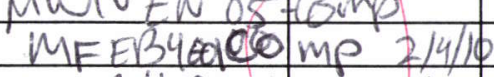

MFEB $4007-60$ mp

MFEB4013-domp

MFEBH-019-comp

MFEBU-023-con

MFEB4-029-comp 3106 .

\begin{tabular}{|c|c|c|c|}
\hline 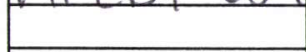 & 1 & \multicolumn{2}{|l|}{212} \\
\hline & & 213 & \\
\hline & & 214 & \\
\hline & & & \\
\hline & & & \\
\hline & & & \\
\hline
\end{tabular}

Relinquished by:

\begin{tabular}{|llll}
\hline & Signature & Date & Time \\
\hline & Printed Name & Company & \\
\hline Relinquished by: & & & \\
\hline & Signature & Date & Time
\end{tabular}

Printed Name
Ambient Montoring, Smeta

\section{Battelle}

Marine Sciences Laboratory 1529 W. Sequim Bay Road

Sequim, Washington 98382

$$
\text { Mussel wateh Samplng Laboratory:Battelle MSI }
$$

Testing Parameters

Attention:

Observations, Instructions

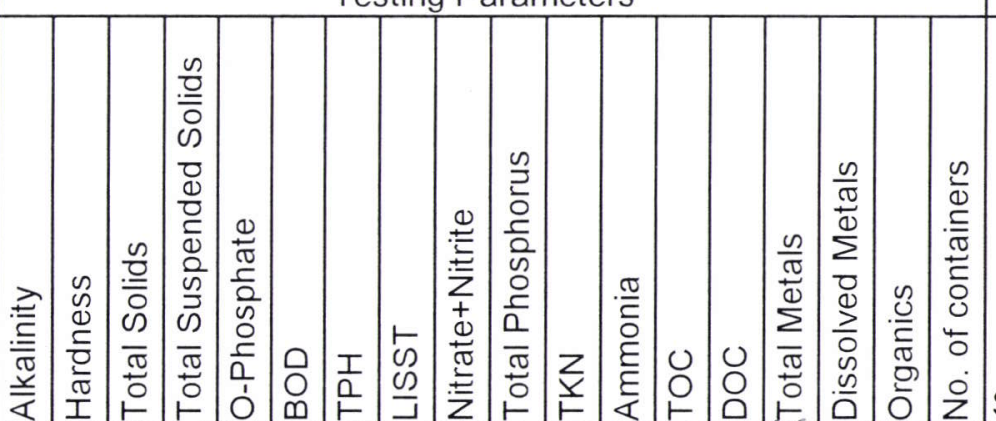

Station ID

Slup-comp $3106 \cdot 228$ \begin{tabular}{|l|r|}
\hline SIRP-comp & 229 \\
\hline POPBWN-comp & 230 \\
\hline
\end{tabular} KPTPIER-COMP 23, KP TLAG-Cop Wh 232 $A P H C B-\operatorname{com} D$ $x \quad$ APK FANA TOmP $x \quad x \quad$ DOBAD-comp 236. $x \quad x \quad S I G S T-c o m p$ $x$ pso 1-comp 2106.238 * $x$ LB

Total \# of Containers $=0$ Shipment Method:

Special Requirements or Conditions:

Sample Disposition: Distribution:

1) 2 copies to the Laboratory

2) 1 copy to project manager

3) Return completed original to Battelle Marine Scjences Laboratory 


\section{SAMPLE LOGIN}

Project Manager: Brandenberger

Date Received: $4 / 7 / 2010$

Batch: 6

Login Designee: McGahan

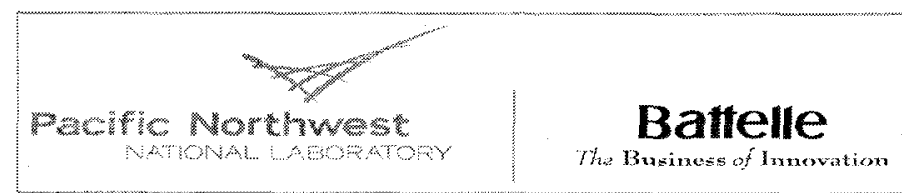

Marine Sciences Laboratory 1529 West Sequim Bay Road Sequim, Washington 98382 $\mathrm{PH}:(360) 681-4565$

Project: ENVVEST Ambient Monitoring, March 2010

\begin{tabular}{|c|c|c|c|c|c|c|c|}
\hline Sponsor ID & Station ID & Description & Battelle Code & Matrix & Storage Location & $\begin{array}{l}\text { Requested } \\
\text { Parameters } \\
\end{array}$ & $\begin{array}{c}\text { Collection } \\
\text { Date } \\
\end{array}$ \\
\hline 20100112MUS01-C & PS04 & PSNS NAVSTA Mid (CP) & $3106-191$ & mussel Comp & Outside Freezer & Organics & $01 / 12 / 10$ \\
\hline 20100112MUS02-C & PS03 & PSNS NAVSTA West (DP) & $3106-192$ & mussel Comp & Outside Freezer & Organics & $01 / 12 / 10$ \\
\hline 20100112MUS03-C & PS06 & PSNS CIA West (6) & $3106-193$ & mussel Comp & Outside Freezer & Organics & $01 / 12 / 10$ \\
\hline 20100112MUS04-C & PS08 & PSNS CIA MidE (5) & $3106-194$ & mussel Comp & Outside Freezer & Organics & $01 / 12 / 10$ \\
\hline 20100112MUS05-C & PS09 & PSNS CIA MidW (4) & $3106-195$ & mussel Comp & Outside Freezer & Organics & $01 / 12 / 10$ \\
\hline 20100112MUS06-C & PS11 & PSNS CIA East (3) & $3106-196$ & mussel Comp & Outside Freezer & Organics & $01 / 12 / 10$ \\
\hline 20100112MUS07-C & SISIM & Sinclair Inlet Sinclair Marina & $3106-197$ & mussel Comp & Outside Freezer & Organics & $01 / 12 / 10$ \\
\hline 20100112MUSO9-C & POPISP & Port Orchard Pass Illahee State Park & $3106-198$ & mussel Comp & Outside Freezer & Organics & $01 / 12 / 10$ \\
\hline 20100112MUS08-C & SIPOM & Sinclair Inlet Port Orchard Marina & $3106-199$ & mussel Comp & Outside Freezer & Organics & $01 / 12 / 10$ \\
\hline 20100112MUS10-C & POPIPD & Port Orchard Pass Illahee State Dock & $3106-200$ & mussel Comp & Outside Freezer & Organics & $01 / 12 / 10$ \\
\hline 20100112MUS11-C & PWNLP & Port Washington Narrows Lions Park & $3106-201$ & mussel Comp & Outside Freezer & Organics & $01 / 12 / 10$ \\
\hline 20100112MUS12-C & DYOTS & Dyes Inlet Old Town Silverdale & $3106-202$ & mussel Comp & Outside Freezer & Organics & $01 / 12 / 10$ \\
\hline MW10EN01-C & MLPIER & Manchester Lab Pier & $3106-203$ & mussel Comp & Outside Freezer & Organics & $01 / 08 / 10$ \\
\hline MW10EN03-C & SIWP & Sinclair Inlet Waterman Point & $3106-204$ & mussel Comp & Outside Freezer & Organics & $01 / 08 / 10$ \\
\hline MW10EN06-C & SIRP & Sinclair Inlet Ross Point & $3106-205$ & mussel Comp & Outside Freezer & Organics & $01 / 08 / 10$ \\
\hline MFEB4-001C & POPBWN & Port Orchard Passage Brownsville & $3106-206$ & mussel Comp & Outside Freezer & Organics & $02 / 04 / 10$ \\
\hline MFEB4-007C & KPTPIER & Keyport NUWC Pier & $3106-207$ & mussel Comp & Outside Freezer & Organics & $02 / 04 / 10$ \\
\hline MFEB4-013C & KPTLAG & Keyport Lagoon & $3106-208$ & mussel Comp & Outside Freezer & Organics & $02 / 04 / 10$ \\
\hline MFEB4-019C & APHCB & Agate Pass BI Hidden Cove Beach & $3106-209$ & mussel Comp & Outside Freezer & Organics & $02 / 04 / 10$ \\
\hline MFEB4-023C & APKIANA & Agate Pass Kiana Lodge & $3106-210$ & mussel Comp & Outside Freezer & Organics & $02 / 04 / 10$ \\
\hline
\end{tabular}




\section{SAMPLE LOGIN}

Project Manager: Brandenberger

Date Received: 4/7/2010

Batch: 6

Login Designee: McGahan

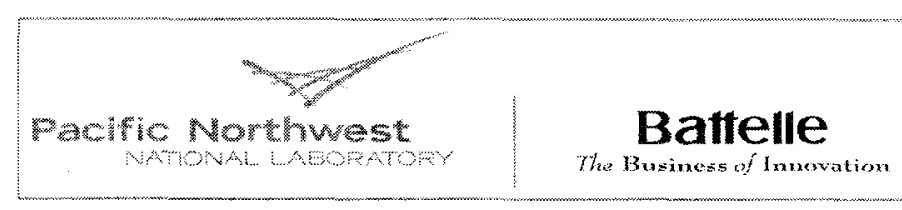

Marine Sciences Laboratory 1529 West Sequim Bay Road Sequim, Washington 98382 PH: (360) 681-4565

Project: ENVVEST Ambient Monitoring, March 2010

\begin{tabular}{|c|c|c|c|c|c|c|c|}
\hline Sponsor ID & Station ID & Description & Battelle Code & Matrix & Storage Location & $\begin{array}{l}\text { Requested } \\
\text { Parameters }\end{array}$ & $\begin{array}{c}\text { Collection } \\
\text { Date }\end{array}$ \\
\hline MFEB4-029C & LBPMSC & $\begin{array}{l}\text { Liberty Bay Poulsbo Marina Science } \\
\text { Center }\end{array}$ & $3106-211$ & mussel Comp & Outside Freezer & Organics & $02 / 04 / 10$ \\
\hline AMB02-301C & DYOBAP & Dyes Inlet Ostrich Bay Ammo Pier & $3106-212$ & mussel Comp & Outside Freezer & Organics & $02 / 03 / 10$ \\
\hline AMB02-295C & SIGST & Sinclair Inlet head at Gorst. & $3106-213$ & mussel Comp & Outside Freezer & Organics & $02 / 03 / 10$ \\
\hline AMB02-102C & PS01 & PNSN Inactive Fleet Callow Ave OF & $3106-214$ & mussel Comp & Outside Freezer & Organics & $02 / 02 / 10$ \\
\hline
\end{tabular}




\section{SAMPLE LOGIN}

Project Manager: Brandenberger

Date Received: $\quad 4 / 7 / 2010$

Batch: 6

Login Designee: McGahan

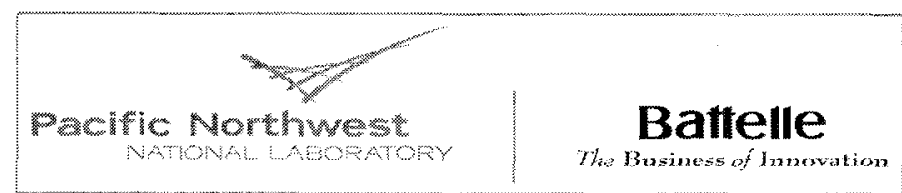

Marine Sciences Laboratory 1529 West Sequim Bay Road Sequim, Washington 98382 $\mathrm{PH}:(360)$ 681-4565

Project: ENVVEST Ambient Monitoring, March 2010

\begin{tabular}{|c|c|c|c|c|c|c|c|}
\hline Sponsor ID & Station ID & Description & Battelle Code & Matrix & Storage Location & $\begin{array}{l}\text { Requested } \\
\text { Parameters }\end{array}$ & $\begin{array}{c}\text { Collection } \\
\text { Date }\end{array}$ \\
\hline 20100112MUS01-C & PS04 & PSNS NAVSTA Mid (CP) & $3106-215$ & mussel Comp & Deep Freezer B-2 & metals & $01 / 12 / 10$ \\
\hline 20100112MUS02-C & PS03 & PSNS NAVSTA West (DP) & $3106-216$ & mussel Comp & Deep Freezer B-2 & metals & $01 / 12 / 10$ \\
\hline 20100112MUS03-C & PS06 & PSNS CIA West (6) & $3106-217$ & mussel Comp & Deep Freezer B-2 & metals & $01 / 12 / 10$ \\
\hline 20100112MUS04-C & PS08 & PSNS CIA MidE (5) & $3106-218$ & mussel Comp & Deep Freezer B-2 & metals & $01 / 12 / 10$ \\
\hline 20100112MUS05-C & PS09 & PSNS CIA MidW (4) & $3106-219$ & mussel Comp & Deep Freezer B-2 & metals & $01 / 12 / 10$ \\
\hline 20100112MUS06-C & PS 11 & PSNS CIA East (3) & $3106-220$ & mussel Comp & Deep Freezer B-2 & metals & $01 / 12 / 10$ \\
\hline 20100112MUS07-C & SISIM & Sinclair Inlet Sinclair Marina & $3106-221$ & mussel Comp & Deep Freezer B-2 & metals & $01 / 12 / 10$ \\
\hline 20100112MUS09-C & POPISP & Port Orchard Pass Illahee State Park & $3106-222$ & mussel Comp & Deep Freezer B-2 & metals & $01 / 12 / 10$ \\
\hline 20100112MUS08-C & SIPOM & Sinclair Inlet Port Orchard Marina & $3106-223$ & mussel Comp & Deep Freezer B-2 & metals & $01 / 12 / 10$ \\
\hline 20100112MUS10-C & POPIPD & Port Orchard Pass Illahee State Dock & $3106-224$ & mussel Comp & Deep Freezer B-2 & metals & $01 / 12 / 10$ \\
\hline 20100112MUS11-C & PWNLP & Port Washington Narrows Lions Park & $3106-225$ & mussel Comp & Deep Freezer B-2 & metals & $01 / 12 / 10$ \\
\hline 20100112MUS12-C & DYOTS & Dyes Inlet Old Town Silverdale & $3106-226$ & mussel Comp & Deep Freezer B-2 & metals & $01 / 12 / 10$ \\
\hline MW10EN01-C & MLPIER & Manchester Lab Pier & $3106-227$ & mussel Comp & Deep Freezer B-2 & metals & $01 / 08 / 10$ \\
\hline MW10EN03-C & SIWP & Sinclair Inlet Waterman Point & $3106-228$ & mussel Comp & Deep Freezer B-2 & metals & $01 / 08 / 10$ \\
\hline MW10EN06-C & SIRP & Sinclair Inlet Ross Point & $3106-229$ & mussel Comp & Deep Freezer B-2 & metals & $01 / 08 / 10$ \\
\hline MFEB4-001C & POPBWN & Port Orchard Passage Brownsville & $3106-230$ & mussel Comp & Deep Freezer B-2 & metals & $02 / 04 / 10$ \\
\hline MFEB4-007C & KPTPIER & Keyport NUWC Pier & $3106-231$ & mussel Comp & Deep Freezer B-2 & metals & $02 / 04 / 10$ \\
\hline MFEB4-013C & KPTLAG & Keyport Lagoon & $3106-232$ & mussel Comp & Deep Freezer B-2 & metals & $02 / 04 / 10$ \\
\hline MFEB4-019C & APHCB & Agate Pass BI Hidden Cove Beach & $3106-233$ & mussel Comp & Deep Freezer B-2 & metals & $02 / 04 / 10$ \\
\hline MFEB4-023C & APKIANA & Agate Pass Kiana Lodge & $3106-234$ & mussel Comp & Deep Freezer B-2 & metals & $02 / 04 / 10$ \\
\hline
\end{tabular}




\section{SAMPLE LOGIN}

Project Manager: Brandenberger

Date Received: 4/7/2010

Batch: 6

Login Designee: McGahan

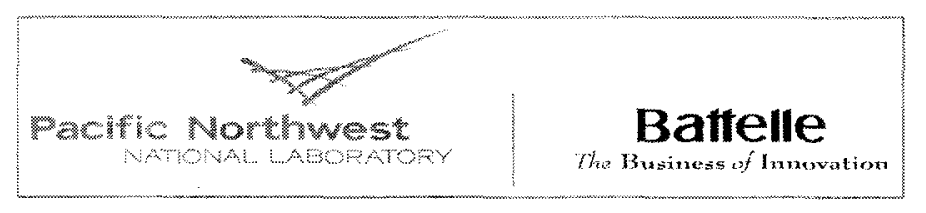

Marine Sciences Laboratory 1529 West Sequim Bay Road Sequim, Washington 98382 PH: (360) 681-4565

Project: ENVVEST Ambient Monitoring, March 2010

\begin{tabular}{|c|c|c|c|c|c|c|c|}
\hline Sponsor ID & Station ID & Description & Battelle Code & Matrix & Storage Location & $\begin{array}{l}\text { Requested } \\
\text { Parameters }\end{array}$ & $\begin{array}{l}\text { Collection } \\
\text { Date }\end{array}$ \\
\hline MFEB4-029C & LBPMSC & $\begin{array}{l}\text { Liberty Bay Poulsbo Marina Science } \\
\text { Center }\end{array}$ & $3106-235$ & mussel Comp & Deep Freezer B-2 & metals & $02 / 04 / 10$ \\
\hline AMB02-301C & DYOBAP & Dyes Inlet Ostrich Bay Ammo Pier & $3106-236$ & mussel Comp & Deep Freezer B-2 & metals & $02 / 03 / 10$ \\
\hline $\begin{array}{l}\text { AMB02-295C } \\
\text { AMB02-102C }\end{array}$ & $\begin{array}{l}\text { SIGST } \\
\text { PS01 }\end{array}$ & $\begin{array}{c}\text { Sinclair Inlet head at Gorst } \\
\text { PNSN Inactive Fleet Callow Ave OF }\end{array}$ & $\begin{array}{l}3106-237 \\
3106-238\end{array}$ & $\begin{array}{l}\text { mussel Comp } \\
\text { mussel Comp }\end{array}$ & $\begin{array}{l}\text { Deep Freezer B-2 } \\
\text { Deep Freezer B-2 }\end{array}$ & $\begin{array}{l}\text { metals } \\
\text { metals }\end{array}$ & $\begin{array}{l}02 / 03 / 10 \\
02 / 02 / 10\end{array}$ \\
\hline
\end{tabular}


Central File \#: $3106 \quad$ sample No(s): 19/-238 (COMPosite) Batch: 6

Project Name: Eurrest Ambient Movitories March 2010 Project Manager: Brandenberser

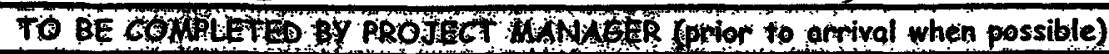

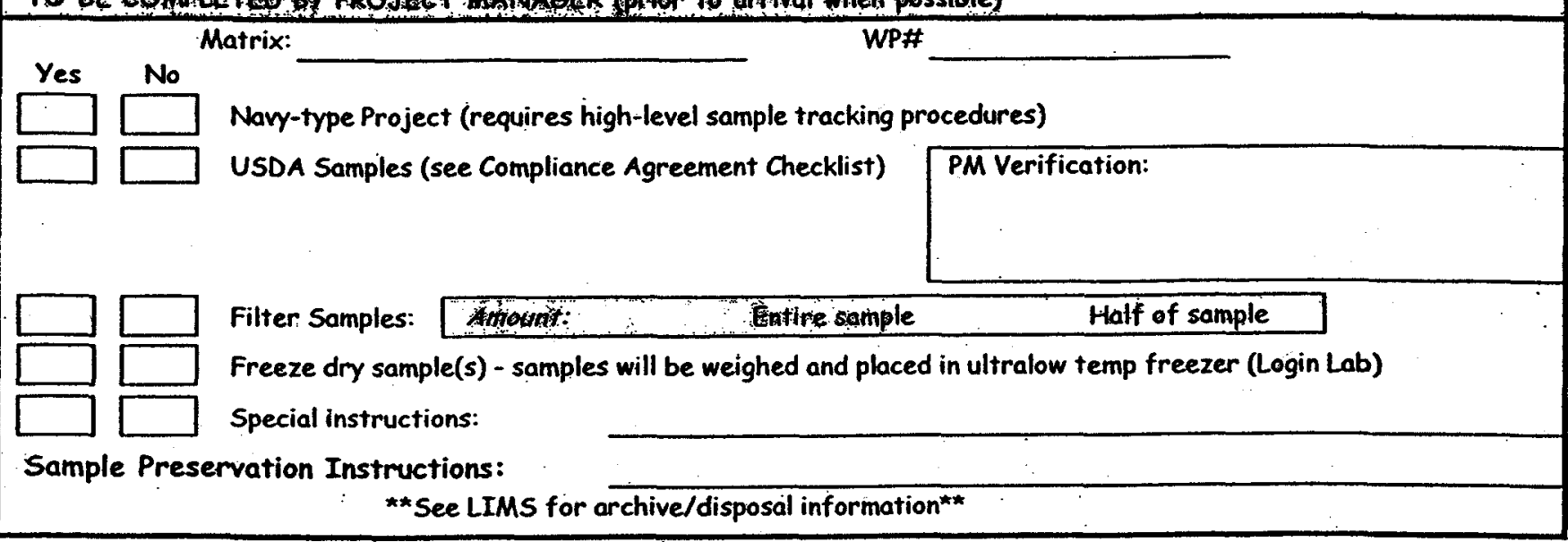

\section{TO BE COMPLETED UPON SAMPLE ARRIVALLLOG-IN}

Yes No $N / A$. Indicate in Appropriate Box

$\square$ Custody seal present Seal intact? YES NO

口 $\square$ Cooler temperature (acceptable range: $4 \pm 2^{\circ} \mathrm{C}$ or solids:frozen)

(if multiple coolers, note temp. of each)

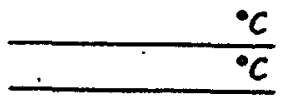

口 D Project Manager notified of any custody/login discrepancies (cooler temp. sponsor codes, etc) CommentiRemedy: Samples hand carried 's'placed cx outside frenz-

WD $\square[$ Were all chain of custody forms signed and dated?:

DEL $\square$ Were samples filtered at MSL?

Sample condition(s):

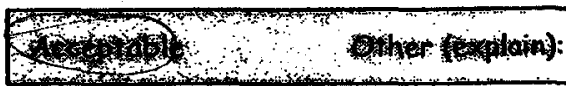

Container type:

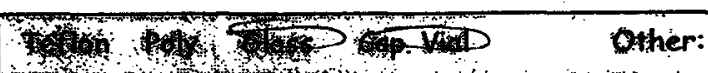

Notes:

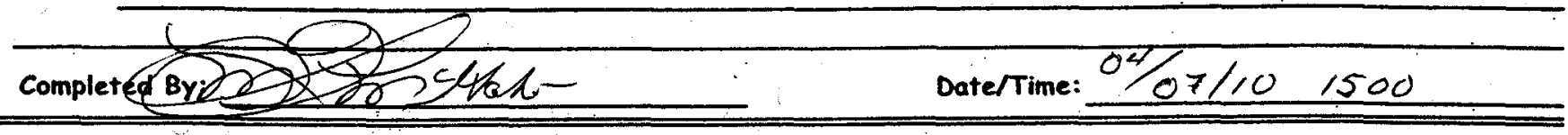

\section{SAMPLE PRESERVATION}

. Sample(s) were preserved prior to árival at MSL (noted on CoC / Sample / per PM Instruction)

$\square$ Random pH checked for $-10 \%$ of samples (use dip paper) Sample IDs:

$\square$ Complete $\mathrm{pH}$ check required for project (use $\mathrm{pH}$ meter and record on pH Record form)

Sample(s) were preserved at MSL

Type:

$0.2 \%+\mathrm{NON}$ Notes: Lot\#

$\square 0.5 \% \mathrm{HCl}$ (Hg samples)

DOJ Refrigerate/Freeze

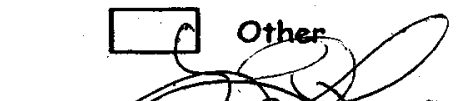

completer Bris

Storage shelf: deep keenom B-Z

Notes: Lot\#

Notes: $191-214$ outside therge $215-238$ llogekenger

Notes:

DaterTime: $04 / 07 / 10 / 545$ 


\section{References}




\section{References}

Brandenberger JM, CR Suslick, and RK Johnston. 2008. Biological Sampling and Analysis in Sinclair and Dyes Inlets, Washington: Chemical Analyses for 2007 Puget Sound Biota Study. Pacific Northwest National Laboratory, Richland, WA. Technical Report No. PNNL-17948.

Brandenberger JM, CR Suslick, and RK Johnston. 2006a. Biological Sampling and Analysis in Sinclair and Dyes Inlets, Washington: Chemical Analyses for the Caged Mussel Study. Pacific Northwest National Laboratory, Richland, WA. PNNL15835.

Brandenberger JM, CR Suslick, and RK Johnston. 2006b. Biological Sampling and Analysis in Sinclair and Dyes Inlets, Washington: Chemical Analyses for 2005 Puget Sound Biota Study. Pacific Northwest National Laboratory, Richland, WA. PNNL-15834.

Brandenberger JM, CR Suslick, and RK Johnston. 2003. 2003 Sinclair and Dyes Inlet TMDL Study: Biological Sampling and Analysis for Metals and PCBs. Pacific Northwest National Laboratory, Richland, WA.

ENVVEST (Johnston, R.K). 2006. Puget Sound Naval Shipyard \& Intermediate Maintenance Facility Project ENVVEST Community Update June 2006. Brochure and CD. Marine Environmental Support Office-NW, Space and Naval Warfare Systems Center, Bremerton, WA. August 2006. Ecology Publication Number 0610-54 http://www.ecy.wa.gov/biblio/0610054.html

Johnston, R.K., G.H. Rosen, J.M. Brandenberger, V.S. Whitney, and J.M. Wright. 2009. Sampling and Analysis Plan for Ambient Monitoring and Toxicity Testing for Sinclair and Dyes Inlets, Puget Sound, Washington. ENVVEST Planning Document.

Johnston RK, DE Leisle, JM Brandenberger, SA Steinert, M Salazar, and SM Salazar. 2007. Contaminate Residues in Demersal Fish, Invetebrates, and Deployed Mussels in Selected Areas of The Puget Sound, WA. In proceedings from 2007 Georgia Basin Puget Sound Conference, Vancouver, British Columbia, March 26-29, 2007, p. 9 pages. PNNL-SA-55152.

National Oceanic and Atmospheric Administration (NOAA) 2009. National Status \& Trends Mussel Watch Program: Mussel Watch Site Descriptions and Sampling Procedures for Washington State

SCMRC 2009. Mussel Watch Monitoring Protocols. 